\title{
Nets of standard subspaces on Lie groups
}

\author{
Karl-Hermann Neeb, Gestur Ólafsson
}

June 24, 2020

\begin{abstract}
Let $G$ be a Lie group with Lie algebra $\mathfrak{g}, h \in \mathfrak{g}$ an element for which the derivation ad $h$ defines a 3-grading of $\mathfrak{g}$ and $\tau_{G}$ an involutive automorphism of $G$ inducing on $\mathfrak{g}$ the involution $e^{\pi i \operatorname{ad} h}$. We consider antiunitary representations $(U, \mathcal{H})$ of the Lie group $G_{\tau}=G \rtimes\left\{\mathbf{1}, \tau_{G}\right\}$ for which the positive cone $C_{U}=\{x \in \mathfrak{g}:-i \partial U(x) \geq 0\}$ and $h$ span $\mathfrak{g}$. To a real subspace $\mathrm{E} \subseteq \mathcal{H}^{-\infty}$ of distribution vectors invariant under $\exp (\mathbb{R} h)$ and an open subset $\mathcal{O} \subseteq G$, we associate the real subspace $\mathrm{H}_{\mathrm{E}}(\mathcal{O}) \subseteq \mathcal{H}$, generated by the subspaces $U^{-\infty}(\varphi) \mathrm{E}$, where $\varphi \in$ $C_{c}^{\infty}(\mathcal{O}, \mathbb{R})$ is a real-valued test function on $\mathcal{O}$. Then $\mathrm{H}_{\mathrm{E}}(\mathcal{O})$ generates the complex Hilbert space $\mathcal{H}_{E}(G):=\overline{\mathrm{H}_{\mathrm{E}}(G)+i \mathrm{H}_{\mathrm{E}}(G)}$ for every non-empty open subset $\mathcal{O} \subseteq G$ (Reeh-Schlieder property).

For the real standard subspace $\mathrm{V} \subseteq \mathcal{H}$, for which $J_{\mathrm{V}}=U\left(\tau_{G}\right)$ is the modular conjugation and $\Delta_{\mathrm{V}}^{-i t / 2 \pi}=U(\exp t h)$ is the modular group, we obtain sufficient conditions to be of the form $\mathrm{H}_{\mathrm{E}}(S)$ for an open subsemigroup $S \subseteq G$. If $\mathfrak{g}$ is semisimple with simple hermitian ideals of tube type, we verify these criteria and obtain nets of cyclic subspaces $\mathrm{H}_{\mathrm{E}}(\mathcal{O}), \mathcal{O} \subseteq G$, satisfying the Bisognano-Wichmann property in a suitable sense. Our construction also yields such nets on simple Jordan space-times and compactly causal symmetric spaces of Cayley type. By second quantization, these nets lead to free quantum fields in the sense of Haag-Kastler on causal homogeneous spaces whose groups are generated by modular groups and conjugations. MSC 2010: Primary 22E45; Secondary 81R05, 81T05.
\end{abstract}

\section{Contents}

1 Introduction $\quad 2$

2 Distribution vectors of $C$-positive representations 8

2.1 The semigroup action on distribution vectors . . . . . . . . . . . 8

2.2 Real subspaces generated by distribution vectors . . . . . . . . . . . . . 13

2.3 The Reeh-Schlieder property . . . . . . . . . . . . . . . . . . 15

2.4 Support properties of corresponding distributions . . . . . . . . . . . 15

$3 C$-positive representation of 3-graded Lie groups $\quad \mathbf{1 7}$

3.1 Distribution vectors associated to standard subspaces . . . . . . . . . . 18

3.2 Extending orbit maps of distribution vectors . . . . . . . . . . . . . . 20

3.3 An example: The $a x+b$-group . . . . . . . . . . . . . . 24

4 Realizing $C$-positive representations on tubes 25

4.1 Translating between tube and ball . . . . . . . . . . . . . . 27

4.2 Unitary representations . . . . . . . . . . . . . . . . . . . . . . . . . . . . . . . . . . . . . . . . . . .

4.3 Antiunitary extensions . . . . . . . . . . . . . . . . . 31 


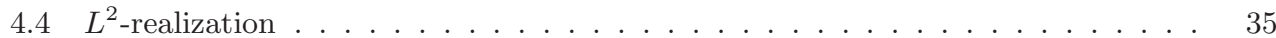

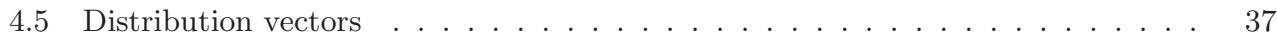

5 Nets of standard subspaces

5.1 Nets of standard subspaces on Lie groups . . . . . . . . . . . . . . . . . 39

5.2 Jordan space-times and causal symmetric spaces . . . . . . . . . . . 43

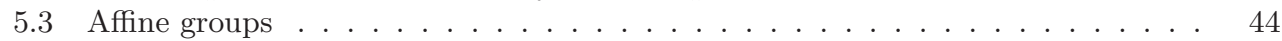

6 Perspectives 44

6.1 Extension to more general groups . . . . . . . . . . . . . . . . . . . . . . . . . . .

6.2 Relation to causal structures . . . . . . . . . . . . . . . . 45

A Distribution vectors for unitary representations 45

A.1 The topology on $\mathcal{H}^{\infty}$ and the space $\mathcal{H}^{-\infty} \ldots \ldots \ldots \ldots \ldots$

A.2 Equivariant embeddings into distributions on $G \ldots \ldots \ldots$. . . . . . 48

\section{Introduction}

Let $\mathcal{H}$ be a complex Hilbert space and $\mathcal{M} \subseteq B(\mathcal{H})$ be a von Neumann algebra. A unit vector $\Omega \in \mathcal{H}$ is called cyclic for $\mathcal{M}$ if $\mathcal{M} \Omega$ is dense in $\mathcal{H}$, and separating if the map $\mathcal{M} \rightarrow \mathcal{H}, M \mapsto M \Omega$ is injective. If $\Omega$ is both, cyclic and separating for $\mathcal{M}$, then the Tomita-Takesaki Theorem (BR87, Thm. 2.5.14]) asserts in particular that the closed real subspace

$$
\mathrm{V}:=\overline{\left\{M \Omega: M=M^{*} \in \mathcal{M}\right\}}
$$

is standard, i.e.,

$$
\mathrm{V} \cap i \mathrm{~V}=\{0\} \quad(\mathrm{V} \text { is separating) } \quad \text { and } \quad \mathcal{H}=\overline{\mathrm{V}+i \mathrm{~V}} \quad \text { ( } \mathrm{V} \text { is cyclic) }
$$

(cf. Lo08 for the basic theory of standard subspaces). To the standard subspace V, we associate a pair of modular objects $\left(\Delta_{\mathrm{V}}, J_{\mathrm{V}}\right)$ : the modular operator $\Delta_{\mathrm{V}}$ is a positive selfadjoint operator, $J_{\mathrm{V}}$ is a conjugation (an antiunitary involution), and these two operators satisfy the modular relation $J_{\mathrm{V}} \Delta_{\mathrm{V}} J_{\mathrm{V}}=\Delta_{\mathrm{V}}^{-1}$. The pair $\left(\Delta_{\mathrm{V}}, J_{\mathrm{V}}\right)$ is obtained by the polar decomposition $\sigma_{\mathrm{V}}=J_{\mathrm{V}} \Delta_{\mathrm{V}}^{1 / 2}$ of the closed operator

$$
\sigma_{\mathrm{V}}: \mathrm{V}+i \mathrm{~V} \rightarrow \mathcal{H}, \quad x+i y \mapsto x-i y
$$

with $\mathrm{V}=\operatorname{Fix}\left(\sigma_{\mathrm{V}}\right)$. The main assertion of the Tomita-Takesaki Theorem is that

$$
J_{\mathrm{V}} \mathcal{M} J_{\mathrm{V}}=\mathcal{M}^{\prime} \quad \text { and } \quad \Delta_{\mathrm{V}}^{i t} \mathcal{M} \Delta_{\mathrm{V}}^{-i t}=\mathcal{M} \quad \text { for } \quad t \in \mathbb{R} \text {. }
$$

So we obtain a one-parameter group of automorphisms of $\mathcal{M}$ (the modular group) and a symmetry between $\mathcal{M}$ and its commutant $\mathcal{M}^{\prime}$, implemented by $J_{\mathrm{v}}$.

In Algebraic Quantum Field Theory (AQFT) in the sense of Haag-Kastler, one considers nets of von Neumann algebras $\mathcal{M}(\mathcal{O}) \subseteq B(\mathcal{H})$, associated to regions $\mathcal{O}$ in some space-time manifold $M$ ( $[$ Ha96] $)$. The hermitian elements of the algebra $\mathcal{M}(\mathcal{O})$ are interpreted as observables that can be measured in the "laboratory" $\mathcal{O}$. Accordingly, one requires isotony, i.e., that $\mathcal{O}_{1} \subseteq \mathcal{O}_{2}$ implies $\mathcal{M}\left(\mathcal{O}_{1}\right) \subseteq \mathcal{M}\left(\mathcal{O}_{2}\right)$. Causality enters by the locality assumption that $\mathcal{M}\left(\mathcal{O}_{1}\right)$ and $\mathcal{M}\left(\mathcal{O}_{2}\right)$ commute if $\mathcal{O}_{1}$ and $\mathcal{O}_{2}$ are space-like separated, i.e., cannot correspond with each other. One further assumes a unitary representation $U: G \rightarrow \mathrm{U}(\mathcal{H})$ of a Lie group $G$, acting as a space-time symmetry group on $M$, such that $U(g) \mathcal{M}(\mathcal{O}) U(g)^{*}=\mathcal{M}(g \mathcal{O})$ for $g \in G$. In addition, one assumes a $U(G)$-fixed unit vector $\Omega \in \mathcal{H}$, representing typically a vacuum state of a quantum field. The domains $\mathcal{O} \subseteq M$ for which $\Omega$ is cyclic and separating for $\mathcal{M}(\mathcal{O})$ are of particular relevance. For these domains $\mathcal{O}$, the von Neumann algebra $\mathcal{M}(\mathcal{O})$ specifies a standard subspace $\mathrm{V}(\mathcal{O}) \subseteq \mathcal{H}$ which determines a pair $\left(\Delta_{\mathcal{O}}, J_{\mathcal{O}}\right)$ of modular objects and in particular 
a modular automorphism group $\alpha_{t}(M)=\Delta_{\mathcal{O}}^{-i t / 2 \pi} M \Delta_{\mathcal{O}}^{i t / 2 \pi}$. It is now an interesting question if this modular group is "geometric" in the sense that it is implemented by a one-parameter subgroup of $G$, hence corresponds to a one-parameter group of symmetries of $M$. For the modular conjugation $J_{\mathcal{O}}$, we may likewise ask for the existence of an involutive automorphism $\tau_{G}$ of $G$ and an involution $\tau_{M}$ on $M$ reversing the causal structure, such that

$$
J_{\mathcal{O}} \mathcal{M}(\widetilde{\mathcal{O}}) J_{\mathcal{O}}=\mathcal{M}\left(\tau_{M}(\widetilde{\mathcal{O}})\right), \quad J_{\mathcal{O}} U(g) J_{\mathcal{O}}=U\left(\tau_{G}(g)\right) \quad \text { for } \quad g \in G, \widetilde{\mathcal{O}} \subseteq M,
$$

and that $\tau_{M}$ and $\tau_{G}$ are compatible in the sense that

$$
\tau_{M} \circ g=\tau_{G}(g) \circ \tau_{M} \quad \text { for } \quad g \in G .
$$

We are particularly interested in the geometric realizations of modular groups and involutions in the sense explained above. The present paper contributes to this project by exhibiting large classes of nets of standard subspaces where the action of the modular group is geometric.

To study this question systematically, we first observe that, passing from operator algebras to the corresponding standard subspaces is a tremendous reduction of information, but the net of standard subspaces $\mathrm{V}(\mathcal{O})$ still encodes the geometric features of the original theory and in particular it still reflects the action of the symmetry group $G$ and its extensions by involutions.

Conversely, one can use the functorial process provided by Second Quantization ([Si74] to associate to each standard subspace $\mathrm{V} \subseteq \mathcal{H}$ a pair $\left(\mathcal{R}_{ \pm}(\mathrm{V}), \Omega\right)$, where $\mathcal{R}_{ \pm}(\mathrm{V})$ is a von Neumann algebra on the bosonic/fermionic Fock space $\mathcal{F}_{ \pm}(\mathcal{H})$, for which the vacuum vector is cyclic and separating. This method has been developed by Araki and Woods in the context of free bosonic quantum fields (Ar63, Ar64, AW63, AW68); some of the corresponding fermionic results are more recent (cf. EO73, BJL02]). Other statistics (anyons) are discussed in Schr97 and more recent deformations of this procedure are discussed in Le15, §3]. Throughout this paper we only deal with nets of standard subspaces, but it is important to keep in mind that there are functorial constructions that associate to such nets various types of free quantum fields on homogeneous spaces.

The current interest in standard subspaces arose in the 1990s from the work of Borchers and Wiesbrock ([Bo92, Wi93]). This in turn led to the concept of modular localization in Quantum Field Theory introduced by Brunetti, Guido and Longo in BGL02, BGL93; see also BDFS00] and Le15, LL15] for important applications of this technique.

We start with a unitary representation $(U, \mathcal{H})$ of $G$ which extends to an antiunitary representation of the extended group $G_{\tau}=G \rtimes\left\{\mathbf{1}, \tau_{G}\right\}$, containing $G$ as a subgroup of index 2 . Then $J:=U\left(\tau_{G}\right)$ is a conjugation satisfying $J U(g) J=U\left(\tau_{G}(g)\right)$ for $g \in G$. We then obtain for each pair $\left(h, \tau_{G}\right)$ for which $h$ is fixed by the Lie algebra involution $\tau_{\mathfrak{g}}:=\mathbf{L}\left(\tau_{G}\right)$, a standard subspace $\mathrm{V}:=\mathrm{V}_{\left(h, \tau_{G}, U\right)}$, specified by

$$
J_{\mathrm{V}}=U\left(\tau_{G}\right) \quad \text { and } \quad \Delta_{\mathrm{V}}^{-i t / 2 \pi}=U(\exp t h) \quad \text { for } \quad t \in \mathbb{R} .
$$

This assignment is called the Brunetti-Guido-Longo (BGL) construction (see BGL02). As a consequence, standard subspaces can be associated to antiunitary representations in abundance, but only a few of them carry interesting geometric information. In particular, we would like to understand when a standard subspace of the form $\mathrm{V}_{\left(h, \tau_{G}, U\right)}$ arises from a natural family $\mathrm{V}(\mathcal{O})$ of real subspaces associated to open subsets of a homogeneous space $M=G / P$ and which domains $\mathcal{O} \subseteq M$ (so-called generalized wedge domains) correspond to such standard subspaces. The geometric investigation of such domains in causal symmetric spaces will be pursued further in NÓ20].

In the present paper we develop a method for the construction of nets of standard subspaces for antiunitary representations of Lie groups in spaces of distributions which are boundary values of holomorphic functions. This construction provides for a large class of triples $\left(h, \tau_{G}, U\right)$ a realization of the corresponding standard subspace $\mathrm{V}_{\left(h, \tau_{G}, U\right)}$ as some $\mathrm{V}(\mathcal{W})$, associated to an open subset $\mathcal{W}$ of the group $G$ or of a homogeneous space. We obtain such a realization on three levels: 
(GL) the group level, where $\mathcal{W} \subseteq G$ is an open subsemigroup.

(SL) the level of symmetric spaces, where $\mathcal{W}$ is an open domain in the symmetric space $G / G^{\tau_{G}}$.

(CL) the conformal level, where $\mathcal{W}$ is an open domain in a Jordan space-time, i.e., the universal cover of the conformal completion of a euclidean Jordan algebra $E$. These are the Jordan space-times in the sense of Günaydin Gu93, resp., the simple space-time manifolds in the sense of Mack-de Riese ([MdR07]).

In Physics, these three levels arise for one-dimensional conformal field theory, where $G$ is the 3-dimensional Möbius group Möb $\cong \mathrm{PSL}_{2}(\mathbb{R})$, the corresponding Jordan space-time is the real line, considered as the simply connected covering of the circle, and the corresponding causal symmetric space is the 2-dimensional Anti-de Sitter space $\mathrm{AdS}^{2}$. For the universal cover $G=\widetilde{\mathrm{SO}}_{2, d}(\mathbb{R})_{0}$ of the conformal group of $d$-dimensional Minkowski space, the corresponding Jordan space-time is the simply connected cover of the conformal completion of Minkowski space.

The following table contains some information on the simple hermitian Lie algebras of tube type $\mathfrak{g}$, the corresponding simple euclidean Jordan algebra $E \cong \mathfrak{g}^{1}(h)=\operatorname{ker}(\operatorname{ad} h-\mathbf{1})$, the rank of $E$, which is the real rank of the simple real Lie algebra $\mathfrak{g}$, the subalgebra $\mathfrak{h}=\mathfrak{g}^{0}(h)=$ $\operatorname{ker}(\operatorname{ad} h)$, and the topology of the simple Jordan space-time $M$, considered as a product of $\mathbb{R}$ with a Riemannian symmetric space. Here $E_{6}^{c}$ stands for the simply connected compact Lie group of type $E_{6}$ and $F_{4}^{c} \subseteq E_{6}^{c}$ for a connected subgroup of type $F_{4}$.

\begin{tabular}{||l|l|l|l|l||}
\hline $\mathfrak{g}$ (conf. Lie alg) & $E$ (Jordan alg.) & $\operatorname{rk}(E)$ & $\mathfrak{h}=\mathfrak{g}^{0}(h)$ & $M$ \\
\hline \hline $\mathfrak{s o}_{2, n}(\mathbb{R}), n>2$ & $\mathbb{R}^{1, n-1}$ & 2 & $\mathbb{R} \oplus \mathfrak{s o}_{1, n-1}(\mathbb{R})$ & $\mathbb{R} \times \mathbb{S}^{n-1}$ \\
\hline $\mathfrak{s p}_{2 n}(\mathbb{R})$ & $\operatorname{Sym}_{n}(\mathbb{R})$ & $n$ & $\mathbb{R} \oplus \mathfrak{s l}_{n}(\mathbb{R}) \cong \mathfrak{g l}_{n}(\mathbb{R})$ & $\mathbb{R} \times \mathrm{SU}_{n}(\mathbb{R}) / \mathrm{SO}_{n}(\mathbb{R})$ \\
\hline $\mathfrak{s u}_{n, n}(\mathbb{C})$ & $\operatorname{Herm}_{n}(\mathbb{C})$ & $n$ & $\mathbb{R} \oplus \mathfrak{s l}_{n}(\mathbb{C})$ & $\mathbb{R} \times \mathrm{SU}_{n}(\mathbb{C})$ \\
\hline $\mathfrak{s o}^{*}(4 n)$ & $\operatorname{Herm}_{n}(\mathbb{H})$ & $n$ & $\mathbb{R} \oplus \mathfrak{s l}_{n}(\mathbb{H}) \cong \mathfrak{g l}_{n}(\mathbb{H})$ & $\mathbb{R} \times \mathrm{SU}_{2 n}(\mathbb{C}) / \mathrm{U}_{n}(\mathbb{H})$ \\
\hline $\mathfrak{e}_{7(-25)}$ & $\operatorname{Herm}_{3}(\mathbb{O})$ & 3 & $\mathbb{R} \oplus \mathfrak{e}_{6(-26)}$ & $\mathbb{R} \times E_{6}^{c} / F_{4}^{c}$ \\
\hline
\end{tabular}

Our construction of nets of standard subspaces is based on distribution vectors of unitary representations. To introduce this concept, we first observe that the subspace $\mathcal{H}^{\infty} \subseteq \mathcal{H}$ of vectors $v \in \mathcal{H}$ for which the orbit map $U^{v}: G \rightarrow \mathcal{H}, g \mapsto U(g) v$, is smooth (the smooth vectors) is dense (we assume throughout that $\operatorname{dim} G<\infty$ ). It carries a natural Fréchet topology for which the action of $G$ on this space is smooth ( antilinear functionals $\eta: \mathcal{H}^{\infty} \rightarrow \mathbb{C}$ (the distribution vectors) contains in particular Dirac's kets $\langle\cdot, v\rangle, v \in \mathcal{H}$, so that we obtain a rigged Hilbert space

$$
\mathcal{H}^{\infty} \hookrightarrow \mathcal{H} \hookrightarrow \mathcal{H}^{-\infty},
$$

where $G$ acts on all three spaces by representations denoted $U^{\infty}, U$ and $U^{-\infty}$, respectively, and its Lie algebra $\mathfrak{g}$ acts on $\mathcal{H}^{\infty}$ and $\mathcal{H}^{-\infty}$ by the derived representation (see Appendix $\mathrm{A}$ for details).

To any real subspace $\mathrm{E} \subseteq \mathcal{H}^{-\infty}$ and every open subset $\mathcal{O} \subseteq G$, we associate the real subspace

$$
\mathrm{H}_{\mathrm{E}}(\mathcal{O}):=\overline{\operatorname{span}_{\mathbb{R}}\left\{U^{-\infty}(\varphi) \eta: \varphi \in C_{c}^{\infty}(\mathcal{O}, \mathbb{R}), \eta \in \mathrm{E}\right\}},
$$

where

$$
U^{-\infty}(\varphi)=\int_{G} \varphi(g) U^{-\infty}(g) d g, \quad \varphi \in C_{c}^{\infty}(G)
$$

denotes the integrated representation of the convolution algebra $C_{c}^{\infty}(G)$ of test functions on $G$ on the space $\mathcal{H}^{-\infty}$. This assignment has already two obvious properties:

(Iso) Isotony: $\mathcal{O}_{1} \subseteq \mathcal{O}_{2}$ implies $\mathrm{H}_{\mathrm{E}}\left(\mathcal{O}_{1}\right) \subseteq \mathrm{H}_{\mathrm{E}}\left(\mathcal{O}_{2}\right)$. 
(Cov) Covariance: $U(g) \mathrm{H}_{\mathrm{E}}(\mathcal{O})=\mathrm{H}_{\mathrm{E}}(g \mathcal{O})$.

One of our main results consists in specifying sufficient conditions for (3) to produce standard subspaces including some $\mathrm{V}_{\left(h, \tau_{G}, U\right)}$ obtained from the Brunetti-Guido-Longo construction. Important questions in this context are:

(RS) Does $\mathrm{H}_{\mathrm{E}}(\mathcal{O})$ generate $\mathcal{H}$ for each non-empty open subset $\mathcal{O} \subseteq G$ ? (Reeh-Schlieder property)

(BW) If $\mathrm{H}_{\mathrm{E}}(\mathcal{O})$ is standard, when is its modular group implemented by a one-parameter subgroup of $G$ as in (2)? If this is the case for the one-parameter group generated by $h \in \mathfrak{g}$, we say that $\mathrm{H}_{\mathrm{E}}(\mathcal{O})$ has the Bisognano-Wichmann property with respect to $h$.

A structural property with strong impact in this context is the spectrum condition on the infinitesimal generators $\partial U(x)$ of the one-parameter groups $(U(\exp t x))_{t \in \mathbb{R}}$. This is the requirement that the closed convex cone

$$
C_{U}:=\{x \in \mathfrak{g}:-i \partial U(x) \geq 0\}
$$

(the positive cone of $U$ ) is "large" in the sense that the ideal $\mathfrak{g}_{C_{U}}:=C_{U}-C_{U}$ satisfies $\mathfrak{g}=\mathfrak{g}_{C_{U}}+\mathbb{R} h$. We shall see in Theorem 2.14 that this already implies the Reeh-Schlieder property $(\mathrm{RS})$. Under more specific assumptions on $E$ and $\mathcal{O}$, we show that $\mathrm{H}_{\mathrm{E}}(\mathcal{O})$ is actually standard. We refer to Section 3 for details.

Content of this paper: Section 2 is devoted to properties of smooth and distribution vectors for representations for which $C_{U}$ is pointed and generating, i.e., $\mathfrak{g}_{C_{U}}=\mathfrak{g}$. Then $U$ extends by

$$
U(g \exp (i x))=U(g) e^{i \partial U(x)}
$$

to a representation of the complex Olshanski semigroup $S_{C_{U}}=G \exp \left(i C_{U}\right)$ by contractions. In Subsection 2.1 we study the action of $S_{C_{U}}$ on the space of distribution vectors and establish continuity and holomorphy of orbit maps in the weak-*-topology. This is used in Subsection 2.2 to verify the Reeh-Schlieder property (Theorem 2.14).

In Section 3 we turn to a more specific situation, where $\mathrm{V}=\mathrm{V}_{\left(h, \tau_{G}, U\right)}$ is a standard subspace obtained from the BGL construction, and the semigroup

$$
S_{\mathrm{V}}=\{g \in G: U(g) \mathrm{V} \subseteq \mathrm{V}\}
$$

of endomorphisms of $\mathrm{V}$ is large in the sense that its Lie wedge

$$
\mathbf{L}\left(S_{\mathrm{V}}\right)=\left\{x \in \mathfrak{g}: \exp \left(\mathbb{R}_{+} x\right) \subseteq S_{\mathrm{V}}\right\}
$$

(the set of infinitesimal generators of one-parameter subsemigroups of $S_{\mathrm{V}}$ ) spans the Lie algebra $\mathfrak{g}$. Here we build on the previous work $\mathrm{Ne19}$ Ne19b of the first author on these semigroups. The main results of these two papers are easy to describe. In $N$ Ne19 the Lie wedge $\mathbf{L}\left(S_{\mathrm{V}}\right)$ is calculated. As a consequence of its explicit description, the assumption that it spans $\mathfrak{g}$ implies that ad $h$ defines a 3 -grading in the sense that

$$
\mathfrak{g}=\mathfrak{g}^{1} \oplus \mathfrak{g}^{0} \oplus \mathfrak{g}^{-1} \quad \text { for } \quad \mathfrak{g}^{j}:=\operatorname{ker}\left(\operatorname{ad} h-j \operatorname{id}_{\mathfrak{g}}\right) .
$$

Assuming (5), the semigroup $S_{\mathrm{v}}$ has been completely determined in Ne19b. To describe the structure of $S_{\mathrm{v}}$, let

$$
C_{ \pm}:= \pm C_{U} \cap \mathfrak{g}_{ \pm 1}, \quad \text { and write } \quad G_{\mathrm{V}}=\{g \in G: U(g) \mathrm{V}=\mathrm{V}\}
$$

for the stabilizer group of $\mathrm{v}$ in $G$. Then

$$
S_{\mathrm{V}}=\exp \left(C_{+}\right) G_{\mathrm{V}} \exp \left(C_{-}\right)=G_{\mathrm{V}} \exp \left(C_{+}+C_{-}\right) .
$$


In the setting of (5), we define in Subsection 3.1 the space $\mathrm{V}^{-\infty}$ of distribution vectors associated to the standard subspace $\mathrm{V}$. This requires an extension of the symplectic form on $\mathcal{H}$ to $\mathcal{H}^{\infty} \times \mathcal{H}^{-\infty}$ and the symplectic orthogonal space $\mathrm{V}^{\prime}$ of $\mathrm{V}$, so that we can define $\mathrm{V}^{-\infty}$ as the annihilator of $\mathrm{V}^{\prime} \cap \mathcal{H}^{\infty}$. Here the difficulty is to identify elements of this space. This is achieved in Subsection 3.2, where we extend the following characterization of elements in a standard subspaces from [NÓO20. Prop. 2.1] to distribution vectors: An element $\xi \in \mathcal{H}$ is contained in $\mathrm{V}$ if and only if the orbit map $\alpha^{\xi}: \mathbb{R} \rightarrow \mathcal{H}, \alpha^{\xi}(t):=\Delta_{\mathrm{V}}^{-i t / 2 \pi} \xi$ extends to a continuous map on the closure of the strip

$$
\mathcal{S}_{\pi}=\{z \in \mathbb{C}: 0<\operatorname{Im} z<\pi\},
$$

which is holomorphic on $\mathcal{S}_{\pi}$ and satisfies the boundary relation $\alpha^{\xi}(\pi i)=J_{\mathrm{v}} \xi$. A suitable extension of this requirement to distribution vectors specifies a linear subspace $\mathcal{H}_{\text {ext }, J}^{-\infty} \subseteq \mathcal{H}^{-\infty}$ which is invariant under $U^{-\infty}\left(S_{\mathrm{V}}^{0}\right)$ and $U^{-\infty}\left(C^{\infty}\left(S_{\mathrm{V}}^{0}\right)\right)$. This leads to the inclusion $\mathcal{H}_{\text {ext }, J}^{-\infty} \subseteq \mathrm{V}^{-\infty}$ (Lemma 3.12) which is one of our key tools. We conclude Section 3 with the observation that $\mathcal{H}_{\text {ext }}^{-\infty}$ is invariant under the Lie algebra and discuss the irreducible antiunitary representation of the $a x+b$-group on $L^{2}\left(\mathbb{R}_{+}\right)$in some detail.

The results in Section 3 are valid for general Lie groups whose Lie algebra contains an invariant cone $C$ and an element $h$ defining a 3-grading for which the cones $C_{ \pm}= \pm C \cap \mathfrak{g}^{ \pm 1}$ are generating. To construct representations to which the theory developed in Section 3 applies, we specialize in Section 4 to semisimple Lie groups. Assuming, in addition, that $\mathfrak{g}^{0}$ contains no non-zero ideals, $\mathfrak{g}$ is a direct sum of simple hermitian ideals of tube type and $E:=\mathfrak{g}^{1}$ thus carries the structure of a unital euclidean Jordan algebra such that $C_{+}$is the closure of the open positive cone $E_{+}$of invertible squares in $E$ (see [FK94] and the table above).

This structure is used in Section 4 to show that every irreducible antiunitary representation $(U, \mathcal{H})$ of $G_{\tau}$, which is $C$-positive in the sense that $C \subseteq C_{U}$, can be realized on a Hilbert space $\mathcal{H}_{\rho}$ of holomorphic vector-valued functions on the tube domain $\mathcal{T}:=E+i E_{+}$on which the group $G$ acts as a group of biholomorphic automorphisms, and $\tau_{G}$ corresponds to the antiholomorphic involution $\tau_{E}(z)=-\bar{z}$. The input for this construction is a real irreducible representation $\left(\rho, \mathcal{K}_{\mathbb{R}}\right)$ of the simply connected Lie group $\widetilde{H}$ with Lie algebra $\mathfrak{g}^{0}$ which satisfies a certain positivity condition. Then $\mathcal{H}_{\rho} \subseteq \operatorname{Hol}(\mathcal{T}, \mathcal{K})$, the space of holomorphic functions with values in the complexification $\mathcal{K}$ of $\mathcal{K}_{\mathbb{R}}$. The positivity condition for $\left(\rho, \mathcal{K}_{\mathbb{R}}\right)$ is hard to evaluate explicitly for non-scalar representations, an explicit classification can be derived by combining the classification of unitary highest modules of $\mathfrak{g}$, due to Enright, Howe and Wallach ([EHW83]), with the translation into the present context, carried out in HN01. The main point of this realization is that evaluation in $0 \in \partial \mathcal{T}$ defines a continuous linear map $\mathcal{H}_{\rho}^{\infty} \rightarrow \mathcal{K}$, which embeds $\mathcal{K}$ into $\mathcal{H}^{-\infty}$. In Section 5 we show that this leads to a real subspace $\mathrm{E} \subseteq \mathcal{K}$ contained in $\mathrm{V}^{-\infty}$, so that we can apply Section 3 We then have the identity $\mathrm{V}=\mathrm{H}_{\mathrm{E}}\left(S_{\mathrm{V}}^{0}\right)$ and obtain a net $\mathrm{V}(\mathcal{O}):=\mathrm{H}_{\mathrm{E}}(\mathcal{O})$ of cyclic subspaces associated to non-empty open subsets $\mathcal{O} \subseteq G$. The set of generalized wedge domains in $G$ in the sense of (GL) is the set

$$
\mathcal{W}=\{g S: g \in G\}
$$

of left translates of the connected component $S$ of the open semigroup $S_{\mathrm{V}}^{0}$ containing $e$ in its closure. If $\mathcal{O}$ is contained in a left translate of the semigroup $S_{\mathrm{V}}^{0}$, then the cyclic subspace $\mathrm{V}(\mathcal{O})$ is standard.

As the real subspace $\mathrm{E}$ is invariant under the subgroups $H:=\left\langle\exp _{G} \mathfrak{g}^{0}\right\rangle$ and $P^{-}:=$ $\exp \left(\mathfrak{g}^{-1}\right) H$ of $G$, it easily follows that $\mathrm{V}(\mathcal{O})=\mathrm{V}(\mathcal{O} \cdot H)=\mathrm{V}\left(\mathcal{O} \cdot P^{-}\right)$, so that these nets of cyclic subspaces also define nets on the homogeneous spaces $G / H$ and $G / P^{-}$. Here $G / H$ is a compactly causal symmetric spaces of Cayley type (HÓ97) und $G / P^{-}$is a Jordan space-time in the sense of Günaydin Gu93 and a simple space-time manifold in the sense of Mack-de Riese ([MdR07]).

Perspectives: In the present paper we construct nets of standard subspaces on homogeneous spaces which are associated to unitary representations $(U, \mathcal{H})$ for which the positive cone $C_{U}$ 
is non-trivial. If this cone is trivial, one needs other techniques to specify suitable subspaces $\mathrm{E} \subseteq \mathrm{V}^{-\infty}$. A prototypical example is the action of the Lorentz group $G=\mathrm{SO}_{1, d}(\mathbb{R})_{0}$ on de Sitter space $\mathrm{dS}^{d}$, which is a non-compactly causal symmetric space. In the physics literature one finds several replacements for the spectrum condition that are used to deal with quantum fields on curved spaces such as de Sitter space. Of particular relevance is the "microlocal spectrum condition"; see [TS16] and PEGW19].

We expect that the reflection positivity condition and the KMS condition that characterize the modular objects (see the appendix of [NÓ20 for details) can be used to express a condition on the distribution vectors in $E$ to ensure that the subspace $\mathrm{H}_{\mathrm{E}}(\mathcal{W})$ associated to a "wedge domain" $\mathcal{W} \subseteq M$ is the standard subspace corresponding to the pair $(h, \tau)$.

In the forthcoming paper NÓ20 we develop a theory of generalized wedge domains in causal symmetric spaces that will shed additional light on the class of domains $\mathcal{W} \subseteq M$ for which one may expect a relation like $\mathrm{H}_{\mathrm{E}}(\mathcal{W})=\mathrm{V}$.

In all these constructions, a good understanding of the class of generalized wedge domains is of crucial importance. This motivated the abstract approach to these domains in [MN20, where the set

$$
\mathcal{G}(G):=\left\{(x, \sigma) \in \mathfrak{g} \times\left(G \times\left\{\tau_{G}\right\}\right) \subseteq \mathfrak{g} \times G_{\tau}: \operatorname{Ad}(\sigma) x=x, \sigma^{2}=e\right\}
$$

is studied as a candidate of an index set for nets of standard subspaces. In this context the Euler couples, i.e., those for which ad $x$ is diagonalizable with eigenvalues $\{-1,0,1\}$ and $\operatorname{Ad}(\sigma)=e^{\pi i \text { ad } x}$ are of particular relevance. For instance, the assumptions in Section 3 imply that $(h, \tau)$ is an Euler couple.

Acknowledgment: We thank Roberto Longo for asking us on several occasions to construct quantum field theories on Lie groups. The present paper is a first step in this direction. We are most grateful to Daniel Oeh for several valuable comments on an earlier version of this manuscript and for pointing out an error in Theorem 5.3 .

\section{Notation}

- For a Lie group $G$ with neutral element $e$, the identity component is denoted $G_{0}$. We write $\mathfrak{g}$ for its Lie algebra, Ad: $G \rightarrow \operatorname{Aut}(\mathfrak{g})$ for the adjoint action of $G$ on $\mathfrak{g}$, induced by the conjugation action of $G$ on itself, and $\operatorname{ad} x(y)=[x, y]$ for the adjoint action of $\mathfrak{g}$ on itself.

- For a group action $G \times M \rightarrow M$ and $m \in M$, we write $G^{m}:=\{g \in G: g \cdot m=m\}$ for the stabilizer subgroup of $m$.

- For a connected Lie group $G$, we write $q_{G}: \widetilde{G} \rightarrow G$ for the universal covering morphism and $\eta_{G}: G \rightarrow G_{\mathbb{C}}$ for the universal complexification.

- If $M$ is a smooth manifold, we write $C_{c}^{\infty}(M)$ for the space of complex-valued test functions on $M$, endowed with the natural LF topology, and $C_{c}^{-\infty}(M)$ for the space of antilinear continuous linear functionals on this space, i.e., the space of distributions on $M$.

- We likewise consider tempered distributions $D \in \mathcal{S}^{\prime}(E)$ on a real finite dimensional vector space $E$ as antilinear functionals on the Schwartz space $\mathcal{S}(E)$. The Fourier transform of an $L^{1}$-function $f$ on $E$ is defined by

$$
\widehat{f}(\lambda):=\int_{E} e^{-i \lambda(x)} f(x) d \mu_{E}(x), \quad \lambda \in E^{*},
$$

where $\mu_{E}$ is a Haar measure on $E$. For tempered distributions $D \in \mathcal{S}^{\prime}(E)$, we define the Fourier transform by

$$
\widehat{D}(\varphi):=D(\widetilde{\varphi}), \quad \text { where } \quad \widetilde{\varphi}(\lambda):=\widehat{\varphi}(-\lambda)=\int_{E} e^{i \lambda(x)} \varphi(x) d \mu_{E}(x) .
$$


The distribution $D_{f}(\varphi):=\int_{E} \overline{\varphi(x)} f(x) d \mu_{E}(x)$, defined by an $L^{1}$-function, then satisfies $\widehat{D_{f}}=D_{\widehat{f}}$.

\section{Distribution vectors of $C$-positive representations}

Let $G$ be a Lie group with Lie algebra $\mathfrak{g}$ and $C \subseteq \mathfrak{g}$ a closed convex pointed generating $\operatorname{Ad}(G)$ invariant cone (see Ne99 for a structure theory of these configurations). In this section we study distribution vectors of unitary representations $(U, \mathcal{H})$ of $G$ which are $C$-positive in the sense that $C \subseteq C_{U} \mathbb{1}^{1}$ Then the representation $U: G \rightarrow \mathrm{U}(\mathcal{H})$ extends analytically to a representation of an Olshanski semigroup $S_{C}=G \exp (i C)$.

In Subsection 2.1 we start with some results concerning the action of the semigroup $S_{C}$ on the spaces of smooth and distribution vectors. In particular, we show that the closed semigroup $S_{C}$ acts on the space $\mathcal{H}^{-\infty}$ of distribution vectors with weak-*-continuous orbit maps that are holomorphic on the interior of $S_{C}$. From this we derive the interesting result that every distribution vector $\eta \in \mathcal{H}^{-\infty}$ defines a $G$-right equivariant map $\mathcal{H}^{-\infty} \rightarrow \operatorname{Hol}\left(S_{C}^{0}\right)$ whose range consists of holomorphic functions with distributional boundary values (Theorem 2.7).

In Subsection 2.2 we turn to real subspaces $\mathrm{H}_{\mathrm{E}}(\mathcal{O})$ generated by $U^{-\infty}\left(C_{c}^{\infty}(\mathcal{O}, \mathbb{R})\right) \mathrm{E}$, where $\mathcal{O} \subseteq G$ is open and $\mathrm{E} \subseteq \mathcal{H}^{-\infty}$ is a real subspace. Here the main result is the Reeh-Schlieder property (Theorem 2.14), asserting that $\mathrm{H}_{\mathrm{E}}(\mathcal{O})$ is total in $\mathcal{H}_{\mathrm{E}}(G)$ for every non-empty open subset $\mathcal{O} \subseteq G$ and every real subspace $\mathrm{E} \subseteq \mathcal{H}^{-\infty}$.

\subsection{The semigroup action on distribution vectors}

We start by recalling the construction of the complex Olshanski semigroup $S_{C}$ associated to the invariant cone $C \subseteq \mathfrak{g}$.

Definition 2.1. (Olshanski semigroups) Let $G$ be a connected Lie group with Lie algebra $\mathfrak{g}$ and $C \subseteq \mathfrak{g}$ be a pointed generating $\operatorname{Ad}(G)$-invariant closed convex cone. Then $C$ is elliptic in the sense that, for every element $x \in C^{0}$, the derivation ad $x$ is semisimple with purely imaginary spectrum (Ne99, Prop. VII.3.4(b)]). The corresponding Olshanski semigroup $S_{C}$ is defined as follows.

Let $q_{G}: \widetilde{G} \rightarrow G$ be the universal covering group of $G$ and $\widetilde{G}_{\mathbb{C}}$ be the 1 -connected Lie group with Lie algebra $\mathfrak{g}_{\mathbb{C}}$, so that the universal complexification $\eta_{\widetilde{G}}: \widetilde{G} \rightarrow \widetilde{G}_{\mathbb{C}}$ simply is the canonical morphism of Lie groups for which $\mathbf{L}\left(\eta_{\widetilde{G}}\right): \mathfrak{g} \hookrightarrow \mathfrak{g}_{\mathbb{C}}$ is the inclusion. 2 As $\widetilde{G}_{\mathbb{C}}$ is simply connected, the complex conjugation on $\mathfrak{g}_{\mathbb{C}}$ integrates to an antiholomorphic involution $\sigma: \widetilde{G}_{\mathbb{C}} \rightarrow \widetilde{G}_{\mathbb{C}}$ with $\sigma \circ \eta_{\widetilde{G}}=\eta_{\widetilde{G}}$, and this implies that $\eta_{\widetilde{G}}(\widetilde{G})$ coincides with the subgroup $\left(\widetilde{G}_{\mathbb{C}}\right)^{\sigma}$ of $\sigma$-fixed points in $\widetilde{G}_{\mathbb{C}}$. 3 As $C$ is elliptic, Lawson's Theorem ([Ne99, Thm. XIII.5.6]) asserts that

$$
S_{C}^{\prime}:=\left(\widetilde{G}_{\mathbb{C}}\right)^{\sigma} \exp (i C) \subseteq \widetilde{G}_{\mathbb{C}}
$$

is a closed subsemigroup of $\widetilde{G}_{\mathbb{C}}$ stable under the antiholomorphic involution $s^{*}:=\sigma(s)^{-1}$ and the polar map

$$
\left(\widetilde{G}_{\mathbb{C}}\right)^{\sigma} \times C \rightarrow S_{C}, \quad(g, x) \mapsto g \exp (i x)
$$

is a homeomorphism. Next we observe that ker $\eta_{\widetilde{G}}$ is a discrete subgroup of $\widetilde{G}$ and define $\widetilde{S}_{C}$ as the simply connected covering space of $S_{C}^{\prime}$ and

$$
S_{C}:=\widetilde{S}_{C} / \operatorname{ker}\left(q_{G}\right)
$$

\footnotetext{
${ }^{1}$ In Section 3 this section will be applied to the ideal $\mathfrak{g}_{C}:=C-C$ generated by the invariant cone $C$.

${ }^{2}$ In general the map $\eta_{G}$ is not injective, as the example $G=\widetilde{\mathrm{SL}}_{2}(\mathbb{R})$ with $G_{\mathbb{C}}=\mathrm{SL}_{2}(\mathbb{C})$ shows.

${ }^{3}$ Since $\widetilde{G}_{\mathbb{C}}$ is simply connected, this subgroup is connected by [Lo69, Thm. IV.3.4].
} 
(Ne99, Def. XI.1.11]). The quotient map is denoted $q_{S_{C}}: \widetilde{S}_{C} \rightarrow S_{C}$. It is the universal covering of $S_{C}$. We write

$$
\widetilde{\exp }: \mathfrak{g}+i C \rightarrow \widetilde{S}_{C}
$$

for the continuous lift of the exponential function exp: $\mathfrak{g}+i C=\mathbf{L}\left(S_{C}^{\prime}\right) \rightarrow S_{C}^{\prime} \subseteq \widetilde{G}_{\mathbb{C}}$ and

$$
\exp :=q_{S_{C}} \circ \widetilde{\exp }: \mathfrak{g}+i C \rightarrow S_{C} .
$$

This function extends the exponential function of the group $G$ and, for every $x \in \mathfrak{g}+i C$, the curve $\gamma_{x}(t):=\exp (t x)$ is a continuous one-parameter semigroup of $S_{C}$. Basic covering theory implies that $S_{C}$ inherits an involution given by

$$
(g \exp (i x))^{*}=\exp (i x) g^{-1}=g^{-1} \exp (\operatorname{Ad}(g) i x)
$$

and a homeomorphic polar map $G \times C \rightarrow S_{C},(g, x) \mapsto g \exp (i x)$.

If $C$ has interior points, then the polar map maps $\left(\widetilde{G}_{\mathbb{C}}\right)^{\sigma} \times C^{0}$ diffeomorphically onto the interior $S_{C^{0}}^{\prime}$ of $S_{C}^{\prime}$. Hence $S_{C}^{0}=S_{C^{0}}=G \exp \left(i C^{0}\right)$ is a complex manifold with a holomorphic multiplication, and the exponential function $\mathfrak{g}+i C^{0} \rightarrow S_{C^{0}}$ is holomorphic, whereas the involution $*$ is antiholomorphic ([Ne99, Thm. XI.1.12]).

The following theorem ensures the existence of extensions of a unitary representation $U$ of $G$ to the semigroup $S_{C}$, provided its positive cone $C_{U}$ contains $C$.

Theorem 2.2. (Holomorphic Extension Theorem) If $(U, \mathcal{H})$ is a unitary representation of $G$ satisfying $C \subseteq C_{U}$, then it extends by

$$
U(g \exp (i x))=U(g) e^{i \partial U(x)}, \quad g \in G, x \in C,
$$

to $a$ *-representation of the closed complex Olshanski semigroup $S_{C}=G \exp (i C)$ by contractions on $\mathcal{H}$ which defines a continuous action of $S_{C}$ on $\mathcal{H}$. If $C$ has interior points, then $U$ defines a holomorphic map $S_{C^{0}} \rightarrow B(\mathcal{H})$.

Proof. The existence of the holomorphic extension to $S_{C}$ follows from [Ne99, Thm. XI.2.5] and its weak continuity from [Ne99, Prop. XI.3.7]. As it is a representation by contractions, strong continuity follows from [Ne99, Cor. IV.1.18]. Since the representation of $S_{C}$ on $\mathcal{H}$ is strongly continuous and by contractions, it defines a continuous action of $S_{C}$ on $\mathcal{H}$ because

$$
\left\|U\left(s^{\prime}\right) \xi^{\prime}-U(s) \xi\right\| \leq\left\|U\left(s^{\prime}\right)\left(\xi^{\prime}-\xi\right)\right\|+\left\|\left(U\left(s^{\prime}\right)-U(s)\right) \xi\right\| \leq\left\|\xi^{\prime}-\xi\right\|+\left\|\left(U\left(s^{\prime}\right)-U(s)\right) \xi\right\|
$$

for $\xi, \xi^{\prime} \in \mathcal{H}$ and $s, s^{\prime} \in S_{C}$.

The main purpose of this subsection is to analyze the representation of the semigroup $S_{C}$ on the spaces of smooth and distribution vectors. The first crucial step is to show that $U\left(S_{C}\right)$ leaves $\mathcal{H}^{\infty}$ invariant (Proposition [2.4).

Lemma 2.3. The operators $(U(s))_{s \in S_{C}}$ preserve the dense subspace $\mathcal{H}^{\infty}$. For every smooth vector $\xi \in \mathcal{H}^{\infty}$, the orbit map

$$
U^{\xi}: S_{C} \rightarrow \mathcal{H}^{\infty}, \quad s \mapsto U(s) \xi
$$

is continuous on $S_{C}$ and holomorphic on the interior $S_{C}^{0}$.

Proof. We consider $\mathcal{H}$ as a subspace of $\mathcal{H}^{-\infty}$ as in (85) in Appendix A As the locally convex topology on $\mathcal{H}^{\infty}$ is defined by the topological embedding

$$
\mathcal{H}^{\infty} \hookrightarrow \prod_{D \in \mathcal{U}(\mathfrak{g})} \mathcal{H}, \quad \xi \mapsto(\mathrm{d} U(D) \xi)_{D \in \mathcal{U}(\mathfrak{g})}
$$

(see (83) in Appendix $\mathrm{A}$, we have to show that: 
(a) For every $D \in \mathcal{U}(\mathfrak{g})$ and $\xi \in \mathcal{H}^{\infty}$, we have $f(s):=\mathrm{d} U^{-\infty}(D) U(s) \xi \in \mathcal{H}$ (Lemma A.2).

(b) The function $f: S_{C} \rightarrow \mathcal{H}$ is continuous and holomorphic on $S_{C}^{0}$.

We first observe that the adjoint action of $G$ on $\mathfrak{g}$ extends to a locally finite holomorphic representation of the universal complexification $G_{\mathbb{C}}($ HN12, Thm. 15.1.4]). As the semigroup $S_{C}$ has a natural continuous homomorphism

$$
\eta_{S}: S_{C} \rightarrow G_{\mathbb{C}}, \quad \eta_{S}(g \exp (i x))=\eta_{G}(g) \exp _{G_{\mathbb{C}}}(i x)
$$

which is holomorphic on the interior (Definition 2.1), we thus obtain in particular a continuous representation

$$
\operatorname{Ad}_{\mathbb{C}}: S_{C} \rightarrow \operatorname{Aut}(\mathcal{U}(\mathfrak{g}))
$$

of $S_{C}$ on the complex enveloping algebra $\mathcal{U}(\mathfrak{g})$ which is holomorphic on $S_{C}^{0}$. For $g \in G$ and $D \in \mathcal{U}(\mathfrak{g})$, we have the relation

$$
\mathrm{d} U(D) U(g)=U(g) \mathrm{d} U\left(\operatorname{Ad}\left(g^{-1}\right) D\right): \mathcal{H}^{\infty} \rightarrow \mathcal{H}
$$

We claim that

$$
\mathrm{d} U^{-\infty}(D) U(s)=U(s) \mathrm{d} U\left(\operatorname{Ad}_{\mathbb{C}}(s)^{-1} D\right): \mathcal{H}^{\infty} \rightarrow \mathcal{H}^{-\infty} \quad \text { for } \quad s \in S_{C} .
$$

Note that both sides define linear maps $\mathcal{H}^{\infty} \rightarrow \mathcal{H}^{-\infty}$ and that the right hand side maps into $\mathcal{H}$. To verify (12), we have to show that

$$
\left\langle\xi, \mathrm{d} U^{-\infty}(D) U(s) \eta\right\rangle=\left\langle\xi, U(s) \mathrm{d} U\left(\operatorname{Ad}_{\mathbb{C}}(s)^{-1} D\right) \eta\right\rangle \quad \text { for } \quad \xi, \eta \in \mathcal{H}^{\infty} .
$$

The left hand side equals $\left\langle\mathrm{d} U\left(D^{*}\right) \xi, U(s) \eta\right\rangle$, which is continuous on $S_{C}$ and holomorphic on the interior. For the right hand side we obtain

$$
\left\langle\xi, U(s) \mathrm{d} U\left(\operatorname{Ad}_{\mathbb{C}}(s)^{-1} D\right) \eta\right\rangle=\left\langle U\left(s^{*}\right) \xi, \mathrm{d} U\left(\operatorname{Ad}_{\mathbb{C}}(s)^{-1} D\right) \eta\right\rangle .
$$

Here $U\left(s^{*}\right) \xi$ is continuous on $S_{C}$ and antiholomorphic on $S_{C}^{0}$ and $\operatorname{d} U\left(\operatorname{Ad}_{\mathbb{C}}(s)^{-1} D\right) \eta$ is also continuous on $S_{C}$ and holomorphic on $S_{C}^{0}$. Therefore the right hand side of (13) is continuous on $S_{C}$ and holomorphic on the interior. As both sides of (13) coincide on $G$ by (11), they are equal (티, Lemma A.III.6]). This implies (12), and hence that $\mathrm{d} U^{-\infty}(D) U(s) \mathcal{H}^{\infty} \subseteq \mathcal{H}$. As

$$
\mathcal{H}^{\infty}=\left\{\xi \in \mathcal{H} \subseteq \mathcal{H}^{-\infty}:(\forall D \in \mathcal{U}(\mathfrak{g})) \mathrm{d} U^{-\infty}(D) \xi \in \mathcal{H}\right\}
$$

by Lemma A.2 it follows that $U\left(S_{C}\right)$ preserves $\mathcal{H}^{\infty}$. Since the right hand side of (12) defines a continuous linear map $\mathcal{H}^{\infty} \rightarrow \mathcal{H}$, the definition of the topology on $\mathcal{H}^{\infty}$ by the embedding (10) also shows that the restrictions

$$
U^{\infty}(s):=\left.U(s)\right|_{\mathcal{H}^{\infty}}: \mathcal{H}^{\infty} \rightarrow \mathcal{H}^{\infty}, \quad s \in S_{C},
$$

are continuous linear maps. In particular, we have $f(s) \in \mathcal{H}^{\infty}$ for $s \in S_{C}$. To see that $f$ is continuous, we write it with (12) as

$$
f(s)=U(s) \mathrm{d} U\left(\operatorname{Ad}_{\mathbb{C}}(s)^{-1} D\right) \xi .
$$

As the representation of $S_{C}$ defines a continuous action on $\mathcal{H}$ (Theorem 2.2), the continuity of $f$ follows from the continuity of the map

$$
S_{C} \rightarrow \mathcal{H}, \quad s \mapsto \mathrm{d} U\left(\operatorname{Ad}_{\mathbb{C}}(s)^{-1} D\right) \xi
$$

which actually "extends" to a holomorphic map on all of $G_{\mathbb{C}}$.

That $f$ is holomorphic on $S_{C}^{0}$ follows likewise from the holomorphy of the action of $S_{C}^{0}$ on the complex manifold $\mathcal{H}$ and the holomorphy of (15) on $S_{C}^{0}$. 
With Lemma 2.3 we immediately get:

Proposition 2.4. The prescription

$$
U^{-\infty}(s) \alpha:=\alpha \circ U^{\infty}\left(s^{*}\right) \quad \text { for } \quad \alpha \in \mathcal{H}^{-\infty}, s \in S_{C},
$$

defines a representation of $S_{C}$ on $\mathcal{H}^{-\infty}$ whose orbit maps are weak-*-continuous and weak-*holomorphic on $S_{C}^{0}$.

Although the closed semigroup $S_{C}$ acts on smooth and distribution vectors, its interior $S_{C^{0}}$ has particular regularizing properties which are described in the following two lemmas.

Lemma 2.5. For $s \in S_{C}^{0}$, we have $U(s) \mathcal{H} \subseteq \mathcal{H}^{\infty}$, and the maps

$$
U(s): \mathcal{H} \rightarrow \mathcal{H}^{\infty}, \quad s \in S_{C}^{0},
$$

are continuous.

Proof. Let $\xi \in \mathcal{H}$. Then the orbit map $U^{\xi}: S_{C}^{0} \rightarrow \mathcal{H}, U^{\xi}(s):=U(s) \xi$ is holomorphic, so that its range lies in particular in the space of smooth vectors for $G$. For $D=x_{1} \cdots x_{n} \in \mathcal{U}(\mathfrak{g})$, $x_{j} \in \mathfrak{g}$, we consider the differential operator defined by

$$
D^{R}: C^{\infty}\left(S_{C}^{0}\right) \rightarrow C^{\infty}\left(S_{C}^{0}\right), \quad\left(D^{R} \varphi\right)(s)=\left.\frac{\partial^{n}}{\partial t_{1} \cdots \partial t_{n}}\right|_{t_{j}=0} \varphi\left(\exp \left(t_{n} x_{n}\right) \cdots \exp \left(t_{1} x_{1}\right) s\right) .
$$

That the maps (16) are continuous follows with (83) in Appendix Afrom the fact that, for every $D \in \mathcal{U}(\mathfrak{g})$, the composition $\mathrm{d} U(D) \circ U^{\xi}$ is obtained by applying the right invariant differential operator $D^{R}$ to the smooth functions $g \mapsto U^{\xi}(g s), s \in S_{C}^{0}$.

Lemma 2.6. For $s \in S_{C}^{0}$, we have $U^{-\infty}(s) \mathcal{H}^{-\infty} \subseteq \mathcal{H}^{\infty}$ and the orbit maps

$$
U^{-\infty, \eta}: S_{C}^{0} \rightarrow \mathcal{H}^{\infty}, \quad s \mapsto U^{-\infty}(s) \eta, \quad \eta \in \mathcal{H}^{-\infty},
$$

are holomorphic.

Proof. For $D \in \mathcal{U}(\mathfrak{g})$, let $D^{L}$ denote the corresponding left invariant differential operator. For $x_{1}, \ldots, x_{n} \in \mathfrak{g}$ and $D=x_{1} \cdots x_{n}$, it is defined by

$$
\left(D^{L} \varphi\right)(g)=\left.\frac{\partial^{n}}{\partial t_{1} \cdots \partial t_{n}}\right|_{t_{j}=0} \varphi\left(g \exp \left(t_{1} x_{1}\right) \cdots \exp \left(t_{n} x_{n}\right)\right)
$$

For $\xi \in \mathcal{H}$, the orbit map $U^{\xi}: S_{C}^{0} \rightarrow \mathcal{H}$ is holomorphic and $G$-equivariant with respect to left multiplications in the sense that

$$
U^{\xi} \circ \lambda_{g}=U(g) \circ U^{\xi} \quad \text { for } \quad g \in G, \lambda_{g}(h)=g h,
$$

in particular $U^{\xi}\left(S_{C}^{0}\right) \subseteq \mathcal{H}^{\infty}$. It follows that, for $D \in \mathcal{U}(\mathfrak{g})$, the function

$$
D^{L} U^{\xi}: S_{C}^{0} \rightarrow \mathcal{H}
$$

obtained by applying a left invariant differential operator, is also holomorphic and $G$-equivariant in the sense of (18), hence takes values in $\mathcal{H}^{\infty}$.

Next we observe that, for $\eta \in \mathcal{H}^{\infty}$ and $s \in S_{C}^{0}$, the relation

$$
\left\langle U^{\xi}(s), \eta\right\rangle=\left\langle\xi, U\left(s^{*}\right) \eta\right\rangle
$$


leads for $D:=x_{1} \cdots x_{k}, x_{j} \in \mathfrak{g}$, to

$$
\begin{aligned}
\left\langle\left(D^{L} U^{\xi}\right)(s), \eta\right\rangle & =\left.\frac{\partial^{k}}{\partial t_{1} \cdots \partial t_{k}}\right|_{t_{1}=\cdots=t_{k}=0}\left\langle U^{\xi}\left(s \exp \left(t_{1} x_{1}\right) \cdots \exp \left(t_{k} x_{k}\right)\right), \eta\right\rangle \\
& =\left.\frac{\partial^{k}}{\partial t_{1} \cdots \partial t_{k}}\right|_{t_{1}=\cdots=t_{k}=0}\left\langle U\left(\exp \left(t_{1} x_{1}\right) \cdots \exp \left(t_{k} x_{k}\right)\right) \xi, U\left(s^{*}\right) \eta\right\rangle \\
& =\left\langle\mathrm{d} U^{-\infty}(D) \xi, U\left(s^{*}\right) \eta\right\rangle=\left\langle U^{-\infty}(s) \mathrm{d} U^{-\infty}(D) \xi, \eta\right\rangle .
\end{aligned}
$$

Here we use that $U\left(s^{*}\right) \eta \in \mathcal{H}^{\infty}$ by Lemma 2.3 and that

$$
\left.\frac{\partial^{k}}{\partial t_{1} \cdots \partial t_{k}}\right|_{t_{1}=\cdots=t_{k}=0} U\left(\exp \left(t_{1} x_{1}\right) \cdots \exp \left(t_{k} x_{k}\right)\right) \xi=\mathrm{d} U^{-\infty}(D) \xi \in \mathcal{H}^{-\infty}
$$

holds in the weak-*-topology on $\mathcal{H}^{-\infty}$. For $\xi \in \mathcal{H}$, we thus obtain

$$
\mathcal{H}^{\infty} \ni\left(D^{L} U^{\xi}\right)(s)=U^{-\infty}(s) \mathrm{d} U^{-\infty}(D) \xi .
$$

As $\mathcal{H}^{-\infty}$ is spanned by $\mathrm{d} U^{-\infty}(\mathfrak{g}) \mathcal{H}$ (see Lemma A.2(b)), it follows that $U^{-\infty}(s) \mathcal{H}^{-\infty} \subseteq \mathcal{H}^{\infty}$.

To see that the orbit maps (17) are holomorphic, we have to show that the maps

$$
f: S_{C}^{0} \rightarrow \mathcal{H}, \quad s \mapsto \mathrm{d} U(D) U^{-\infty}(s) \mathrm{d} U^{-\infty}\left(D^{\prime}\right) \xi, \quad \xi \in \mathcal{H}, D, D^{\prime} \in \mathcal{U}(\mathfrak{g}),
$$

are holomorphic because $\mathcal{H}^{-\infty}$ is spanned by $\mathrm{d} U^{-\infty}(\mathcal{U}(\mathfrak{g})) \mathcal{H}($ Lemma $\mathrm{A.2}$ (b)). As

$$
f=D^{R}\left(D^{\prime}\right)^{L} U^{\xi}: S_{C}^{0} \rightarrow \mathcal{H}
$$

and $U^{\xi}$ is holomorphic, $f$ is holomorphic as well.

The following theorem shows that any distribution vector generates a subrepresentation that can be realized in holomorphic functions on $S_{C}^{0}$ with distributional boundary values.

Theorem 2.7. (Realization in holomorphic functions) Let $\eta \in \mathcal{H}^{-\infty}$. By

$$
j_{\eta}^{S}: \mathcal{H}^{-\infty} \rightarrow \operatorname{Hol}\left(S_{C}^{0}\right), \quad j_{\eta}^{S}(\alpha)(s):=\alpha\left(U^{-\infty}\left(s^{*}\right) \eta\right),
$$

we obtain a map which intertwines $U^{-\infty}$ with the action of $S_{C}$ on $\operatorname{Hol}\left(S_{C}^{0}\right)$ by right translations. Every function $j_{\eta}^{S}(\alpha) \in \operatorname{Hol}\left(S_{C}^{0}\right)$ has the distributional boundary value $j_{\eta}^{\vee}(\alpha):=j_{\eta}(\alpha)^{\vee} \in$ $C^{-\infty}(G)$ in the sense that

$$
j_{\eta}^{\vee}(\alpha)(\varphi)=\lim _{s \rightarrow e} \int_{G} \overline{\varphi(g)} j_{\eta}^{S}(\alpha)(g s) d g \quad \text { for } \quad \varphi \in C_{c}^{\infty}(G) .
$$

Proof. (a) As $U^{-\infty}(s) \eta \in \mathcal{H}^{\infty}$ for $s \in S_{C}^{0}$ by Lemma 2.6, the function $j_{\eta}^{S}(\alpha): S_{C}^{0} \rightarrow \mathbb{C}$ is defined for each distribution vector $\alpha$. That it is holomorphic follows from the holomorphy of the orbit maps (17) in Lemma 2.6 and the antilinearity of $\alpha$ on $\mathcal{H}^{\infty}$.

(b) By definition (see (93)), the left hand side of (19) equals

$$
j_{\eta}^{\vee}(\alpha)(\varphi)=\alpha\left(U^{-\infty}\left(\varphi^{\vee}\right) \eta\right) \quad \text { for } \quad \varphi^{\vee}(g):=\Delta_{G}(g)^{-1} \varphi\left(g^{-1}\right) .
$$

Using that

$$
\int_{G} \varphi(g) U\left(g^{-1}\right) d g=\int_{G} \varphi^{\vee}(g) U(g) d g=U\left(\varphi^{\vee}\right),
$$

we obtain for the integral on the right hand side of (19)

$$
\int_{G} \overline{\varphi(g)} \alpha\left(U^{-\infty}\left(s^{*} g^{-1}\right) \eta\right) d g=\alpha\left(U^{-\infty}\left(s^{*}\right) U^{-\infty}\left(\varphi^{\vee}\right) \eta\right)=\left(U^{-\infty}(s) \alpha\right)\left(U^{-\infty}\left(\varphi^{\vee}\right) \eta\right) .
$$

Evaluating on the smooth vector $U^{-\infty}\left(\varphi^{\vee}\right) \eta$, the assertion now follows from the weak-*continuity of the map $S_{C} \rightarrow \mathcal{H}^{-\infty}, s \mapsto U^{-\infty}(s) \alpha$ (Proposition 2.4). 


\subsection{Real subspaces generated by distribution vectors}

In this subsection we turn to real subspaces of $C$-positive unitary representations $(U, \mathcal{H})$ of $G$. Our main result is the Reeh-Schlieder property (Theorem 2.14). We shall use the following terminology concerning real subspaces of complex Hilbert spaces, which is inspired by cyclic and separating vectors for Neumann algebras, as defined in the introduction.

Definition 2.8. Let $\mathrm{H}$ be a closed real subspace of the complex Hilbert space $\mathcal{H}$.

(a) We write

$$
\mathrm{H}^{\prime}:=\{\xi \in \mathcal{H}:(\forall \eta \in \mathrm{H}) \operatorname{Im}\langle\xi, \eta\rangle=0\}
$$

for its symplectic orthogonal space and note that $\mathrm{H}=\mathrm{H}^{\prime \prime}$ follows from the closedness of $\mathrm{H}$.

(b) $\mathcal{H}$ is said to be

- cyclic if $\mathrm{H}+i \mathrm{H}$ is dense in $\mathcal{H}$.

- separating if $\mathrm{H} \cap i \mathrm{H}=\{0\}$. As $\mathrm{H} \cap i \mathrm{H}=\left(\mathrm{H}^{\prime}+i \mathrm{H}^{\prime}\right)^{\prime}$, this is equivalent to is $\mathrm{H}^{\prime}$ being cyclic.

- standard if it is cyclic and separating.

Definition 2.9. Let $(U, \mathcal{H})$ be a unitary representation of $G$. If $\mathrm{E} \subseteq \mathcal{H}^{-\infty}$ is a real linear subspace, then we consider for an open subset $\mathcal{O} \subseteq G$ the subspaces

$$
\mathrm{H}_{\mathrm{E}}(\mathcal{O}):=\overline{\operatorname{span}_{\mathbb{R}}\left(U^{-\infty}\left(C_{c}^{\infty}(\mathcal{O}, \mathbb{R})\right) \mathrm{E}\right)} \subseteq \mathcal{H}_{\mathrm{E}}(\mathcal{O}):=\overline{\operatorname{span}_{\mathbb{C}}\left(U^{-\infty}\left(C_{c}^{\infty}(\mathcal{O})\right) \mathrm{E}\right)} \subseteq \mathcal{H}
$$

For $\eta \in \mathcal{H}^{-\infty}$, we also put

$$
\mathrm{H}_{\eta}(\mathcal{O}):=\mathrm{H}_{\mathbb{R} \eta}(\mathcal{O}) \quad \text { and } \quad \mathcal{H}_{\eta}(\mathcal{O}):=\mathcal{H}_{\mathbb{R} \eta}(\mathcal{O}) .
$$

By construction and (80) in Appendix $\mathrm{A}$ we have

$$
U(g) \mathrm{H}_{\mathrm{E}}(\mathcal{O})=\mathrm{H}_{\mathrm{E}}(g \mathcal{O}) \quad \text { for } \quad g \in G, \mathcal{O} \subseteq G .
$$

In this subsection we show that, if the cone $C_{U}$ has interior points, then $\mathcal{H}_{\eta}(\mathcal{O})=\mathcal{H}_{\eta}(G)$ for every non-empty open subset $\mathcal{O} \subseteq G$ (Reeh-Schlieder property). If $\eta$ is cyclic, i.e., $\mathcal{H}_{\eta}(G)=\mathcal{H}$, this means that $\mathrm{H}_{\eta}(\mathcal{O})$ is a cyclic real subspace.

Lemma 2.10. (Fragmentation Lemma) Let $\emptyset \neq \mathcal{O} \subseteq G$ be open. Then the following assertions hold:

(a) If $P \subseteq G$ is a closed subgroup, then

(i) every test function $\varphi \in C_{c}^{\infty}(\mathcal{O} P, \mathbb{R})$ is a finite sum of test functions of the form

$$
\psi \circ \rho_{p}: G \rightarrow \mathbb{C}, \quad g \mapsto \psi(g p), \quad \psi \in C_{c}^{\infty}(\mathcal{O}, \mathbb{R}), p \in P .
$$

(ii) every test function $\varphi \in C_{c}^{\infty}(P \mathcal{O}, \mathbb{R})$ is a finite sum of test functions of the form

$$
\psi \circ \lambda_{p}: G \rightarrow \mathbb{C}, \quad g \mapsto \psi(p g), \quad \psi \in C_{c}^{\infty}(\mathcal{O}, \mathbb{R}), p \in P .
$$

(b) Every $\varphi \in C_{c}^{\infty}(G, \mathbb{R})$ is a finite sum $\sum_{j=1}^{n} \varphi_{j} \circ \lambda_{g_{j}}$ with $\varphi_{j} \in C_{c}^{\infty}(\mathcal{O}, \mathbb{R})$ and $g_{j} \in G$.

Proof. (a)(i) The family $(\mathcal{O} p)_{p \in P}$ is an open cover of the compact $\operatorname{subset} \operatorname{supp}(\varphi)$, so that there exist $p_{1}, \ldots, p_{n} \in P$ with

$$
\operatorname{supp}(\varphi) \subseteq \mathcal{O} p_{1} \cup \cdots \cup \mathcal{O} p_{n}
$$

Let $\chi_{0}, \ldots, \chi_{n}$ be a smooth partition of unity subordinated to the open cover

$$
G \backslash \operatorname{supp}(\varphi), \quad \mathcal{O} p_{1}, \ldots, \mathcal{O} p_{n}
$$

of $G$. Then $\varphi=\sum_{j=1}^{n} \varphi_{j}$, where $\varphi_{j}:=\chi_{j} \varphi$ satisfies $\operatorname{supp}\left(\varphi_{j}\right) \subseteq \mathcal{O} p_{j}$. Then $\psi_{j}:=\varphi_{j} \circ \rho_{p_{j}} \in$ $C_{c}^{\infty}(\mathcal{O}, \mathbb{R})$ and $\varphi=\sum_{j=1}^{n} \psi_{j} \circ \rho_{p_{j}^{-1}}$

(a)(ii) and (b) are proved along the same lines. For (b), we use the open cover $(g \mathcal{O})_{g \in G}$ of the group $G$. 
Lemma 2.11. Let $(U, \mathcal{H})$ be a unitary representation of $G$, let $\mathrm{E} \subseteq \mathcal{H}^{-\infty}$ be a real linear subspace, $P \subseteq G$ a closed subgroup and $\emptyset \neq \mathcal{O} \subseteq G$. Then the following assertions hold:

(a) $\mathrm{H}_{\mathrm{E}}(\mathcal{O} P)=\mathrm{H}_{\mathrm{E}}(\mathcal{O})$ if $\mathrm{E}$ is P-invariant.

(b) $\mathrm{H}_{\mathrm{E}}(P \mathcal{O})$ is the closed real span of $U(P) \mathrm{H}_{\mathrm{E}}(\mathcal{O})$.

(c) The real subspace spanned by $U(G) \mathrm{H}_{\mathrm{E}}(\mathcal{O})$ is dense in $\mathrm{H}_{\mathrm{E}}(G)$.

Proof. (a) The inclusion $\mathrm{H}_{\mathrm{E}}(\mathcal{O}) \subseteq \mathrm{H}_{\mathrm{E}}(\mathcal{O} P)$ is trivial. Conversely, for $\varphi=\psi \circ \rho_{p}, \psi \in C_{c}^{\infty}(\mathcal{O})$ and $p \in P$, we obtain with (81) in Appendix

$$
U^{-\infty}(\varphi) \mathrm{E}=U^{-\infty}\left(\psi \circ \rho_{p}\right) \mathrm{E}=\Delta_{G}(p)^{-1} U^{-\infty}(\psi) U^{-\infty}\left(p^{-1}\right) \mathrm{E}=U^{-\infty}(\psi) \mathrm{E} \subseteq \mathrm{E} .
$$

Hence the assertion follows from Lemma 2.10(a).

(b) From (23) we know that $U(p) \mathrm{H}_{\mathrm{E}}(\mathcal{O})=\mathrm{H}_{\mathrm{E}}(p \mathcal{O}) \subseteq \mathrm{H}_{\mathrm{E}}(P \mathcal{O})$ for $p \in P$. Now the assertion follows from Lemma 2.10(b).

(c) is an immediate consequence of (b), applied with $P=G$.

The following lemma is of particular interest for semidirect products such as the Poincaré group.

Lemma 2.12. Let $G=L \ltimes_{\sigma} N$ be a semidirect product group and $(U, \mathcal{H})$ be a unitary representation of $G$. We write $U_{N}:=\left.U\right|_{N}$ for the restriction to $N$. Suppose that $\mathrm{E} \subseteq \mathcal{H}^{-\infty}\left(U_{N}\right) \subseteq$ $\mathcal{H}^{-\infty}(U)$ is an L-invariant real subspace. Then we have for every non-empty open $\sigma(L)$ invariant subset $\mathcal{O} \subseteq N$ the relation

$$
\mathrm{H}_{\mathrm{E}}^{N}(\mathcal{O}):=\overline{\operatorname{span}_{\mathbb{R}} U^{-\infty}\left(C_{c}^{\infty}(\mathcal{O}, \mathbb{R})\right) \mathrm{E}}=\mathrm{H}_{\mathrm{E}}(\mathcal{O} L)
$$

Proof. First we show that $\mathrm{H}_{\mathrm{E}}^{N}(\mathcal{O}) \subseteq \mathrm{H}_{\mathrm{E}}(\mathcal{O} L)$. As subsets of $G$, we have $\mathcal{O} L=L \mathcal{O}$, and the multiplication map $L \times \mathcal{O} \rightarrow L \mathcal{O}$ is a diffeomorphism onto an open subset of $G$. On $L \mathcal{O}$ we thus have test functions $\varphi \in C_{c}^{\infty}(L \mathcal{O})$ of the form

$$
\varphi_{n}(l, x)=\delta_{n}(l) \psi(x), \quad \text { where } \quad \psi \in C_{c}^{\infty}(\mathcal{O}, \mathbb{R}),
$$

and $\left(\delta_{n}\right)_{n \in \mathbb{N}}$ is a $\delta$-sequence in $C_{c}^{\infty}(L, \mathbb{R})$ (See Appendix A.1). A left invariant Haar measure of $G$ is given by

$$
d \mu_{G}(l, n)=d \mu_{L}(l) d \mu_{N}(n),
$$

where $\mu_{L}$ and $\mu_{N}$ are left invariant Haar measures on $L$ and $N$, respectively. Then

$$
U\left(\varphi_{n}\right)=\int_{\mathcal{O}} \int_{L} \delta_{n}(l) U(l) \psi(x) U(x) d \mu_{L}(l) d \mu_{N}(x)=U\left(\delta_{n}\right) U(\psi)
$$

leads for $\eta \in \mathrm{E} \subseteq \mathcal{H}^{-\infty}\left(U_{N}\right)$ to

$$
U^{-\infty}\left(\varphi_{n}\right) \eta=U\left(\delta_{n}\right) U^{-\infty}(\psi) \eta \rightarrow U^{-\infty}(\psi) \eta
$$

for $n \rightarrow \infty$. We conclude that $\mathrm{H}_{\mathrm{E}}^{N}(\mathcal{O}) \subseteq \mathrm{H}_{\mathrm{E}}(\mathcal{O} L)$.

To verify the converse inclusion, we recall that the functions

$$
\varphi(l, x)=\varphi_{L}(l) \varphi_{N}(x) \quad \text { where } \quad \varphi_{L} \in C_{c}^{\infty}(L, \mathbb{R}), \varphi_{N} \in C_{c}^{\infty}(\mathcal{O}, \mathbb{R})
$$

span a dense subspace of $C_{c}^{\infty}(L \mathcal{O})$ (Tr67, Thm. 5.16]). In view of (24), it thus suffices to show that,

$$
U\left(\varphi_{L}\right) U^{-\infty}\left(\varphi_{N}\right) \mathrm{E} \subseteq U\left(\varphi_{L}\right) \mathrm{H}_{\mathrm{E}}^{N}(\mathcal{O}) \subseteq \mathrm{H}_{\mathrm{E}}^{N}(\mathcal{O}) .
$$

The first inclusion is trivial, and the second one follows from the $L$-invariance and the closedness of $\mathrm{H}_{\mathrm{E}}^{N}(\mathcal{O})$, which in turn follows from the $\sigma(L)$-invariance of $\mathcal{O}$. 


\subsection{The Reeh-Schlieder property}

The following lemma is our key tool in the derivation of the Reeh-Schlieder property.

Lemma 2.13. Let $(U, \mathcal{H})$ be a unitary representation of the connected Lie group $G$ for which the positive cone $C_{U}$ has interior points. If $\xi, \eta \in \mathcal{H}$ are such that the matrix coefficient

$$
U^{\xi, \eta}: G \rightarrow \mathbb{C}, \quad g \mapsto\langle\xi, U(g) \eta\rangle
$$

vanishes on an open subset of $G$, then $U^{\xi, \eta}=0$ on $G$.

Proof. Passing to the quotient Lie group $G / \operatorname{ker}(U)$, we may w.l.o.g. assume that $U$ is injective. Then the closed convex cone $C_{U} \subseteq \mathfrak{g}$ is pointed, and by assumption it is also generating, so that it defines a closed complex Olshanski semigroup $S_{C_{U}}=G \exp \left(i C_{U}\right)$ (Definition 2.1), and the matrix coefficient $U^{\xi, \eta}$ extends to a continuous function on $S_{C_{U}}$ which is holomorphic on the interior $S_{C_{U}}^{0}=G \exp \left(i C_{U}^{0}\right)$, which is a complex manifold (Theorem 2.2).

Suppose that $U^{\xi, \eta}$ vanishes on the non-empty open subset $\mathcal{O}$. Replacing $\eta$ by $U\left(g_{0}\right) \eta$ for some $g_{0} \in \mathcal{O}$, we may assume that $e \in \mathcal{O}$. The exponential function exp: $\mathfrak{g}+i C_{U} \rightarrow S_{C_{U}}$ is continuous, and holomorphic on $\mathfrak{g}+i C_{U}^{0}$. Therefore the function $U^{\xi, \eta} \circ \exp : \mathfrak{g} \rightarrow \mathbb{C}$ extends to a continuous function on the closed wedge $\mathfrak{g}+i C_{U}$, holomorphic on the interior and vanishing on $\exp ^{-1}(\mathcal{O}) \neq \emptyset$. Now Ne99, Lemma A.III.6] implies that $U^{\xi, \eta} \circ \exp =0$. Therefore the regularity of the exponential function $\mathfrak{g}+i C_{U}^{0} \rightarrow S_{C_{U}}^{0}$ near 0 implies that $U^{\xi, \eta}=0$ on $S_{C_{U}}$, and hence its restriction to $G$ vanishes.

The following theorem is the main result of this section. It is a key tool for geometric constructions of standard subspaces for unitary representations satisfying a spectrum condition, such as $C \subseteq C_{U}$.

Theorem 2.14. (Reeh-Schlieder property) Let $\emptyset \neq \mathcal{O} \subseteq G$ be an open subset and $(U, \mathcal{H})$ be a unitary representation of the connected Lie group $G$ for which the positive cone $C_{U}$ has interior points. Then $\mathrm{H}_{\mathrm{E}}(\mathcal{O})$ is total in $\mathcal{H}_{\mathrm{E}}(G)$ for every real subspace $\mathrm{E} \subseteq \mathcal{H}^{-\infty}$.

Proof. Suppose that $\xi \in \mathcal{H}_{\mathrm{E}}(G)$ is orthogonal to $\mathrm{H}_{\mathrm{E}}(\mathcal{O})$ and let $\varphi \in C_{c}^{\infty}(\mathcal{O}, \mathbb{R})$. Then $\operatorname{supp}(\varphi)$ is a compact subset of $\mathcal{O}$, so that there exists an open $e$-neighborhood $V \subseteq G$ with $V \operatorname{supp}(\varphi) \subseteq \mathcal{O}$. For $g \in V$ and $\eta \in \mathrm{E}$, we then have by (80) in Appendix $\mathrm{A}$

$$
\xi \perp U(g) U^{-\infty}(\varphi) \eta=U^{-\infty}\left(\lambda_{g} \varphi\right) \eta \quad \text { because } \quad \operatorname{supp}\left(\lambda_{g} \varphi\right)=g \operatorname{supp}(\varphi) \subseteq \mathcal{O} .
$$

If follows that, for $\eta^{\prime}:=U^{-\infty}(\varphi) \eta \in \mathcal{H}$, the continuous function

$$
U^{\xi, \eta^{\prime}}: G \rightarrow \mathbb{C}, \quad g \mapsto\left\langle\xi, U(g) \eta^{\prime}\right\rangle
$$

vanishes on the identity neighborhood $V$, and by Lemma 2.13 it vanishes on $G$. We conclude that $\xi \perp U(G) \mathrm{H}_{\mathrm{E}}(\mathcal{O})$, so that Lemma 2.11 shows that $\xi=0$.

\subsection{Support properties of corresponding distributions}

Although we will not use them below, we record some immediate consequences of our discussion for supports of certain distributions. We expect these results to become relevant in subsequent work. For the abelian case, we refer to [NÓO20] for more details.

From Theorem 2.14 we immediately obtain the following criterion for $\mathrm{H}_{E}(\mathcal{O})$ to be standard in terms of support properties of distributions.

Corollary 2.15. Let $\mathcal{O}, \mathcal{O}^{\prime} \subseteq G$ be two non-empty open subsets, let $(U, \mathcal{H})$ be a unitary representation with $C_{U}^{0} \neq \emptyset$, let $\eta \in \mathcal{H}^{-\infty}$, and consider the distribution $D=j_{\eta}(\eta) \in C^{-\infty}(G)$ (see (86) in Appendix A]. Then the following assertions hold: 
(a) $\mathrm{H}_{\eta}\left(\mathcal{O}^{\prime}\right) \subseteq \mathrm{H}_{\eta}(\mathcal{O})^{\prime}$ is equivalent to

$$
\operatorname{Im} D\left(\psi^{*} * \varphi\right)=0 \quad \text { for } \quad \varphi \in C_{c}^{\infty}(\mathcal{O}, \mathbb{R}), \psi \in C_{c}^{\infty}\left(\mathcal{O}^{\prime}, \mathbb{R}\right) .
$$

If this is the case, then $\mathrm{H}_{\eta}(\mathcal{O})$ is a standard subspace of $\mathcal{H}_{\eta}(G)$.

(b) Suppose that $\mathcal{O} \subseteq G$ is an open subsemigroup with $e \in \overline{\mathcal{O}}$. Then

$$
\mathrm{H}_{\eta}\left(\mathcal{O}^{-1}\right) \subseteq \mathrm{H}_{\eta}(\mathcal{O})^{\prime} \quad \Leftrightarrow \quad \operatorname{supp}(\operatorname{Im} D) \subseteq G \backslash \mathcal{O} .
$$

Proof. (a) From Theorem 2.14 we infer that $\mathrm{H}_{\eta}(\mathcal{O})$ is total in $\mathcal{H}_{\eta}(G)$. With the notation from (87) and (90) in Appendix $\$$ w we see that

$$
\operatorname{Im} D\left(\psi^{*} * \varphi\right)=\operatorname{Im}\langle\varphi * D, \psi * D\rangle_{\mathcal{H}_{D}}=\operatorname{Im}\left\langle U^{-\infty}(\varphi) \eta, U^{-\infty}(\psi) \eta\right\rangle
$$

vanishes for all $\varphi \in C_{c}^{\infty}(\mathcal{O}, \mathbb{R})$ and $\psi \in C_{c}^{\infty}\left(\mathcal{O}^{\prime}, \mathbb{R}\right)$ if and only if $\mathrm{H}_{\eta}\left(\mathcal{O}^{\prime}\right) \subseteq \mathbf{H}_{\eta}(\mathcal{O})^{\prime}$. As the real subspace $\mathrm{H}_{\eta}\left(\mathcal{O}^{\prime}\right)$ is cyclic in $\mathcal{H}_{\eta}(G)$ (Theorem 2.14), the above condition implies that the subspace $\mathrm{H}_{\eta}(\mathcal{O})$ is also separating, hence standard.

(b) For $\varphi \in C_{c}^{\infty}(\mathcal{O}, \mathbb{R})$ and $\psi \in C_{c}^{\infty}\left(\mathcal{O}^{\prime}, \mathbb{R}\right)$, the convolution product $\psi^{*} * \varphi$ is a test function on $\mathcal{O}$. Using a $\delta$-sequence in $C_{c}^{\infty}(\mathcal{O}, \mathbb{R})$, it follows that (25) is equivalent to $\operatorname{Im}(D)$ vanishing on $C_{c}^{\infty}(\mathcal{O}, \mathbb{R})$, which means that $\operatorname{supp}(\operatorname{Im} D) \subseteq G \backslash \mathcal{O}$.

Corollary 2.16. Let $G$ be a connected Lie group, $D \in C^{-\infty}(G)$ be a positive definite distribution, and $\left(U_{D}, \mathcal{H}_{D}\right)$ be the corresponding unitary representation of $G$ on the Hilbert subspace $\mathcal{H}_{D} \subseteq C^{-\infty}(G)$. If $C_{U}^{0} \neq \emptyset$ then, for any non-empty open subset $\mathcal{O} \subseteq G$, we have:

(1) The real subspace $\mathrm{H}_{D}(\mathcal{O}):=\overline{C_{c}^{\infty}(\mathcal{O}, \mathbb{R}) * D}$ is cyclic in $\mathcal{H}_{D}$.

(2) If $\mathcal{O}$ is an open subsemigroup with $e \in \overline{\mathcal{O}}$ and $\operatorname{Im} D$ vanishes on $G \backslash \mathcal{O}$, then $\mathrm{H}_{D}(\mathcal{O})$ is a standard subspace of $\mathcal{H}_{D}$ with $\mathrm{H}_{D}\left(\mathcal{O}^{-1}\right) \subseteq \mathrm{H}_{D}(\mathcal{O})^{\prime}$.

Lemma 2.17. Let $\eta \in \mathcal{H}^{-\infty}$ and $\mathcal{O} \subseteq G$ be an open subset for which $\mathrm{H}_{\eta}(\mathcal{O})$ is total in $\mathcal{H}$. If $\alpha \in \mathcal{H}^{-\infty}$ is such that the distribution $j_{\eta}(\alpha)$ vanishes on the subset $V \cdot \mathcal{O}$, for an e-neighborhood $V$ in $G$, then $\alpha=0$.

Proof. Let $\left(\delta_{n}\right)_{n \in \mathbb{N}}$ be a $\delta$-sequence in $C_{c}^{\infty}(G, \mathbb{R})$ and $\varphi \in C_{c}^{\infty}(\mathcal{O}, \mathbb{R})$. Then $\delta_{n} * \varphi \in C_{c}^{\infty}(V \mathcal{O}, \mathbb{R})$ if $n$ is sufficiently large. We therefore have

$$
0=j_{\eta}(\alpha)\left(\delta_{n} * \varphi\right)=\alpha\left(U^{-\infty}\left(\delta_{n} * \varphi\right) \eta\right)=\alpha\left(U^{-\infty}\left(\delta_{n}\right) U^{-\infty}(\varphi) \eta\right)=\left\langle U^{-\infty}(\varphi) \eta, U^{-\infty}\left(\delta_{n}^{*}\right) \alpha\right\rangle .
$$

As $\mathrm{H}_{\eta}(\mathcal{O})$ is total, $U^{-\infty}\left(\delta_{n}\right) \alpha=0$ if $n$ is sufficiently large, and since this sequence converges to $\alpha$ in $\mathcal{H}^{-\infty}$, we obtain $\alpha=0$.

Proposition 2.18. (Full support property) Suppose that $\eta \in \mathcal{H}^{-\infty}$ is such that $\mathrm{H}_{\eta}(\mathcal{O})$ is total in $\mathcal{H}_{\eta}(G)$ for every non-empty open subset $\mathcal{O} \subseteq G$. If $\alpha \in \mathcal{H}^{-\infty}$ is such that $\operatorname{supp}\left(j_{\eta}(\alpha)\right) \neq G$, then $\alpha=0$.

Proof. If $\operatorname{supp}\left(j_{\eta}(\alpha)\right) \neq G$, then there exists a non-empty open subset $\mathcal{O}_{0} \subseteq G$ on which $j_{\eta}(\alpha)$ vanishes. Let $\mathcal{O} \subseteq \mathcal{O}_{0}$ be a non-empty open relatively compact subset. Then there exists an identity neighborhood $V \subseteq G$ with $V \mathcal{O} \subseteq \mathcal{O}_{0}$. As $\mathrm{H}_{\eta}(\mathcal{O})$ is total in $\mathcal{H}$, Lemma 2.17 implies that $\alpha=0$.

The following corollary generalizes Lemma 2.11 to distributional matrix coefficients.

Corollary 2.19. For any unitary representation $\left(U, \mathcal{H}_{D}\right)$ on a subspace $\mathcal{H}_{D} \subseteq C^{-\infty}(G)$ for which $C_{U}^{0} \neq \emptyset$, any non-zero distribution $D^{\prime} \in \mathcal{H}_{D}$ satisfies $\operatorname{supp}\left(D^{\prime}\right)=G$.

Proof. This follows by combining Theorem 2.14 with Proposition 2.18 


\section{$3 \quad C$-positive representation of 3 -graded Lie groups}

After the preparations in Section 2, we now turn to standard subspaces $\mathrm{V}=\mathrm{V}_{\left(h, \tau_{G}, U\right)}$ specified by the BGL construction for an antiunitary representation $(U, \mathcal{H})$ of $G_{\tau}=G \rtimes\left\{\mathbf{1}, \tau_{G}\right\}$ as in (2) by

$$
J_{\mathrm{V}}=U\left(\tau_{G}\right) \quad \text { and } \quad \Delta_{\mathrm{V}}^{-i t / 2 \pi}=U(\exp t h) \quad \text { for } \quad t \in \mathbb{R} .
$$

In this section we assume that $\mathrm{V}$ is regular in the sense that the Lie wedge $\mathbf{L}\left(S_{\mathrm{V}}\right)$ has interior points. This leads us to assumption (B4) below ([Ne19, Thm. 4.4]).

We now consider the following setting which is a non-abelian generalization of [NÓO20]:

(B1) $G$ is a connected Lie group with Lie algebra $\mathfrak{g}$.

(B2) $\tau_{G} \in \operatorname{Aut}(G)$ is an involution and $h \in \mathfrak{g}$ is fixed by $\tau=\mathbf{L}\left(\tau_{G}\right) \in \operatorname{Aut}(\mathfrak{g})$.

(B3) $C \subseteq \mathfrak{g}$ is a pointed closed convex cone invariant under $\operatorname{Ad}(G)$ and $-\tau$, and $\mathfrak{g}=\mathfrak{g}_{C}+\mathbb{R} h$, where $\mathfrak{g}_{C}=C-C$ is the ideal generated by $C$. We write $G_{C} \unlhd G$ for the connected normal subgroup corresponding to $\mathfrak{g}_{C}$ and $A:=\exp (\mathbb{R} h)$. If $h \in \mathfrak{g}$, then $G=G_{C}$, and if $h \notin \mathfrak{g}$, then $G=G_{C} A$.

(B4) $h$ is an Euler element, i.e., ad $h$ is diagonalizable with eigenvalues $\{-1,0,1\}$, and $\tau=$ $\left.e^{\pi i \text { ad } h}\right|_{\mathfrak{g}}$.

Then $\mathfrak{g}=\mathfrak{g}^{-1} \oplus \mathfrak{g}^{0} \oplus \mathfrak{g}^{1}$, where $\left.\tau\right|_{\mathfrak{g}^{j}}=(-1)^{j} \mathrm{id}_{\mathfrak{g}^{j}}$ and $h \in \mathfrak{g}^{0}=\mathfrak{g}^{\tau}$ defines the grading by $\mathfrak{g}^{j}=\operatorname{ker}(\operatorname{ad} h-j \mathbf{1})$. Further, (B3/4) imply that $\mathfrak{q}:=\mathfrak{g}^{-\tau}$ is generated by the cone

$$
C \cap \mathfrak{q}=C \cap\left(\mathfrak{g}_{1} \oplus \mathfrak{g}_{-1}\right)=C_{+} \oplus-C_{-}
$$

(cf. NÓO20, Lemma 3.1]).

The group $G_{\tau}:=G \rtimes\left\{\mathbf{1}, \tau_{G}\right\}$ has a natural grading and every antiunitary representation $(U, \mathcal{H})$ of $G_{\tau}$ defines a standard subspace $\mathrm{V}=\mathrm{V}_{\left(h, \tau_{G}, U\right)}$ as in (2) ([BGL02], NÓ17]). From [Ne19b, Thm. 3.4] we know that, for $C=C_{U}$,

$$
S_{\mathrm{V}}=\exp \left(C_{+}\right) G_{\mathrm{V}} \exp \left(C_{-}\right)=G_{\mathrm{V}} \exp \left(C_{+}+C_{-}\right)
$$

where $\mathbf{L}\left(G_{\mathrm{V}}\right)=\mathfrak{g}^{0}$ and $C_{+}+C_{-}$is a pointed generating cone in $\mathfrak{q}$ (see (B3) and (27)).

Remark 3.1. If $C$ has interior points, then $\Xi:=G \exp \left(i C^{0}\right)=S_{C}^{0} \subseteq S_{C}$ is a complex manifold with the antiholomorphic involution

$$
\tau_{S}(g \exp (i x))=\tau_{G}(g) \exp (-i \tau(x))
$$

and the fixed point set

$$
S^{c}:=G^{\tau_{G}} \exp \left(i\left(C^{0} \cap \mathfrak{q}\right)\right) .
$$

Since $C$ is invariant under $e^{\mathbb{R} \text { ad } h}$, we obtain for each $t \in \mathbb{R}$ a unique automorphism $\beta_{t}$ of $S_{C}$ which induces the automorphism $e^{t \text { ad } h}$ on the Lie wedge $\mathbf{L}\left(S_{C}\right)$.

Now $S_{\mathrm{v}}^{0}$ plays the role of a "wedge domain" in the "Shilov boundary" $G$ of $S_{C}^{0}$, on which we may "realize" the standard subspace $\mathrm{V}$ as $\mathrm{H}_{\mathrm{E}}\left(S_{\mathrm{V}}^{0}\right) \subseteq \mathcal{H}_{\mathrm{E}}(G)$ for some $\mathrm{E} \subseteq \mathcal{H}^{-\infty}$.

(c) If $C$ is not generating in $\mathfrak{g}$ and $\mathfrak{g}=\mathfrak{g}_{C}+\mathbb{R} h$, then $\Xi:=G_{C} \exp \left(i C^{0}\right)$ (the open complex Olshanski semigroup associated to the pointed generating invariant cone $C \subseteq \mathfrak{g}_{C}$ ) is a complex manifold with an $\mathbb{R}^{\times}$-action and we shall realize the representation in holomorphic functions on $\Xi$, with boundary value maps to $G_{C}$. In this case $S_{\mathrm{V}}=\left(S_{\mathrm{v}} \cap G_{C}\right) A$ and we have the continuous inclusion

$$
\mathcal{H}^{\infty}(U) \hookrightarrow \mathcal{H}^{\infty}\left(\left.U\right|_{G_{C}}\right)
$$

which dualizes by Lemma A.2(b) in the appendix to a linear injection

$$
\mathcal{H}^{-\infty}\left(\left.U\right|_{G_{C}}\right)=\mathrm{d} U^{-\infty}\left(\mathcal{U}\left(\mathfrak{g}_{C}\right)\right) \mathcal{H} \hookrightarrow \mathrm{d} U^{-\infty}(\mathcal{U}(\mathfrak{g})) \mathcal{H}=\mathcal{H}^{-\infty}(U),
$$

and we have to work with elements $\eta \in \mathcal{H}^{-\infty}\left(\left.U\right|_{G_{C}}\right)$. 


\subsection{Distribution vectors associated to standard subspaces}

In this subsection we develop some results concerning the connection between standard subspaces and distribution vectors. We shall assume (B1-4), that $(U, \mathcal{H})$ is an antiunitary representation with $C=C_{U}$ pointed and that $\mathrm{V} \subseteq \mathcal{H}$ is the standard subspace specified by the triple $\left(h, \tau_{G}, U\right)$. For later reference, we note that (6) implies that, for the symplectic orthogonal space $\mathrm{V}^{\prime}$ (see (20) ), which is the standard subspace specified by the triple $\left(-h, \tau_{G}, U\right)$, the endomorphism semigroup (cf. (28)) is given by

$$
S_{\mathrm{V}^{\prime}}=S_{\mathrm{V}}^{-1} \text {. }
$$

Definition 3.2. We write $\mathrm{V}^{\infty}:=\mathrm{V} \cap \mathcal{H}^{\infty}$ for the subspace of smooth vectors contained in $\mathrm{V}$ and

$$
\mathrm{V}^{-\infty}:=\left\{\alpha \in \mathcal{H}^{-\infty}: \operatorname{Im} \alpha\left(\left(\mathrm{V}^{\prime}\right)^{\infty}\right)=\{0\}\right\}
$$

where $\mathrm{V}^{\prime}=\mathrm{V}^{\perp \omega}=\mathrm{V}_{\left(-h, \tau_{G}, U\right)}$ is the symplectic orthogonal space of $\mathrm{V}$ (see (20)).

Lemma 3.3. The following assertions hold:

(a) $\mathrm{V}^{\infty}$ is dense in $\mathrm{V}$ and $\left(\mathrm{V}^{\prime}\right)^{\infty}$ is dense in $\mathrm{V}^{\prime}$.

(b) $\mathrm{V}^{-\infty}$ is the weak-*-closure of the real subspace $\mathrm{V}$ of $\mathcal{H}^{-\infty}$.

(c) $\mathrm{V}^{-\infty}=\left\{\alpha \in \mathcal{H}^{-\infty}:\left(\forall \varphi \in C_{c}^{\infty}\left(S_{\mathrm{V}}^{0}, \mathbb{R}\right)\right) U^{-\infty}(\varphi) \alpha \in \mathrm{V}\right\}$.

(d) $\mathrm{V}^{-\infty} \cap i \mathrm{~V}^{-\infty}=\{0\}$.

(e) $\mathrm{V}^{\infty}$ is $\mathrm{d} U(\mathfrak{g})$-invariant.

(f) $\mathrm{V}^{-\infty}$ is $\mathrm{d} U^{-\infty}(\mathfrak{g})$-invariant.

Proof. (a) Let $\left(\delta_{n}\right)_{n \in \mathbb{N}}$ be a $\delta$-sequence in $C_{c}^{\infty}\left(S_{\mathrm{V}}^{0}, \mathbb{R}\right)$. For $\xi \in \mathrm{V}$, the sequence $U\left(\delta_{n}\right) \xi$ of smooth vectors is contained in $\mathrm{V}$ and converges to $\xi$. We likewise conclude that $\left(\mathrm{V}^{\prime}\right)^{\infty}$ is dense in $\mathrm{V}^{\prime}$.

(b) An element $\xi \in \mathcal{H}^{\infty}$ annihilates the subspace $\mathrm{V}$ of $\mathcal{H}^{-\infty}$ under the real bilinear pairing

$$
\mathcal{H}^{-\infty} \times \mathcal{H}^{\infty} \rightarrow \mathbb{R}, \quad(\alpha, \xi) \mapsto \operatorname{Re} \alpha(\xi)
$$

if and only if $\operatorname{Re}\langle\xi, \mathrm{V}\rangle=\{0\}$, which is equivalent to $\xi \in\left(i \mathrm{~V}^{\prime}\right) \cap \mathcal{H}^{\infty}=i\left(\mathrm{~V}^{\prime}\right)^{\infty}$. Therefore the weak-*-closure of $\mathrm{V}$ in $\mathcal{H}^{-\infty}$ is the annihilator of $i\left(\mathrm{~V}^{\prime}\right)^{\infty}$, which consists of all elements $\alpha$ satisfying $\{0\}=\operatorname{Re} \alpha\left(i\left(\mathrm{~V}^{\prime}\right)^{\infty}\right)$, which is equivalent to $\{0\}=\operatorname{Im} \alpha\left(\left(\mathrm{V}^{\prime}\right)^{\infty}\right)$, i.e., to $\alpha \in \mathrm{V}^{-\infty}$.

(c) If $\alpha \in \mathrm{V}^{-\infty}$ and $\varphi \in C_{c}^{\infty}\left(S_{\mathrm{V}}^{0}, \mathbb{R}\right)$, then any $\xi \in\left(\mathrm{V}^{\prime}\right)^{\infty}$ satisfies

$$
\operatorname{Im}\left(U^{-\infty}(\varphi) \alpha\right)(\xi)=\int_{G} \varphi(g) \operatorname{Im}\left(U^{-\infty}(g) \alpha\right)(\xi) d g=\int_{G} \varphi(g) \operatorname{Im} \alpha\left(U\left(g^{-1}\right) \xi\right) d g=0
$$

because, for $\varphi(g) \neq 0$, the relation $U\left(g^{-1}\right) \xi \in\left(\mathrm{V}^{\prime}\right)^{\infty}$ follows from $S_{\mathrm{V}^{\prime}}=S_{\mathrm{V}}^{-1}$ (see (30)).

Suppose, conversely, that $\alpha \in \mathcal{H}^{-\infty}$ is such that, for any $\varphi \in C_{c}^{\infty}\left(S_{\mathrm{V}}^{0}, \mathbb{R}\right)$ the vector $U^{-\infty}(\varphi) \alpha$ is contained in V. As $S_{\mathrm{V}}$ has dense interior ( Ne19, Thm. 4.4]), $C_{c}^{\infty}\left(S_{\mathrm{V}}^{0}, \mathbb{R}\right)$ contains a $\delta$-sequence $\left(\delta_{n}\right)_{n \in \mathbb{N}}$. For $\xi \in\left(\mathrm{V}^{\prime}\right)^{\infty}$, we then obtain

$$
\operatorname{Im} \alpha(\xi)=\lim _{n \rightarrow \infty} \operatorname{Im}\left(U^{-\infty}\left(\delta_{n}\right) \alpha\right)(\xi)=\lim _{n \rightarrow \infty} \operatorname{Im}\left\langle\xi, U^{-\infty}\left(\delta_{n}\right) \alpha\right\rangle=0 .
$$

This means that $\alpha \in \mathrm{V}^{-\infty}$.

(d) A distribution vector $\alpha$ is contained in $\mathrm{V}^{-\infty} \cap i \mathrm{~V}^{-\infty}$ if and only if $\left(\mathrm{V}^{\prime}\right)^{\infty}=\mathrm{V}^{\prime} \cap \mathcal{H}^{\infty} \subseteq$ ker $\alpha$. Let $\xi \in \mathrm{V}^{\prime}$ and $\left(\delta_{n}\right)_{n \in \mathbb{N}}$ be a $\delta$-sequence of test functions supported in the interior of $S_{\mathrm{V}^{\prime}}=\tau_{G}\left(S_{\mathrm{V}}\right)=S_{\mathrm{V}}^{-1}$. Then $U\left(\delta_{n}\right) \xi \in\left(\mathrm{V}^{\prime}\right)^{\infty} \subseteq$ ker $\alpha$ implies that $U\left(\delta_{n}\right)\left(\mathrm{V}^{\prime}+i \mathrm{~V}^{\prime}\right) \subseteq$ ker $\alpha$, and since $U\left(\delta_{n}\right): \mathcal{H} \rightarrow \mathcal{H}^{\infty}$ is continuous and $\mathrm{V}^{\prime}$ is standard, it follows that $U\left(\delta_{n}\right) \mathcal{H} \subseteq$ ker $\alpha$. Now the assertion follows from $U\left(\delta_{n}\right) v \rightarrow v$ in $\mathcal{H}^{\infty}$ for every smooth vector $v$, a consequence of the continuity of the $G$-action on the Fréchet space $\mathcal{H}^{\infty}$ (cf. [Ne10, Thm. 4.4]). 
(e) Let $\xi \in \mathrm{V}^{\infty}$ and $x \in \mathfrak{g}$. If $\left(\delta_{n}\right)_{n \in \mathbb{N}}$ is a $\delta$-sequence in $C_{c}^{\infty}\left(S_{\mathrm{V}}^{0}\right)$, then we have already observed under (d) that $U\left(\delta_{n}\right) \xi \rightarrow \xi$ in the topology of $\mathcal{H}^{\infty}$. As $\mathrm{d} U(x) U\left(\delta_{n}\right) \xi=U\left(x^{R} \delta_{n}\right) \xi \in \mathrm{V}$ by (84) in Appendix $\mathrm{A}$, passing to the limit $n \rightarrow \infty$ implies $\mathrm{d} U(x) \xi \in \mathrm{V}^{\infty}$.

(f) For $\alpha \in \mathrm{V}^{-\infty}, \xi \in\left(\mathrm{V}^{\prime}\right)^{\infty}$ and $x \in \mathfrak{g}$, we have

$$
\left(\mathrm{d} U^{-\infty}(x) \alpha\right)(\xi)=-\alpha(\mathrm{d} U(x) \xi) \in \mathbb{R}
$$

because (e), applied to the standard subspace $\mathrm{V}^{\prime}$, implies that $\mathrm{d} U(x) \xi \in\left(\mathrm{V}^{\prime}\right)^{\infty}$.

Since we shall use it several times below, we recall the following important fact on standard subspaces from [Lo08, Prop. 3.10]:

Lemma 3.4. Suppose that $\mathrm{H}_{1} \subseteq \mathrm{H}_{2}$ are standard subspaces of $\mathcal{H}$. If

(a) $\Delta_{\mathrm{H}_{2}}^{i t} \mathrm{H}_{1}=\mathrm{H}_{1}$ for every $t \in \mathbb{R}$, or

(b) $\Delta_{\mathrm{H}_{1}}^{i t} \mathrm{H}_{2}=\mathrm{H}_{2}$ for every $t \in \mathbb{R}$,

then $\mathrm{H}_{1}=\mathrm{H}_{2}$.

Proof. That (a) implies $\mathrm{H}_{1}=\mathrm{H}_{2}$ follows from [Lo08, Prop. 3.10]). From (b) we obtain by dualization $\mathrm{H}_{2}^{\prime} \subseteq \mathrm{H}_{1}^{\prime}$ with $\Delta_{\mathrm{H}_{1}^{\prime}}^{i t} \mathrm{H}_{2}^{\prime}=\mathrm{H}_{2}^{\prime}$ for $t \in \mathbb{R}$, so that we obtain $\mathrm{H}_{1}^{\prime}=\mathrm{H}_{2}^{\prime}$ with (a), hence $\mathrm{H}_{1}=\mathrm{H}_{2}$ also holds in this case.

The following theorem is an interesting tool to obtain nice descriptions of standard subspaces in concrete situations. Here a subtle point is that we assume $E \subseteq \mathrm{V}^{-\infty}$, but we shall see in Lemma 3.12 below how this assumption can be verified in terms of the action of $A=\exp (\mathbb{R} h)$ on $\mathcal{H}^{-\infty}$.

Theorem 3.5. For a real subspace $\mathrm{E} \subseteq \mathcal{H}^{-\infty}$ invariant under $A=\exp (\mathbb{R} h)$ and satisfying $\mathcal{H}_{\mathrm{E}}(G)=\mathcal{H}$, the following assertions hold:

(a) If $\emptyset \neq \mathcal{O} \subseteq G$ is open, then $\mathrm{H}_{\mathrm{E}}(\mathcal{O})$ is cyclic (Reeh-Schlieder property).

(b) If $\mathrm{E} \subseteq \mathrm{V}^{-\infty}$, then $\mathrm{H}_{\mathrm{E}}\left(S_{\mathrm{V}}^{0}\right)=\mathrm{V}$.

If $C=C_{U}$ has interior points, then $G=G_{C}$, and the Reeh-Schlieder property (Theorem 2.14) implies that $\mathrm{H}_{\mathrm{E}}(\mathcal{O})$ is cyclic in $\mathcal{H}_{\mathrm{E}}(G)$ for every non-empty open subset $\mathcal{O} \subseteq G$. Assertion (a) above shows that this remains true under the weaker assumption (B3).

Proof. (a) For $\alpha \in \mathrm{E}$ and $\varphi \in C_{c}^{\infty}(\mathcal{O}, \mathbb{R})$, the $\operatorname{support} \operatorname{supp}(\varphi)$ is a compact subset of $\mathcal{O}$. Hence there exists an $e$-neighborhood $V \subseteq G_{C}$ with $V \operatorname{supp}(\varphi) \subseteq \mathcal{O}$. This shows that

$$
U\left(C_{c}^{\infty}(V, \mathbb{R})\right) U^{-\infty}(\varphi) \alpha \subseteq U^{-\infty}\left(C_{c}^{\infty}(\mathcal{O}, \mathbb{R})\right) \alpha \subseteq \mathrm{H}_{\mathrm{E}}(\mathcal{O}) .
$$

Applying Theorem 2.14 to $\mathrm{E}=\mathbb{R} \eta$ for $\eta:=U^{-\infty}(\varphi) \alpha \in \mathcal{H} \subseteq \mathcal{H}^{-\infty}$ and the open subset $V \subseteq G_{C}$, we first see that $\mathrm{H}_{\eta}(V)$ is total in $\mathcal{H}_{\eta}\left(G_{C}\right)$, and thus

$$
U\left(G_{C}\right) \eta \subseteq \mathcal{H}_{\eta}\left(G_{C}\right)=\mathcal{H}_{\eta}(V) \stackrel{31}{\subseteq} \mathcal{H}_{\mathrm{E}}(\mathcal{O})
$$

This implies that $\mathcal{H}_{\mathrm{E}}(\mathcal{O})$ is $G_{C}$-invariant.

Lemma 2.10 (a) (ii) now implies that $\mathcal{H}_{\mathrm{E}}(\mathcal{O})=\mathcal{H}_{\mathrm{E}}\left(G_{C} \mathcal{O}\right)$. As $A$ is abelian and $G=G_{C} A$, the open subset $G_{C} \mathcal{O} \subseteq G$ is invariant under all inner automorphisms. Therefore the $A$-invariance of $\mathrm{E}$ implies that $\mathcal{H}_{\mathrm{E}}(\mathcal{O})$ is also $A$-invariant, hence $G$-invariant, and thus $\mathcal{H}_{\mathrm{E}}(\mathcal{O})=\mathcal{H}_{\mathrm{E}}(G)$ (Lemma 2.11).

(b) First, Lemma 3.3(c) implies that $\mathrm{H}_{\mathrm{E}}\left(S_{\mathrm{V}}^{0}\right) \subseteq \mathrm{V}$, hence that $\mathrm{H}_{\mathrm{E}}\left(S_{\mathrm{V}}^{0}\right)$ is separating. As $\mathrm{H}_{\mathrm{E}}\left(S_{\mathrm{V}}^{0}\right)$ is cyclic by (a), it is standard. The invariance of $\mathrm{E}$ under $U^{-\infty}(A)$ and the invariance of $S_{\mathrm{V}}^{0}$ under conjugation with $A$ entail that $\mathrm{H}_{\mathrm{E}}\left(S_{\mathrm{V}}^{0}\right)$ is invariant under $\Delta_{\mathrm{V}}^{i \mathbb{R}}=U(A)$ (see (80) in the appendix). Now $\mathrm{H}_{\mathrm{E}}\left(S_{\mathrm{V}}^{0}\right)=\mathrm{V}$ follows from Lemma 3.4. 
For $\mathrm{E}=\mathrm{V}$, we have $\mathcal{H}_{\mathrm{v}}(G)=\mathcal{H}$, and Theorem 3.5 (b) reduces to the tautology $\mathrm{H}_{\mathrm{v}}\left(S_{\mathrm{v}}^{0}\right)=\mathrm{v}$, so that this theorem is most interesting if $E$ is small. Actually it is finite-dimensional in many interesting situations (see Section 5). In particular, we would also like to have that $\mathrm{H}_{\mathrm{E}}\left(\left(S_{\mathrm{V}}^{0}\right)^{-1}\right)=\mathrm{H}_{\mathrm{E}}\left(S_{\mathrm{V}^{\prime}}^{0}\right) \subseteq \mathrm{V}^{\prime}$, but this requires

$$
\mathrm{E} \subseteq \mathrm{V}^{-\infty} \cap\left(\mathrm{V}^{\prime}\right)^{-\infty}
$$

by Lemma 3.3 (c). This is an interesting point because it may happen that the symplectic form is non-degenerate on $\mathrm{V}$, i.e., $\mathrm{V} \cap \mathrm{V}^{\prime}=\{0\}$, but that nevertheless the subspaces $\mathrm{V}^{-\infty}$ and $\left(\mathrm{V}^{\prime}\right)^{-\infty}$ have non-trivial intersection. As we shall see in Subsection 3.3 the irreducible antiunitary positive energy representation of $\mathrm{Aff}(\mathbb{R})$ provides an example where $\mathrm{V} \cap \mathrm{V}^{\prime}=\{0\}$ follows from the fact that $\Delta-\mathbf{1}$ is injective, but in this case $\mathrm{V}^{-\infty} \cap\left(\mathrm{V}^{\prime}\right)^{-\infty}$ may contain $J_{\mathrm{v}}$-fixed $\Delta$-eigenvectors. Note that

$$
\mathrm{V} \cap \mathrm{V}^{\prime}=\left\{\xi \in \mathcal{H}: \Delta_{\mathrm{V}} \xi=\xi=J_{\mathrm{V}} \xi\right\}=\operatorname{ker}\left(\Delta_{\mathrm{V}}-\mathbf{1}\right)^{J_{\mathrm{V}}}
$$

is contained in the 1-eigenspace of $\Delta_{\mathrm{V}}$.

\subsection{Extending orbit maps of distribution vectors}

In [NÓO20, Prop. 2.1] we have seen that an element $\xi \in \mathcal{H}$ is contained in the standard subspace $\mathrm{V}$ if and only if the orbit map

$$
\alpha^{\xi}: \mathbb{R} \rightarrow \mathcal{H}, \quad \alpha^{\xi}(t):=\Delta_{\mathrm{V}}^{-i t / 2 \pi} \xi
$$

extends to a continuous map on the closed strip $\overline{\mathcal{S}_{\pi}} \rightarrow \mathcal{H}$, holomorphic on $\mathcal{S}_{\pi}$, and satisfying $\alpha^{\xi}(\pi i)=J_{\mathrm{v}} \xi$. In this subsection we consider a similar condition for distribution vectors. This condition specifies a linear subspace $\mathcal{H}_{\text {ext }, J}^{-\infty} \subseteq \mathcal{H}^{-\infty}$. We then show that this space is invariant under $U^{-\infty}\left(S_{\mathrm{V}}^{0}\right)$ and $U^{-\infty}\left(C^{\infty}\left(S_{\mathrm{V}}^{0}\right)\right)$, which in turn leads to the important result that it is contained in $V^{-\infty}$ (Lemma 3.12).

Definition 3.6. Let $\eta \in \mathcal{H}^{-\infty}$ be a distribution vector. We say that

$$
\alpha^{\eta}: \mathbb{R} \rightarrow \mathcal{H}^{-\infty}, \quad \alpha^{\eta}(t):=U^{-\infty}(\exp t h) \eta
$$

extends if there exists a weak-*-continuous extension $\alpha^{\eta}: \overline{\mathcal{S}_{\pi}} \rightarrow \mathcal{H}^{-\infty}$ which is weak-*-holomorphic on $\mathcal{S}_{\pi}$. We write $\mathcal{H}_{\text {ext }}^{-\infty}$ for the linear subspace of distribution vectors with this property and $\mathcal{H}_{\text {ext }, J}^{-\infty}$ for the subspace of those $\eta \in \mathcal{H}_{\text {ext }}^{-\infty}$ for which the extension to $\overline{\mathcal{S}_{\pi}}$ satisfies $\alpha^{\eta}(\pi i)=J_{\mathrm{v}} \eta$.

Note that $\mathrm{V} \subseteq \mathcal{H}_{\text {ext }, J}^{-\infty}$ follows from the continuity of the inclusion $\mathcal{H} \hookrightarrow \mathcal{H}^{-\infty}$ with respect to the weak-*-topology ( NÓO20, Prop. 2.1]). To address the invariance properties of $\mathcal{H}_{\text {ext }}^{-\infty}$, we start with an abstract lemma.

Lemma 3.7. Let $X$ be a locally compact space and $f: X \rightarrow \mathcal{H}^{-\infty}$ be a weak-*-continuous map. Then the following assertions hold:

(a) $f^{\wedge}: X \times \mathcal{H}^{\infty} \rightarrow \mathbb{C}, f^{\wedge}(x, \xi):=f(x)(\xi)$, is continuous.

(b) If, in addition, $X$ is a complex manifold and $f$ is antiholomorphic, then $\overline{f^{\wedge}}$ is holomorphic.

Proof. (a) Since the assertion is local in the first argument, we may w.l.o.g. assume that $X$ is compact. Then $f(X)$ is pointwise bounded, hence equicontinuous because $\mathcal{H}^{\infty}$ is a Fréchet space and therefore barrelled ([Tr67, Thm. 33.2]). To see that $f^{\wedge}$ is continuous in $(x, \xi)$, we note that

$$
f\left(x^{\prime}\right)\left(\xi^{\prime}\right)-f(x)(\xi)=f\left(x^{\prime}\right)\left(\xi^{\prime}-\xi\right)+\left(f\left(x^{\prime}\right)-f(x)\right)(\xi) .
$$

As $f(\cdot)(\xi)$ is continuous and $f(X)$ is equicontinuous, the continuity of $f^{\wedge}$ follows.

(b) If, in addition, $X$ is a complex manifold and $f$ is antiholomorphic, then $\overline{f^{\wedge}}$ is a continuous map on the product of two complex Fréchet manifolds which is holomorphic in each argument separately. Hence the assertion follows from Hartogs' Theorem. Alternatively, one may combine GN, Prop. 1.2.8] with [GN Thm. 2.1.12] to see that $\overline{f^{\wedge}}$ is holomorphic. 
Since the semigroup $S_{C}$ is not a manifold because $C$ is a closed cone and $C$ may have empty interior, it is not at all clear what holomorphic functions with values in $S_{C}$ should be. Here is a definition.

Definition 3.8. ( $S_{C}$-valued holomorphic functions) Let

$$
q_{S}: \widetilde{S}_{C} \rightarrow \widetilde{G}_{\mathbb{C}}, \quad q_{S}(g \exp (i x))=\eta_{\widetilde{G}}(g) \exp _{\widetilde{G}_{\mathbb{C}}}(i x), \quad g \in \widetilde{G}, x \in C,
$$

where $\eta_{\widetilde{G}}: \widetilde{G} \rightarrow \widetilde{G}_{\mathbb{C}}$ is the universal homomorphism for which $\mathbf{L}\left(\eta_{\widetilde{G}}\right): \mathfrak{g} \rightarrow \mathfrak{g}_{\mathbb{C}}$ is the inclusion, and $\widetilde{G}_{\mathbb{C}}$ is a 1-connected Lie group with Lie algebra $\mathfrak{g}_{\mathbb{C}}$ (cf. Definition 2.1). If $M$ is a complex manifold, then we call a continuous map $f: M \rightarrow \widetilde{S}_{C}$ holomorphic if the composition $q_{S} \circ f: M \rightarrow \widetilde{G}_{\mathbb{C}}$ is holomorphic.

A map $f: M \rightarrow S_{C}$ is called holomorphic, if, for every 1-connected open subset $U \subseteq M$ a lift $\widetilde{f}_{U}: U \rightarrow \widetilde{S}_{C}$ of $\left.f\right|_{U}: U \rightarrow S_{C}$ (which exists if $U$ is 1-connected) is holomorphic.

Lemma 3.9. For $s \in S_{C}$, we write

$$
\beta^{s}(t)=\exp (t h) s \exp (-t h)
$$

for the orbit map under the conjugation action of the modular one-parameter group. For every $s \in S_{\mathrm{V}}$, this orbit map extends to a continuous map $\beta^{s}: \overline{\mathcal{S}_{\pi}} \rightarrow S_{C}$ on the closed strip $\overline{\mathcal{S}_{\pi}}$ which is holomorphic on $\mathcal{S}_{\pi}$ in the sense of Definition 3.8. The so obtained map

$$
\overline{\mathcal{S}_{\pi}} \times S_{\mathrm{V}} \rightarrow S_{C}, \quad(z, s) \mapsto \beta^{s}(z)
$$

is continuous. In addition, $\beta^{s}\left(\mathcal{S}_{\pi}\right) \subseteq S_{C}^{\circ}:=G \exp \left(i C^{\circ}\right)$ for $s \in S_{\mathrm{V}}^{0}$, where $C^{\circ}$ denotes the interior of $C$ in its span $\mathfrak{g}_{C}=C-C$.

Proof. For $s=g \exp \left(x_{1}+x_{-1}\right) \in S_{\mathrm{V}}$ with $g \in G_{\mathrm{V}}, x_{ \pm 1} \in C_{ \pm}$, we have

$$
\beta^{s}(z)=g \exp \left(e^{z} x_{1}+e^{-z} x_{-1}\right) .
$$

If $s \in S_{\mathrm{V}}^{0}$, i.e., $x_{ \pm 1} \in C_{ \pm}^{0}$, and $z \in \mathcal{S}_{\pi}$, then $\beta^{s}(z) \in S_{C}^{\circ}$ because, for $z=a+i b, 0<b<\pi$, we have

$$
\operatorname{Im}\left(e^{z} x_{1}+e^{-z} x_{-1}\right)=\sin (b)\left(e^{a} x_{1}-e^{-a} x_{-1}\right) \in C_{\mathfrak{q}}^{0} \subseteq C^{\circ} .
$$

In particular, we see that $\beta^{s}(z) \in S_{C}$, and (34) shows that the map (33) is continuous. It also shows that all maps $\beta^{s}: \mathcal{S}_{\pi} \rightarrow S_{C}, s \in S_{\mathrm{v}}$, are holomorphic in the sense of Definition 3.8

Lemma 3.10. The subspaces $\mathcal{H}_{\mathrm{ext}}^{-\infty}$ and $\mathcal{H}_{\mathrm{ext}, J}^{-\infty}$ are invariant under $U^{-\infty}\left(S_{\mathrm{V}}^{0}\right)$ and the algebra $U^{-\infty}\left(C_{c}^{\infty}\left(S_{\mathrm{V}}^{0}, \mathbb{R}\right)\right)$.

Proof. Step 1: $U^{-\infty}\left(S_{\mathrm{V}}^{0}\right) \mathcal{H}_{\text {ext }}^{-\infty} \subseteq \mathcal{H}_{\text {ext }}^{-\infty}$.

Let $\eta \in \mathcal{H}_{\text {ext }}^{-\infty}$. For $t \in \mathbb{R}$ and $g \in S_{\mathrm{v}}$, we have

$$
\alpha^{U^{-\infty}(g) \eta}(t)=U^{-\infty}(\exp t h) U^{-\infty}(g) \eta=U^{-\infty}\left(\beta^{g}(t)\right) U^{-\infty}(\exp t h) \eta=U^{-\infty}\left(\beta^{g}(t)\right) \alpha^{\eta}(t) .
$$

By Lemma 3.9 and Proposition 2.4 this curve extends to the function

$$
\overline{\mathcal{S}_{\pi}} \rightarrow \mathcal{H}^{-\infty}, \quad z \mapsto F(z, g):=U^{-\infty}\left(\beta^{g}(z)\right) \alpha^{\eta}(z)
$$

Claim 1: The map $F: \overline{\mathcal{S}_{\pi}} \times S_{\mathrm{V}} \rightarrow \mathcal{H}^{-\infty}, F(z, g)=U^{-\infty}\left(\beta^{g}(z)\right) \alpha^{\eta}(z)$ is weak-*-continuous. As $\alpha^{\eta}$ is weak-*-continuous on the locally compact space $\overline{\mathcal{S}_{\pi}}$, the weak-*-continuity of $F$ follows from Lemma 3.7(a) and the continuity of the maps

$$
\overline{\mathcal{S}_{\pi}} \times S_{\mathrm{V}} \rightarrow \mathcal{H}^{\infty}, \quad(z, g) \mapsto U\left(\beta^{g}(z)^{*}\right) \xi, \quad \xi \in \mathcal{H}^{\infty},
$$

which in turn follows from Lemma 2.3 and Lemma 3.9 
Claim 2: For $g \in S_{\mathrm{V}}^{0}$, the map $F(\cdot, g)$ is weak-*-holomorphic on $\mathcal{S}_{\pi}$.

As $\alpha^{\eta}$ is weak-*-holomorphic on $\mathcal{S}_{\pi}$, Claim 2 follows from Lemma 3.7(b) and the antiholomorphy of the maps

$$
\mathcal{S}_{\pi} \rightarrow \mathcal{H}^{\infty}, \quad z \mapsto U\left(\beta^{g}(z)^{*}\right) \xi=U\left(\beta^{g^{-1}}(\bar{z})\right) \xi, \quad \xi \in \mathcal{H}^{\infty}
$$

which in turn follows from Lemma 2.3 and the holomorphy of $\beta^{g}$ on $\mathcal{S}_{\pi}$.

Claims 1 and 2 imply that, for $g \in S_{\mathrm{V}}^{0}$, we have $U^{-\infty}(g) \eta \in \mathcal{H}_{\text {ext }}^{-\infty}$ with $\alpha^{U^{-\infty}(g) \eta}=F(\cdot, g)$.

Step 2: $U^{-\infty}\left(C_{c}^{\infty}\left(S_{\mathrm{V}}^{0}, \mathbb{R}\right)\right) \mathcal{H}_{\text {ext }}^{-\infty} \subseteq \mathcal{H}_{\text {ext }}^{-\infty}$.

For $\psi \in C_{c}^{\infty}\left(S_{\mathrm{V}}^{0}, \mathbb{R}\right)$, we consider the map

$$
f: \overline{\mathcal{S}_{\pi}} \rightarrow \mathcal{H}^{-\infty}, \quad f(z):=\int_{\operatorname{supp}(\psi)} \psi(g) F(z, g) d g=\int_{S_{\mathrm{V}}} \psi(g) U^{-\infty}\left(\beta^{g}(z)\right) \alpha^{\eta}(z) d g .
$$

That $f$ is weak-*-continuous follows from Claim 1 and [GN, Lemma 1.1.11]. That it is weak$*$-holomorphic on $\mathcal{S}_{\pi}$ likewise follows from Claim 2 with [GN Lemma 1.3.15]. This shows that $U^{-\infty}(\psi) \eta \in \mathcal{H}_{\text {ext }}^{-\infty}$ because $f=\alpha^{U^{-\infty}(\psi) \eta}$.

Step 3: Invariance of $\mathcal{H}_{\mathrm{ext}, J}^{-\infty}$.

We now assume that $\eta \in \mathcal{H}_{\mathrm{ext}, J}^{-\infty}$, so that $\alpha^{\eta}(\pi i)=J_{\mathrm{v}} \eta$. Then

$$
\alpha^{U^{-\infty}(g) \eta}(\pi i)=F(\pi i, g)=U^{-\infty}\left(\beta^{g}(\pi i)\right) \alpha^{\eta}(\pi i)=U^{-\infty}\left(\tau_{G}(g)\right) J_{\mathrm{V}} \eta=J_{\mathrm{V}} U^{-\infty}(g) \eta
$$

shows that $U^{-\infty}(g) \eta \in \mathcal{H}_{\text {ext }, J}^{-\infty}$. We further obtain with 36]

$$
\begin{aligned}
\alpha^{U^{-\infty}(\psi) \eta}(\pi i) & =f(\pi i)=\int_{S_{\mathrm{V}}} \psi(g) U^{-\infty}\left(\beta^{g}(\pi i)\right) \alpha^{\eta}(\pi i) d g \\
& =\int_{S_{\mathrm{V}}} \psi(g) J_{\mathrm{V}} U^{-\infty}(g) \eta d g=J_{\mathrm{V}} \int_{S_{\mathrm{V}}} \psi(g) U^{-\infty}(g) \eta d g=J_{\mathrm{V}} U^{-\infty}(\psi) \eta
\end{aligned}
$$

The following technical lemma is of key importance in this section. It provides a sufficient condition for elements $\eta \in \mathcal{H}$ to be contained in $\mathrm{V}$ in terms of a rather weak holomorphic extension requirement on the orbit map $\alpha^{\eta}$. Combined with Lemma 3.10, its helps us to construct elements $U^{-\infty}(\varphi) \eta \in \mathrm{V}$.

Lemma 3.11. Let $\mathcal{D} \subseteq \mathcal{H}$ be a dense complex subspace, so that $v \mapsto\langle\cdot, v\rangle$ injects $\mathcal{H}$ into the space $\mathcal{D}^{\sharp}$ of antilinear functionals $\mathcal{D} \rightarrow \mathbb{C}$. Let $\mathrm{V} \subseteq \mathcal{H}$ be a standard subspace for which $\mathcal{D}$ is invariant under $J_{\mathrm{V}}$ and $\Delta_{\mathrm{V}}^{i \mathbb{R}}$. Suppose that $\eta \in \mathcal{H}$ is such that the orbit map

$$
\alpha^{\eta}: \mathbb{R} \rightarrow \mathcal{D}^{\sharp}, \quad t \mapsto \eta \circ \Delta_{\mathrm{v}}^{i t / 2 \pi}
$$

extends to a map $\alpha^{\eta}: \overline{\mathcal{S}_{\pi}} \rightarrow \mathcal{D}^{\sharp}$ which is pointwise continuous, pointwise holomorphic on $\mathcal{S}_{\pi}$, and satisfies $\alpha^{\eta}(\pi i)=J_{\mathrm{v}} \eta$, for $\left(J_{\mathrm{v}} \eta\right)(\xi):=\overline{\eta\left(J_{\mathrm{v}} \xi\right)}$. Then $\langle\xi, \eta\rangle \in \mathbb{R}$ for every $\xi \in \mathcal{D} \cap \mathrm{V}^{\prime}$, and if $\mathcal{D} \cap \mathrm{V}^{\prime}$ is dense in $\mathrm{V}^{\prime}$, then $\eta \in \mathrm{V}$.

Proof. Let $\xi \in \mathcal{D} \cap \mathrm{V}^{\prime}$. By assumption, we have a continuous function

$$
f: \overline{\mathcal{S}_{\pi}} \rightarrow \mathbb{C}, \quad f(z)=\alpha^{\eta}(z)(\xi)
$$

which is holomorphic on $\mathcal{S}_{\pi}$. This function satisfies for $t \in \mathbb{R}$

$$
f(t+\pi i)=\alpha^{\eta}(t+\pi i)(\xi)=\alpha^{\eta}(\pi i)\left(\Delta_{\mathrm{V}}^{i t / 2 \pi} \xi\right)=\left(J_{\mathrm{V}} \eta\right)\left(\Delta_{\mathrm{V}}^{i t / 2 \pi} \xi\right)=\overline{\eta\left(\Delta_{\mathrm{V}}^{i t / 2 \pi} J_{\mathrm{V}} \xi\right)} .
$$

As $\xi \in \mathrm{V}^{\prime}$, we also have for $t \in \mathbb{R}$

$$
f(t)=\alpha^{\eta}(t)(\xi)=\left\langle\Delta_{\mathrm{V}}^{i t / 2 \pi} \xi, \eta\right\rangle
$$

and this function extends to a continuous function on $-\overline{\mathcal{S}_{\pi}}$, holomorphic on $-\mathcal{S}_{\pi}$, given by

$$
f(z)=\left\langle\Delta_{\mathrm{V}}^{i \bar{z} / 2 \pi} \xi, \eta\right\rangle .
$$


We thus obtain a continuous function

$$
f: \overline{\mathcal{S}_{-\pi, \pi}}=\{z \in \mathbb{C}:-\pi \leq \operatorname{Im} z \leq \pi\} \rightarrow \mathbb{C},
$$

holomorphic on the complement of the real line in the interior $\mathcal{S}_{-\pi, \pi}$, so that Morera's Theorem implies that it is holomorphic on $\mathcal{S}_{-\pi, \pi}$. On the lower boundary we have

$$
f(t-\pi i)=\left\langle\Delta_{\mathrm{V}}^{i t / 2 \pi} \Delta_{\mathrm{v}}^{-1 / 2} \xi, \eta\right\rangle=\left\langle\Delta_{\mathrm{V}}^{i t / 2 \pi} J_{\mathrm{v}} \xi, \eta\right\rangle=\eta\left(\Delta_{\mathrm{V}}^{i t / 2 \pi} J_{\mathrm{v}} \xi\right)=\overline{f(t+\pi i)} .
$$

Therefore the function

$$
F:\{z \in \mathbb{C}:-\pi \leq \operatorname{Im} z \leq \pi\} \rightarrow \mathbb{C}, \quad F(z):=f(z)-\overline{f(\bar{z})}
$$

is continuous, holomorphic on the interior, and vanishes on the line $\mathbb{R}+\pi i$. This implies that $F=0$, and evaluating on the real line shows that $f(\mathbb{R}) \subseteq \mathbb{R}$. For $t=0$, we obtain in particular $f(0)=\langle\xi, \eta\rangle \in \mathbb{R}$.

If $\mathcal{D} \cap \mathrm{V}^{\prime}$ is dense in $\mathrm{V}^{\prime}$, then this further leads to $\eta \in\left(\mathrm{V}^{\prime}\right)^{\prime}=\mathrm{V}$.

Lemma 3.12. $\mathcal{H}_{\mathrm{ext}, J}^{-\infty} \subseteq \mathrm{V}^{-\infty}$.

Proof. First we show that

$$
\mathcal{H}_{\text {ext }, J}^{-\infty} \cap \mathcal{H} \subseteq \mathrm{V} .
$$

We apply Lemma 3.11 with $\mathcal{D}=\mathcal{H}^{\infty}$ and $\eta \in \mathcal{H} \cap \mathcal{H}_{\text {ext, }}^{-\infty}$. As $\mathrm{V}^{\prime} \cap \mathcal{H}^{\infty}$ is dense in $\mathrm{V}^{\prime}$ by Lemma 3.3 (a), we obtain $\eta \in \mathrm{V}$. This proves 38.

For $\eta \in \mathcal{H}_{\text {ext }, J}^{-\infty}$ and $\psi \in C_{c}^{\infty}\left(S_{\mathrm{v}}^{0}, \mathbb{R}\right)$, Lemma 3.10 implies that $U^{-\infty}(\psi) \eta \in \mathcal{H}_{\text {ext }, J}^{-\infty}$, and since this is actually an element of $\mathcal{H}$, (38) shows that $U^{-\infty}(\psi) \eta \in \mathrm{V}$. Now the assertion follows from Lemma 3.3(c).

Proposition 3.13. Let $F \subseteq \mathcal{H}_{\mathrm{ext}, J}^{-\infty}$ be a real linear subspace which is $G$-cyclic in the sense that $\mathcal{H}_{\mathrm{F}}(G)=\mathcal{H}$, let $A=\exp (\mathbb{R} h)$, and

$$
\mathrm{E}:=\operatorname{span}_{\mathbb{R}}\left(U^{-\infty}(A) \mathrm{F}\right) \subseteq \mathcal{H}^{-\infty} .
$$

Then

$$
\mathrm{H}_{\mathrm{E}}\left(S_{\mathrm{V}}^{0}\right)=\mathrm{V}
$$

Proof. Since the subspace $\mathcal{H}_{\text {ext, }, J}^{-\infty}$ is $A$-invariant, we have $\mathrm{E} \subseteq \mathcal{H}_{\text {ext }, J}^{-\infty} \subseteq \mathrm{V}^{-\infty}$ (Lemma 3.12). As F is $G$-cyclic, $\mathcal{H}_{\mathrm{E}}(G) \supseteq \mathcal{H}_{\mathrm{F}}(G)=\mathcal{H}$, so that Theorem 3.5 (b) implies that $\mathrm{H}_{\mathrm{E}}\left(S_{\mathrm{V}}^{0}\right)=\mathrm{V}$.

Remark 3.14. (a) If $\eta \in \mathcal{H}^{-\infty}$ is contained in a finite dimensional complex $A$-invariant subspace $\mathcal{K} \subseteq \mathcal{H}^{-\infty}$, then the representation of $A$ on $\mathcal{K}$ is continuous because it is continuous on its dual space $\mathcal{H}^{\infty} / \mathcal{K}^{\perp}$. Hence it extends to a holomorphic representation $\rho$ of $A_{\mathbb{C}} \cong \mathbb{C}$ on the finite dimensional complex vector space $\mathcal{K}$, and therefore $\mathcal{K} \subseteq \mathcal{H}_{\text {ext }}^{-\infty}$.

If, in addition, $J \mathcal{K} \subseteq \mathcal{K}$ and $J_{\mathcal{K}}:=\left.J\right|_{\mathcal{K}}$, then the real subspace

$$
\mathrm{E}:=\operatorname{Fix}\left(J_{\mathcal{K}} \rho(\exp (\pi i h))\right) \subseteq \mathcal{H}_{\mathrm{ext}, J} \subseteq \mathrm{V}^{-\infty}
$$

of $\mathcal{K}$ satisfies

$$
\mathrm{H}_{\mathrm{E}}\left(S_{\mathrm{V}}^{0}\right)=\mathrm{V} \text {. }
$$

(b) Below we shall need the following more general fact. We assume that $(\rho, \mathcal{K})$ is a normcontinuous representation of $A \cong \mathbb{R}$ on the Hilbert space $\mathcal{K}$ by symmetric operators and that we have a continuous $A$-equivariant map $\eta: \mathcal{H}^{\infty} \rightarrow \mathcal{K}$ with dense range. The adjoint map $\eta^{*}: \mathcal{K} \rightarrow \mathcal{H}^{-\infty}, \eta^{*}(\xi)(v):=\langle\eta(v), \xi\rangle$ defines a weak-* continuous equivariant embedding. Since $\eta^{*}$ is $A$-equivariant and $\rho$ extends to a holomorphic representation of $A_{\mathbb{C}}$ on $\mathcal{K}$, we then have $\eta^{*}(\mathcal{K}) \subseteq \mathcal{H}_{\text {ext }}^{-\infty}$. 
Remark 3.15. We assume that $\operatorname{ker}(U)$ is discrete. Clearly, regularity of v, i.e., that $\mathbf{L}\left(S_{\mathrm{V}}\right)$ spans $\mathfrak{g}$, implies $e \in \overline{S_{\mathrm{V}}^{0}}$, but the converse is not clear. In view of the Germ Theorem ( Thm. 4.1]), $e \in \overline{S_{\mathrm{V}}^{0}}$ is equivalent to $e \in \overline{S_{C, \text { inv }}^{0}}$, where

$$
S_{C, \text { inv }}=\left\{g \in G:\left(\forall z \in \overline{\mathcal{S}_{\pi}}\right) \beta^{g}(z) \in G \exp (i C)\right\} .
$$

From [Ne19, Lemma 5.4(ii)] it follows that $e \in \overline{S_{C \text {,inv }}^{0}}$ implies that $\tau=\left.e^{\pi i \text { ad } h}\right|_{\mathfrak{g}}$, hence $e^{2 \pi i \text { ad } h}=$ $\operatorname{id}_{\mathfrak{g}_{\mathbb{C}}}$, so that $\operatorname{ad} h$ is diagonalizable with integral eigenvalues. Presently we do not know how to derive from $e \in \overline{S_{C \text {,inv }}^{0}}$ that $\mathbf{L}\left(S_{\mathrm{V}}\right)$ has interior points. The main difficulty is to show that $\operatorname{Spec}(\operatorname{ad} h) \subseteq\{-1,0,1\}$.

Although we will not need it in the following, we record the following invariance property:

Lemma 3.16. The subspaces $\mathcal{H}_{\text {ext }, J}^{-\infty} \subseteq \mathcal{H}_{\text {ext }}^{-\infty}$ are both invariant under $\mathrm{d} U^{-\infty}(\mathfrak{g})$.

Proof. If suffices to prove invariance under $\mathrm{d} U^{-\infty}\left(\mathfrak{g}^{j}\right)$ for $j=1,0,-1$. Let $\eta \in \mathcal{H}_{\text {ext }}^{-\infty}$ and $x \in \mathfrak{g}^{j}$, so that $[h, x]=j x$. For $\eta_{x}:=\mathrm{d} U^{-\infty}(x) \eta$, we then we then have

$$
\alpha^{\eta_{x}}(t)=\mathrm{d} U^{-\infty}\left(e^{t \operatorname{ad} h} x\right) \alpha^{\eta}(t)=e^{t j} \mathrm{~d} U^{-\infty}(x) \alpha^{\eta}(t) \quad \text { for } \quad t \in \mathbb{R} .
$$

Hence the map

$$
\alpha^{\eta_{x}}: \overline{\mathcal{S}_{\pi}} \rightarrow \mathcal{H}^{-\infty}, \quad z \mapsto \mathrm{d} U^{-\infty}\left(e^{z \operatorname{ad} h} x\right) \alpha^{\eta}(z)=e^{j z} \mathrm{~d} U^{-\infty}(x) \alpha^{\eta}(z)
$$

is weak-*-continuous and weak-*-holomorphic on the interior. This proves the invariance of $\mathcal{H}_{\text {ext }}^{-\infty}$. If, in addition, $\alpha^{\eta}(\pi i)=J \eta$, then we further get

$$
\alpha^{\eta_{x}}(\pi i)=\mathrm{d} U^{-\infty}\left(e^{\pi i \operatorname{ad} h} x\right) \alpha^{\eta}(\pi i)=\mathrm{d} U^{-\infty}(\tau(x)) J \eta=J \mathrm{~d} U^{-\infty}(x) \eta=J \eta_{x} .
$$

\subsection{An example: The $a x+b$-group}

We consider the affine group $G=\operatorname{Aff}(\mathbb{R})_{0}$ of the real line with

$$
G_{\tau}=\operatorname{Aff}(\mathbb{R}) \cong \mathbb{R} \rtimes \mathbb{R}^{\times} \quad \text { and } \quad \tau_{G}(b, a)=(-b, a) .
$$

The Lie algebra data is given by

$$
C=[0, \infty), \quad h:=(0,1), \quad q:=(1,0), \quad \text { and } \quad \mathfrak{g}^{0}=\mathbb{R} h, \quad \mathfrak{g}^{1}=\mathbb{R} q .
$$

Proposition 3.17. For the irreducible antiunitary $C$-positive representation $(U, \mathcal{H})$ of $\operatorname{Aff}(\mathbb{R})$, given by

$$
\mathcal{H}=L^{2}\left(\mathbb{R}_{+}^{\times}, \frac{d p}{p}\right) \quad \text { and } \quad\left(U\left(b, e^{t}\right) f\right)(p)=e^{i b p} f\left(e^{t} p\right), \quad U(0,-1) f=\bar{f},
$$

the following assertions hold:

(a) The power functions $\left(p^{s}\right)_{\operatorname{Re} s>0}$ define distribution vectors

$$
\eta_{s}(f):=\int_{\mathbb{R}^{\times}} \overline{f(p)} p^{s} \frac{d p}{p}
$$

for the restriction $U_{N}$ to the translation group $N=\mathbb{R} \times\{1\}$.

(b) These distribution vectors transform under the action of the dilation group $\mathbb{R}^{\times}$by

$$
U^{-\infty}(0, a) \eta_{s}=a^{s} \eta_{s} \quad \text { for } \quad a>0 \quad \text { and } \quad U^{-\infty}(0,-1) \eta_{s}=\eta_{\bar{s}}
$$

(c) The distribution $D_{s}:=j_{\eta_{s}}\left(\eta_{s}\right) \in \mathcal{S}^{\prime}(\mathbb{R})$ (cf. (86) in Appendix (A) coincides with the Fourier transform of the measure $p^{2 \operatorname{Re} s-1} d p$ on $\mathbb{R}_{+}$. 
Proof. (a) As $\mathcal{H}^{-\infty}\left(U_{N}\right)=\operatorname{span}_{\mathbb{C}}\left(\mathrm{d} U^{-\infty}(\mathcal{U}(\mathbb{R} q)) \mathcal{H}\right)$ (Lemma A.2), it suffices to show that there exists a polynomial $F(p) \in \mathbb{C}[p]$ with $F(p)^{-1} p^{s} \in L^{2}\left(\mathbb{R}_{+}^{\times}, \frac{d p}{p}\right)$. For $F(p):=\left(1+p^{2}\right)^{n}$, we have

$$
\left\|F(p)^{-1} p_{s}\right\|_{2}^{2}=\int_{0}^{\infty} \frac{p^{2 \operatorname{Re} s}}{\left(1+p^{2}\right)^{2 n}} \frac{d p}{p},
$$

and this integral is finite if and only if $\operatorname{Re} s>0$ and $2 n>\operatorname{Re} s$.

(b) Next we note that

$$
\left(U^{-\infty}\left(0, e^{t}\right) \eta_{s}\right)(f)=\eta_{s}\left(U\left(0, e^{-t}\right) f\right)=\int_{\mathbb{R}^{\times}} \overline{f\left(e^{-t} p\right)} p^{s} \frac{d p}{p}=\int_{\mathbb{R}^{\times}} \overline{f(p)}\left(e^{t} p\right)^{s} \frac{d p}{p}=e^{t s} \eta_{s}(f)
$$

and

$$
\left(U^{-\infty}(0,-1) \eta_{s}\right)(f)=\overline{\eta_{s}(\bar{f})}=\int_{\mathbb{R}^{\times}} \overline{f(p)} p^{\bar{s}} \frac{d p}{p}=\eta_{\bar{s}}(f),
$$

so that $\eta_{s} \in \mathcal{H}^{-\infty}$ is an $\exp (\mathbb{R} h)$-eigenvector satisfying (39).

(c) For $\varphi \in C_{c}^{\infty}(\mathbb{R}, \mathbb{R})$, we have

$$
\left(U^{-\infty}(\varphi) \eta_{s}\right)(p)=\widetilde{\varphi}(p) p^{s}, \quad \widetilde{\varphi}(p)=\int_{\mathbb{R}} e^{i p x} \varphi(x) d x
$$

because

$$
\eta_{s}\left(U\left(\varphi^{*}\right) f\right)=\int_{0}^{\infty} p^{s} \overline{\widetilde{\varphi^{*}}(p)} \overline{f(p)} \frac{d p}{p}=\int_{0}^{\infty} p^{s} \widetilde{\varphi}(p) \overline{f(p)} \frac{d p}{p} .
$$

For the distribution $D_{s}=j_{\eta_{s}}\left(\eta_{s}\right) \in C^{-\infty}(\mathbb{R})$, we thus obtain

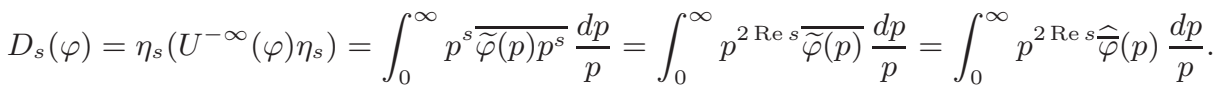

This is (c).

To identify corresponding standard subspaces, we note that $\alpha^{\eta_{s}}(z)=e^{s z} \eta_{s}$ implies that $\alpha^{\eta_{s}}(\pi i)=e^{\pi i s} \eta_{s}$ and $J \eta_{s}=\eta_{\bar{s}}$. For $s \in \mathbb{R}$, it follows that

$$
\widetilde{\eta}_{s}:=e^{-\frac{s \pi i}{2}} \eta_{s} \in \mathcal{H}_{\text {ext }, J}^{-\infty} \subseteq \mathrm{V}^{-\infty} .
$$

Accordingly, $\mathrm{v} \subseteq \mathcal{H}$ is generated by the functions

$$
\widetilde{\varphi} \widetilde{\eta}_{s}=e^{-\frac{s \pi i}{2}} \widetilde{\varphi} \eta_{s} \quad \text { for } \quad \varphi \in C_{c}^{\infty}((0, \infty), \mathbb{R}) .
$$

For $s \notin \mathbb{R}$, we put

$$
\widetilde{\eta}_{s}:=\eta_{s}+e^{-\pi i \bar{s}} \eta_{\bar{s}}
$$

and obtain $J \widetilde{\eta}_{s}=e^{\pi i s} \eta_{s}+\eta_{s}=\alpha^{\widetilde{\eta}_{s}}(\pi i)$, so that

$$
\widetilde{\eta}_{s} \in \mathcal{H}_{\mathrm{ext}, J}^{-\infty} \subseteq \mathrm{V}^{-\infty} .
$$

\section{Realizing $C$-positive representations on tubes}

In this section we show that, for semisimple Lie algebras $\mathfrak{g}$, which are direct sums of simple hermitian ideals of tube type, and any pointed generating invariant cone $C \subseteq \mathfrak{g}$, the (irreducible) $C$-positive unitary representation can be realized in Hilbert spaces of holomorphic functions on a tube domain. This realization will be used in Section 5 to exhibit a suitable real subspace $\mathrm{E} \subseteq \mathcal{H}_{\mathrm{ext}, J}^{-\infty}$ to which the results from Section 3 apply. 
Let $\mathfrak{g}$ be a semisimple Lie algebra and $h \in \mathfrak{g}$ be an Euler element for which $\mathfrak{h}:=\mathfrak{g}^{0}(h)$ contains no non-zero ideal of $\mathfrak{g}$, i.e., the Lie algebra $\mathfrak{g}$ is generated by $\mathfrak{g}^{ \pm 1}=\mathfrak{g}^{ \pm 1}(h)$. We consider the Lie groups

$$
G:=\operatorname{Inn}(\mathfrak{g})=\operatorname{Aut}(\mathfrak{g})_{0} \quad \text { and } \quad G_{\tau}:=G \rtimes\{\mathbf{1}, \tau\} \quad \text { for } \quad \tau=\left.e^{\pi i \text { ad } h}\right|_{\mathfrak{g}} .
$$

Then $G_{\tau}$ is a graded Lie group and we put $H:=\operatorname{Inn}_{\mathfrak{g}}(\mathfrak{h})=\left(G^{\tau}\right)_{0}$. We also fix a Cartan involution $\theta$ of $\mathfrak{g}$ fixing the center pointwise and satisfying $\theta(h)=-h$. The corresponding Cartan decomposition is written $\mathfrak{g}=\mathfrak{k} \oplus \mathfrak{p}$, and we write $K:=\langle\exp \mathfrak{k}\rangle$ for the corresponding maximal compact subgroup of $G$.

We further assume that all simple ideals of $\mathfrak{g}$ are hermitian, so that the existence of an Euler element implies that they are tube type (cf. [MN20, Thm. 3.12]; Ó91, Thm. 5.6]) and that we may consider the eigenspace $E:=\mathfrak{g}^{1}(h)$ as a, not necessarily simple, unital euclidean Jordan algebra. We may further pick the unit element $e \in E$ in such a way that it is fixed by $H_{K}=H^{\theta}$. Then

$$
H_{K} \cong \operatorname{Aut}(E, e)_{0} \quad \text { and } \quad H \cong \operatorname{Str}(E)_{0} \subseteq H_{\mathbb{C}}:=\operatorname{Str}\left(E_{\mathbb{C}}\right)_{0}
$$

are the identity components of the real and complex structure groups. Accordingly, we identify them with linear groups acting on $E_{\mathbb{C}}$. We then have the two associated complex domains:

- the tube domain $\mathcal{T}:=\mathcal{T}_{E_{+}}:=E+i E_{+} \subseteq E_{\mathbb{C}}$, and

- the unit ball $\mathcal{D}:=\left\{z \in E_{\mathbb{C}}:\|z\|_{\infty}<1\right\}$, with respect to the spectral norm $\|\cdot\|_{\infty}$ of $E_{\mathbb{C}}$, which is a bounded symmetric domain

The Cayley transform

$$
p: \mathcal{T} \rightarrow \mathcal{D}, \quad p(z):=(z-i e)(z+i e)^{-1}
$$

maps the tube $\mathcal{T}$ biholomorphically onto $\mathcal{D}$. Its inverse is given by

$$
c: \mathcal{D} \rightarrow \mathcal{T}, \quad c(z):=i(e+z)(e-z)^{-1}
$$

(cf. FK94, Thm. X.4.3]). The differential of the Cayley transform is given by

$$
\mathrm{d} p(z)=2 i P(z+i e)^{-1} \quad \text { for } \quad z \in \mathcal{T}
$$

where

$$
P: E_{\mathbb{C}} \rightarrow \operatorname{End}\left(E_{\mathbb{C}}\right), \quad P(z):=2 L(z)^{2}-L\left(z^{2}\right), \quad L(z) w:=z w,
$$

is the quadratic representation ([FK94, p. 192]). We have $P\left(E_{\mathbb{C}}^{\times}\right) \subseteq \operatorname{Str}\left(E_{\mathbb{C}}\right)$ ([FK94 Prop. VIII.2.4]), hence in particular

$$
P(\mathcal{T}) \subseteq H_{\mathbb{C}} \quad \text { because } \quad P(i e)=-P(e)=-\mathbf{1} \in \mathbb{C}^{\times} \mathbf{1}=\exp (\mathbb{C} h) \subseteq H_{\mathbb{C}}
$$

(cf. [FK94, p. 168]).

The group $G \cong \operatorname{Conf}(E)_{0}$ is generated by the subgroups $N^{+}:=\exp \left(\mathfrak{g}^{1}(h)\right) \cong(E,+)$ (the translation group of $E$ ), the subgroup $H$ and an element $\eta \in Z(K)$ acting on $E_{\mathbb{C}}^{\times}$by the involution

$$
\eta(z)=-z^{-1} \quad \text { with differential } \quad \mathrm{d} \eta(z)=P(z)^{-1}
$$

([FK94 Prop. II.3.3(i)]). This is the point symmetry in the point ie of the Riemannian symmetric space $\mathcal{T}$. The element $\eta \in G$ implements the Cartan involution $\theta$ of $G$ by

$$
\theta(g)=\eta \circ g \circ \eta^{-1}=\eta \circ g \circ \eta .
$$

We write $G_{\mathbb{C}}:=\operatorname{Aut}\left(\mathfrak{g}_{\mathbb{C}}\right)_{0}$ and observe that $H_{\mathbb{C}}=\left\langle\exp \mathfrak{h}_{\mathbb{C}}\right\rangle$. For $g \in H$, we put

$$
g^{*}:=\theta(g)^{-1},
$$


and if $\bar{\theta}$ denotes the antiholomorphic involution of $H_{\mathbb{C}}$ extending $\theta$, we likewise put

$$
g^{*}:=\bar{\theta}(g)^{-1} \quad \text { for } \quad g \in H_{\mathbb{C}} .
$$

We also use this notation for elements of the simply connected covering group $q_{H_{\mathbb{C}}}: \widetilde{H}_{\mathbb{C}} \rightarrow H_{\mathbb{C}}$ and the lift of $\bar{\theta}$ to $\widetilde{H}_{\mathbb{C}}$. We note that the kernel of $q_{H_{\mathbb{C}}}$ is a discrete central subgroup. As $\mathcal{T}$ is simply connected, there exists a holomorphic lift

$$
\widetilde{P}: \mathcal{T} \rightarrow \widetilde{H}_{\mathbb{C}}
$$

As $P(i e)=\mathbf{- 1}$, any such lift is determined by $\widetilde{P}(i e) \in q_{H_{\mathbb{C}}}^{-1}(-\mathbf{1})$, which is a discrete subgroup of the center of $\widetilde{H}_{\mathbb{C}}$; a coset of $\operatorname{ker}\left(q_{H_{\mathbb{C}}}\right)$. We also observe that $\widetilde{P}(i e)$ is contained in the connected subgroup $\left(\widetilde{H}_{\mathbb{C}}\right)^{\bar{\theta}}$ of unitary elements with respect to the involution $g^{*}=\bar{\theta}(g)^{-1}$.

We write $\tau_{H_{\mathbb{C}}}(g)=\bar{g}=\tau g \tau$ for the antiholomorphic automorphism of $H_{\mathbb{C}}$ induced by complex conjugation on $E_{\mathbb{C}}$. Its lift to $\widetilde{H}_{\mathbb{C}}$ is denoted $\tau_{\widetilde{H}_{\mathbb{C}}}$. We write $H^{\sharp}=\left(\widetilde{H}_{\mathbb{C}}\right)^{\tau_{\widetilde{H}_{\mathbb{C}}} \text { for its }}$ connected group of fixed points ([Lo69, Thm. IV.3.4]).

\subsection{Translating between tube and ball}

Let $G(\mathcal{D})=\operatorname{Aut}(\mathcal{D})_{0}$ be the identity component of the group of holomorphic automorphisms of $\mathcal{D}$ and $G(\mathcal{T})=\operatorname{Aut}(\mathcal{T})_{0} \cong G$ be the corresponding group for the tube domain $\mathcal{T}$. Both are subgroups of the conformal group $\operatorname{Conf}\left(E_{\mathbb{C}}\right)$, and in this group they are conjugate by the Cayley transform

$$
G(\mathcal{D})=c^{-1} G(\mathcal{T}) c \subseteq G_{\mathbb{C}}
$$

For the simply connected covering groups $q_{G(\mathcal{D})}: \widetilde{G}(\mathcal{D}) \rightarrow G(\mathcal{D})$ and $q_{G(\mathcal{T})}: \widetilde{G}(\mathcal{T}) \rightarrow G(\mathcal{T})$ we define action and $\mathcal{D}$ and $\mathcal{T}$, respectively, by $g . z:=\alpha_{G(\mathcal{D})}(g)(z)$ and $g . z:=\alpha_{G(\mathcal{T})}(g)(z)$.

Let

$$
\widetilde{\alpha}_{c}: \widetilde{G}(\mathcal{D}) \rightarrow \widetilde{G}(\mathcal{T})
$$

be the isomorphism obtained by lifting the isomorphism

$$
\alpha_{c}: G(\mathcal{D}) \rightarrow G(\mathcal{T}), \quad \alpha_{c}(g):=c g c^{-1} .
$$

We write

$$
K(\mathcal{D}):=G(\mathcal{D})^{0} \quad \text { and } \quad K(\tau):=G(\mathcal{T})^{i e}
$$

for the stabilizer groups of the base points $0 \in \mathcal{D}$ and $i e \in \mathcal{T}$.

On the tube domain $\mathcal{T}=E+i E_{+}$, we consider the universal kernel

$$
Q: \mathcal{T} \times \mathcal{T} \rightarrow H_{\mathbb{C}}, \quad Q(z, w):=P\left(\frac{z-\bar{w}}{2 i}\right),
$$

which satisfies $Q(i e, i e)=P(e)=\mathbf{1}$. It is sesquiholomorphic in the sense that it is holomorphic in the first and antiholomorphic in the second argument. As $\mathcal{T}$ is simply connected, this kernel lifts to a sesquiholomorphic kernel

$$
\widetilde{Q}: \mathcal{T} \times \mathcal{T} \rightarrow \widetilde{H}_{\mathbb{C}} \quad \text { with } \quad \widetilde{Q}(i e, i e)=\mathbf{1} .
$$

The action of $G(\mathcal{T})$ on $\mathcal{T}$ defines a cocycle

$$
J: G(\mathcal{T}) \times \mathcal{T} \rightarrow H_{\mathbb{C}}, \quad J(g, z):=J_{g}(z):=\mathrm{d} \sigma_{g}(z)
$$

satisfying

$$
J\left(g_{1} g_{2}, z\right)=J\left(g_{1}, g_{2} . z\right) J\left(g_{2}, z\right) \quad \text { for } \quad g \in G, z \in \mathcal{T} .
$$

Since $\mathcal{T}$ is simply connected, this cocycle lifts to a smooth cocycle

$$
\widetilde{J}: \widetilde{G}(\mathcal{T}) \times \mathcal{T} \rightarrow \widetilde{H}_{\mathbb{C}} \quad \text { determined by } \quad \widetilde{J}(e, i e)=\mathbf{1},
$$

which is holomorphic in the second argument. The cocycle property of $\widetilde{J}$ follows by the uniqueness of lifts, which immediately implies $\widetilde{J}(e, z)=\mathbf{1}$ for every $z \in \mathcal{T}$. We have 
- $\widetilde{J}_{g}=1$ for translations $g(x)=x+v, \in E$,

- On the connected subgroup $\widetilde{H}(\mathcal{T})=\langle\exp \mathfrak{h}\rangle \subseteq \widetilde{G}(\mathcal{T})$, the cocycle $\widetilde{J}$ defines a morphism of Lie groups $\left.\widetilde{J}\right|_{\widetilde{H}(\mathcal{T})}: \widetilde{H}(\mathcal{T}) \rightarrow H^{\sharp} \subseteq \widetilde{H}_{\mathbb{C}}$ integrating the inclusion $\mathfrak{h} \hookrightarrow \mathfrak{h}_{\mathbb{C}}$.

Lemma 4.1.

$$
\widetilde{Q}(g . z, g . w)=\widetilde{J}(g, z) \widetilde{Q}(z, w) \widetilde{J}(g, w)^{*} \quad \text { for } \quad z \in \mathcal{T}, g \in \widetilde{G}(\mathcal{T}) .
$$

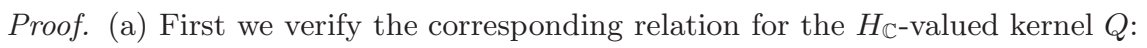

$$
Q(g . z, g . w)=J(g, z) Q(z, w) J(g, w)^{*} \quad \text { for } \quad z \in \mathcal{T}, g \in \widetilde{G}(\mathcal{T}) .
$$

Since $G=G(\mathcal{T})$ is generated by $H, \eta$ and translations and $J$ is a cocycle, we have to verify this relation only for these three cases.

For $g \in H$ we have

$$
Q(g . z, g . w)=P\left(\frac{g \cdot z-\bar{g} \cdot \bar{w}}{2 i}\right)=P\left(g \cdot \frac{z-\bar{w}}{2 i}\right) \stackrel{!}{=} g P\left(\frac{z-\bar{w}}{2 i}\right) g^{*}=J(g, z) Q(z, w) J(g, w)^{*},
$$

where we use [FK94, p. 147] for !. For $u \in E$ and the translation $g=\exp u \in N^{+}$, we obtain

$$
Q(z+u, w+u)=P\left(\frac{z+u-\bar{w}-\bar{u}}{2 i}\right)=P\left(\frac{z-\bar{w}}{2 i}\right)=Q(z, w)=J(g, z) Q(z, w) J(g, w)^{*}
$$

because $J(g, z)=1$. Finally we see with [FK94, Lemma X.4.4(i)] that

$$
\begin{aligned}
Q\left(-z^{-1},-w^{-1}\right) & =P\left(\frac{-z^{-1}+\bar{w}^{-1}}{2 i}\right)=-\frac{1}{4} P\left(-z^{-1}+\bar{w}^{-1}\right) \\
& =-\frac{1}{4} P(-z)^{-1} P(-z+\bar{w}) P(\bar{w})^{-1}=P(z)^{-1} P\left(\frac{z-\bar{w}}{2 i}\right) P(\bar{w})^{-1} \\
& =J(\eta, z) Q(z, w) J(\eta, w)^{*}
\end{aligned}
$$

because $P(w)^{*}=\overline{P(w)}=P(\bar{w})$ and $J(\eta, z)=P(z)^{-1}$.

(b) Now we turn to the lifted kernel $\widetilde{Q}$. Fix $z, w \in \mathcal{T}$. Then both sides of (46) take the same values in $g=e$. By (a), they are lifts of the same maps to $H_{\mathbb{C}}$. Hence the assertion follows from the uniqueness of continuous lifts.

Remark 4.2. The preceding lemma has some remarkable consequences.

(a) For $g \in H$, we have

$$
Q(g . i e, g . i e)=g g^{*},
$$

which is the quadratic representation of the Riemannian symmetric space $E_{+} \cong i E_{+} \subseteq \mathcal{T}$ with values in its isometry group $(H, \theta)$.

(b) For elements of the stabilizer group $\widetilde{K}=\widetilde{K}(\mathcal{T})=\widetilde{G}(\mathcal{T})^{i e}$, we have

$$
\widetilde{J}(g, i e) \widetilde{J}(g, i e)^{*}=\mathbf{1} \quad \text { for } \quad g \in \widetilde{K},
$$

so that $\widetilde{J}$ defines a homomorphism

$$
\widetilde{J}: \widetilde{K} \rightarrow\left(\widetilde{H}_{\mathbb{C}}\right)^{\bar{\theta}}=\widetilde{K}(\mathcal{D}) .
$$

Using the Cayley transform, we obtain a kernel on $\mathcal{D}$ by

$$
\begin{aligned}
Q^{\mathcal{D}}(z, w) & :=4 \cdot \mathrm{d} c(z)^{-1} Q(c(z), c(w))\left(\mathrm{d} c(w)^{-1}\right)^{*} \\
& =4 \cdot \mathrm{d} p(c(z)) Q(c(z), c(w))(\mathrm{d} p(c(w)))^{*} \\
& \stackrel{40}{=} P\left(\frac{c(z)+i e}{2}\right)^{-1} Q(c(z), c(w))\left(P\left(\frac{c(z)+i e}{2}\right)^{-1}\right)^{*} .
\end{aligned}
$$


The kernel $Q^{\mathcal{D}}$ also takes values in $H_{\mathbb{C}}$, and $c(0)=i e$ leads in particular to

$$
Q^{\mathcal{D}}(0,0)=P(i e)^{-1} Q(i e, i e)\left(P(i e)^{-1}\right)^{*}=\mathbf{1},
$$

so that there exists a unique continuous lift

$$
\widetilde{Q}^{\mathcal{D}}: \mathcal{D} \times \mathcal{D} \rightarrow \widetilde{H}_{\mathbb{C}} \quad \text { with } \quad \widetilde{Q}^{\mathcal{D}}(0,0)=\mathbf{1} .
$$

As for the tube $\mathcal{T}$, the cocycle

$$
J^{\mathcal{D}}: G(\mathcal{D}) \times \mathcal{D} \rightarrow H_{\mathbb{C}}, \quad J^{\mathcal{D}}(g, z):=\mathrm{d} g(z)
$$

lifts to a cocycle

$$
\widetilde{J}^{\mathcal{D}}: \widetilde{G}(\mathcal{D}) \times \mathcal{D} \rightarrow \widetilde{H}_{\mathbb{C}}
$$

As

$$
J^{\mathcal{D}}(k, z)=k \quad \text { for } \quad k \in K(\mathcal{D})=G(\mathcal{D})^{0},
$$

the restriction of $J^{\mathcal{D}}$ provides an inclusion

$$
J^{\mathcal{D}}: K(\mathcal{D})=G(\mathcal{D})^{0} \hookrightarrow H_{\mathbb{C}}
$$

(cf. FK94 Thm. X.5.3, Prop. X.3.1]). Its image is a maximal compact subgroup, so that $H_{\mathbb{C}} \cong K(\mathcal{D})_{\mathbb{C}}$.

\subsection{Unitary representations}

Now we turn to unitary representations of the simply connected groups $\widetilde{G}(\mathcal{D})$ and $\widetilde{G}(\mathcal{T})$ on Hilbert spaces of holomorphic functions on $\mathcal{D}$ and $\mathcal{T}$, respectively. We start with a holomorphic involutive representation $(\rho, \mathcal{K})$ of the complex involutive Lie group $\widetilde{H}_{\mathbb{C}}$ with $g^{*}=\bar{\theta}(g)^{-1}$ on the complex Hilbert space $\mathcal{K}$. This representation does not have to be finite dimensional, but holomorphy implies that the operators of the derived representations are all bounded and that $\rho$ is norm-continuous. Conversely, every norm-continuous $*$-representation of $\widetilde{H}$ defines a holomorphic representation of its universal complexification $\widetilde{H}_{\mathbb{C}}$.

Definition 4.3. A holomorphic $*$-representation $(\rho, \mathcal{K})$ of $\widetilde{H}_{\mathbb{C}}$ is said to be $\mathcal{T}$-positive if the kernel

$$
Q_{\rho}:=\rho \circ \widetilde{Q},: \mathcal{T} \times \mathcal{T} \rightarrow B(\mathcal{K}), \quad Q_{\rho}(z, w)=P_{\rho}\left(\frac{z-\bar{w}}{2 i}\right)
$$

is positive definite.

Then we obtain a representation of $\widetilde{G}(\mathcal{D})$ on the space $\operatorname{Hol}(\mathcal{D}, \mathcal{K})$ by

$$
\left(U_{\rho}^{\mathcal{D}}(g) f\right)(z):=J_{\rho}^{\mathcal{D}}\left(g, g^{-1} . z\right) f\left(g^{-1} . z\right) \quad \text { for } \quad g \in \widetilde{G}(\mathcal{D}), z \in \mathcal{D}, J_{\rho}^{\mathcal{D}}:=\rho \circ \widetilde{J}^{\mathcal{D}},
$$

and a representation of $\widetilde{G}(\mathcal{T})$ on the space $\operatorname{Hol}(\mathcal{T}, \mathcal{K})$ by

$$
\left(U_{\rho}(g) f\right)(z):=J_{\rho}\left(g, g^{-1} . z\right) f\left(g^{-1} . z\right) \quad \text { for } \quad g \in \widetilde{G}(\mathcal{T}), z \in \mathcal{T}, J_{\rho}:=\rho \circ \widetilde{J} .
$$

The space $\operatorname{Hol}(\mathcal{D}, \mathcal{K})$ contains a unitary subrepresentation of $G(\mathcal{D})$ if and only if the kernel $Q_{\rho}^{\mathcal{D}}:=\rho \circ \widetilde{Q}^{\mathcal{D}}$ is positive definite ([Ne99, Prop. XII.2.1]; see also [Ne99, Thm. XII.2.6] for the link to highest weight representations). To see that the Cayley transform provides a natural intertwining operator, we use a holomorphic lift $\widetilde{P}: \mathcal{T} \rightarrow \widetilde{H}_{\mathbb{C}}$ of the quadratic representation $P$ (cf. (42)). As

$$
J(c, z):=J_{c}(z):=\mathrm{d} c(z)=\mathrm{d} p^{-1}(z)=\mathrm{d} p(c(z))^{-1}=\frac{1}{2 i} P(z+i e)=\frac{2}{i} P\left(\frac{z+i e}{2}\right),
$$


up to a central factor $c_{0} \in Z\left(\widetilde{H}_{\mathbb{C}}\right)$,

$$
\widetilde{J}_{c}(z):=c_{0} \widetilde{P}(z+i e)
$$

is a lift of $J_{c}$.

For $g \in \widetilde{G}(\mathcal{D})$ and $\widetilde{g}:=\widetilde{\alpha}_{c}(g) \in \widetilde{G}(\mathcal{T})$, we have $\widetilde{g} \cdot(c(z))=c(g . z)$, and thus

$$
J_{\widetilde{g}}(c(z)) J_{c}(z)=J_{c}(g . z) J_{g}(z) \quad \text { for } \quad z \in \mathcal{D} .
$$

For any holomorphic lift $\widetilde{J}_{c}$ of $J_{c}$, we thus have the relation

$$
\widetilde{J}_{\widetilde{g}}(c(z)) \widetilde{J}_{c}(z) \widetilde{J}_{g}(z)^{-1} \widetilde{J}_{c}(g \cdot z)^{-1} \in \operatorname{ker}\left(q_{H_{\mathbb{C}}}\right) .
$$

For $g=e$, this element is the identity of $\widetilde{H}_{\mathbb{C}}$, and since $\operatorname{ker}\left(q_{H_{\mathbb{C}}}\right)$ is discrete,

$$
\widetilde{J}_{\widetilde{g}}(c(z)) \widetilde{J}_{c}(z) \widetilde{J}_{g}(z)^{-1} \widetilde{J}_{c}(g . z)^{-1}=e \quad \text { for } \quad g \in \widetilde{G}(\mathcal{D}), z \in \mathcal{D} .
$$

This shows that

$$
\widetilde{J}_{\widetilde{g}}(c(z)) \widetilde{J}_{c}(z)=\widetilde{J}_{c}(g . z) \widetilde{J}_{g}(z) \quad \text { for } \quad g \in \widetilde{G}(\mathcal{D}), z \in \mathcal{D} .
$$

From (47) we first derive the relation

$$
\widetilde{Q}^{\mathcal{D}}(z, w)=\widetilde{P}\left(\frac{c(z)+i e}{2}\right)^{-1} \widetilde{Q}(c(z), c(w))\left(\widetilde{P}\left(\frac{c(w)+i e}{2}\right)^{-1}\right)^{*} .
$$

Composing with the $*$-representation $\rho$ and putting $P_{\rho}:=\rho \circ \widetilde{P}$, now leads to

$$
Q_{\rho}^{\mathcal{D}}(z, w)=P_{\rho}\left(\frac{c(z)+i e}{2}\right)^{-1} Q_{\rho}(c(z), c(w)) P_{\rho}\left(\frac{c(w)+i e}{2}\right)^{-*}
$$

This relation implies in particular that the kernel $\mathcal{Q}_{\rho}^{\mathcal{D}}$ on $\mathcal{D} \times \mathcal{D}$ is positive definite if and only if the kernel $\mathcal{Q}_{\rho}$ on $\mathcal{T} \times \mathcal{T}$ is positive definite, i.e., if the representation $\rho$ is $\mathcal{T}$-positive (cf. [Ne99, Thm. I.1.4]). From now one we assume that this is the case. Let $\mathcal{H}_{\rho}^{\mathcal{D}} \subseteq \operatorname{Hol}(\mathcal{D}, \mathcal{K})$ and $\mathcal{H}_{\rho} \subseteq \operatorname{Hol}(\mathcal{T}, \mathcal{K})$ denote the reproducing kernel Hilbert spaces with kernel $Q_{\rho}^{\mathcal{D}}$ and $Q_{\rho}$, respectively.

Lemma 4.4. The map

$$
\Gamma: \operatorname{Hol}(\mathcal{T}, \mathcal{K}) \rightarrow \operatorname{Hol}(\mathcal{D}, \mathcal{K}), \quad \Gamma(f)(z):=P_{\rho}\left(\frac{z+i e}{2}\right)^{-1} f(c(z)),
$$

intertwines the representation of the groups $G(\mathcal{T})$ and $G(\mathcal{D})$ in the sense that

$$
\Gamma \circ U_{\rho}\left(\widetilde{\alpha}_{c}(g)\right)=U_{\rho}^{\mathcal{D}}(g) \circ \Gamma \quad \text { for } \quad g \in \widetilde{G}(\mathcal{D}) .
$$

If the kernel $Q_{\rho}$ is positive definite, then it restricts to a unitary operator $\Gamma: \mathcal{H}_{\rho} \rightarrow \mathcal{H}_{\rho}^{\mathcal{D}}$.

Proof. First we show that the slightly modified map $\Gamma_{0}(f)(z):=\widetilde{J}_{\rho}(c, z)^{-1} f(c(z))$ intertwines the two representation. For $\widetilde{g}:=\widetilde{\alpha}_{c}(g) \in \widetilde{G}(\mathcal{T})$ and $f \in \operatorname{Hol}(\mathcal{D}, \mathcal{K})$ we have

$$
\begin{aligned}
\Gamma_{0}\left(U_{\rho}(\widetilde{g}) f\right)(z) & =\widetilde{J}_{\rho}(c, z)^{-1} \widetilde{J}_{\rho}\left(\widetilde{g}^{-1}, c(z)\right)^{-1} f\left(\widetilde{g}^{-1} \cdot c(z)\right) \\
& =\left(\widetilde{J}_{\rho}\left(\widetilde{g}^{-1}, c(z)\right) \widetilde{J}_{\rho}(c, z)\right)^{-1} f\left(c\left(g^{-1} \cdot z\right)\right) \\
& \stackrel{522}{=}\left(\widetilde{J}_{\rho}\left(c, g^{-1} \cdot z\right) \widetilde{J}_{\rho}\left(g^{-1}, z\right)\right)^{-1} f\left(c\left(g^{-1} \cdot z\right)\right) \\
& =\widetilde{J}_{\rho}\left(g^{-1}, z\right)^{-1} \widetilde{J}_{\rho}\left(c, g^{-1} \cdot z\right)^{-1} f\left(c\left(g^{-1} \cdot z\right)\right) \\
& =\left(U_{\rho}(g) \Gamma_{0}(f)\right)(z) .
\end{aligned}
$$

\footnotetext{
${ }^{4}$ There is a certain freedom in normalizing the kernel on the tube domain. We have chosen a normalization for which $Q_{\rho}(i e, i e)=\operatorname{id}_{\mathcal{K}}$, and this is why we use the $P_{\rho}$-factors to transform the kernels and not the factors $J_{\rho}\left(c, c^{-1}(z)\right)$ obtained from the differential of the Cayley transform $c: \mathcal{D} \rightarrow \mathcal{T}$. The source of the trouble is the fact that $d c(0)=d p(i e)^{-1}=2 i 1$ is not unitary.
} 
Here we have used the relation

$$
\widetilde{J}_{\rho}\left(\widetilde{g}^{-1}, c(z)\right) \widetilde{J}_{\rho}(c, z)=\widetilde{J}_{\rho}\left(c, g^{-1} \cdot z\right) \widetilde{J}_{\rho}\left(g^{-1}, z\right)
$$

which follows from (52). Next we observe that, by (51), there exists a central element $z \in \widetilde{H}_{\mathbb{C}}$ such that $\Gamma(f)=\rho(z) \Gamma_{0}(f)$. As composition with $\rho(z)$ commutes with the representation of $\widetilde{G}(\mathcal{D})$, this implies the intertwining property of $\Gamma$.

To see that $\Gamma$ restrict to a unitary operator $\mathcal{H}_{\rho} \rightarrow \mathcal{H}_{\rho}^{\mathcal{D}}$, we show that it maps $\mathcal{H}_{\rho}$ isometrically onto the subspace with reproducing kernel $Q_{\rho}^{\mathcal{D}}$. Writing, for simplicity, $\Gamma(f)(z)=\beta(z) f(c(z))$ with $\beta(z):=P_{\rho}\left(\frac{z+i e}{2}\right)^{-1}$, the injective map $\Gamma$ maps $\mathcal{H}_{\rho}$ unitarily onto a reproducing kernel Hilbert space $\operatorname{im}(\Gamma) \subseteq \operatorname{Hol}(\mathcal{D}, \mathcal{K})$ on which the point evaluations are given by

$$
\mathrm{ev}_{z}=\beta(z) \circ \mathrm{ev}_{c(z)} \circ \Gamma^{-1}=\beta(z) Q_{\rho, c(z)} \Gamma^{*} .
$$

Therefore the reproducing kernel of $\operatorname{im}(\Gamma)$ is given by

$$
\begin{aligned}
\operatorname{ev}_{z} \operatorname{ev}_{w}^{*} & =\beta(z) Q_{\rho, c(z)} \Gamma^{*} \Gamma Q_{\rho, c(w)}^{*} \beta(w)^{*}=\beta(z) Q_{\rho, c(z)} Q_{\rho, c(w)}^{*} \beta(w)^{*} \\
& =\beta(z) Q_{\rho}(c(z), c(w)) \beta(w)^{*} \stackrel{544}{=} Q_{\rho}^{\mathcal{D}}(z, w) .
\end{aligned}
$$

This implies that $\Gamma\left(\mathcal{H}_{\rho}\right)=\mathcal{H}_{\rho}^{\mathcal{D}}$ and that $\left.\Gamma\right|_{\mathcal{H}_{\rho}}$ is unitary $($ Ne99, Lemma I.1.5]).

\subsection{Antiunitary extensions}

In this subsection we discuss antiunitary extensions of the unitary representations of $\widetilde{G}(\mathcal{D})$ and $\widetilde{G}(\mathcal{T})$ on $\mathcal{H}_{\rho}^{\mathcal{D}}$ and $\mathcal{H}_{\rho}$ respectively. We refer to NÓ17 for more background on antiunitary representations. We are mainly interested in antiunitary extensions to the simply connected group $\widetilde{G}_{\tau} \cong \widetilde{G}(\mathcal{T})_{\tau}$. The involution $\tau$ acts on $\mathcal{T}$ and $\mathcal{D}$ by

$$
\tau_{\mathcal{T}}(z)=-\bar{z} \quad \text { and } \quad \tau_{\mathcal{D}}(z)=\bar{z} .
$$

From

$$
\tau_{\mathcal{T}}(c(z))=i(e+\bar{z})(e-\bar{z})^{-1}=c(\bar{z})=c\left(\tau_{\mathcal{D}}(z)\right)
$$

we derive that the Cayley transform intertwines $\tau_{\mathcal{T}}$ with $\tau_{\mathcal{D}}$. We define

$$
\tau_{G}(g):=\tau_{\mathcal{D}} \circ g \circ \tau_{\mathcal{D}} \quad \text { for } \quad g \in G(\mathcal{D}), \quad \text { and } \quad \tau_{G}(g):=\tau_{\mathcal{T}} \circ g \circ \tau_{\mathcal{T}} \quad \text { for } \quad g \in G(\mathcal{T}) .
$$

Then (55) implies that

$$
c \tau_{G}(g) c^{-1}=\tau_{G}\left(c g c^{-1}\right) \quad \text { for } \quad g \in \mathcal{G}(\mathcal{D}) .
$$

Definition 4.5. Let $\widetilde{G}(\mathcal{D})$ denote the universal covering of the group $G(\mathcal{D})=\operatorname{Aut}(\mathcal{D})_{0}$ and $\tau_{G}$ denote the involution of $\widetilde{G}(\mathcal{D})$ induced by conjugating with $\tau_{\mathcal{D}}$. An antiunitary representation $(U, \mathcal{H})$ of

$$
\widetilde{G}(\mathcal{D})_{\tau}:=\widetilde{G}(\mathcal{D}) \rtimes\left\{\mathbf{1}, \tau_{G}\right\}
$$

is called a positive energy representation if

$$
-i \mathrm{~d} U(\mathbf{d}) \geq 0 \quad \text { holds for } \quad \mathbf{d}:=-i h .
$$

Positive energy representations $(U, \mathcal{H})$ of $\widetilde{G}(\mathcal{D})_{\tau}$ are direct sums of representations which are holomorphically induced from unitary representations of $\widetilde{K}(\mathcal{D})$ (see Ne13] for details and definitions), i.e., realized in reproducing kernel spaces $\mathcal{H}_{\rho}^{\mathcal{D}} \subseteq \operatorname{Hol}(\mathcal{D}, \mathcal{K})$ for norm-continuous unitary representations $(\rho, \mathcal{K})$ of $\widetilde{K}(\mathcal{D})$. 
For any such representation, the unitary one-parameter group $U_{t}:=U(\exp t \mathbf{d})$ acts on $f \in \mathcal{H}_{\rho}^{\mathcal{D}}$ by

$$
\left(U_{t} f\right)(z)=\rho(\exp t \mathbf{d}) f(\exp (-t \mathbf{d}) . z)=\rho(\exp t \mathbf{d}) f\left(e^{i t} z\right)
$$

so that

$$
-i \partial U(\mathbf{d}) f=-i \partial \rho(\mathbf{d}) f+\mathrm{d} f(z) z
$$

where $(E f)(z):=\mathrm{d} f(z) z$ is the Euler operator on holomorphic functions. Therefore

$$
\operatorname{Spec}(-i \partial U(\mathbf{d})) \subseteq \operatorname{Spec}(-i \partial \rho(\mathbf{d}))+\mathbb{N}_{0} .
$$

As $\rho$ is norm-continuous, the operator $\partial \rho(\mathbf{d})$ is bounded, and thus $-i \partial U(\mathbf{d})$ is bounded from below. If, in addition, $(\rho, \mathcal{K})$ is irreducible, then $-i \mathrm{~d} \rho(\mathbf{d})=\zeta \mathbf{1}$ for some $\zeta \in \mathbb{R}$ and

$$
-i \partial U(\mathbf{d}) f=\zeta f+\mathrm{d} f(z) z
$$

shows that $\mathcal{K}=\operatorname{ker}(i \partial U(\mathbf{d})+\zeta \mathbf{1})$ is the minimal eigenspace of $-i \partial U(\mathbf{d})$. Here we use that, if $f: \mathcal{D} \rightarrow \mathcal{K}$ is holomorphic and $f=\sum_{n=0}^{\infty} f_{n}$ is its Taylor expansion, then

$$
\mathrm{d} f(z) z=\sum_{n=0}^{\infty} n f_{n}(z) .
$$

We recall that the positive definiteness of the kernel $Q_{\rho}^{\mathcal{D}}$ implies that, for the corresponding unitary representation $U_{\rho}^{\mathcal{D}}$, the operator $-i \partial U_{\rho}^{\mathcal{D}}(\mathbf{d})$ is bounded from below. As $\mathbf{d}$ belongs to a minimal invariant cone in $\mathfrak{g}$, it even follows that $-i \partial U_{\rho}^{\mathcal{D}}(\mathbf{d}) \geq 0$ and hence that $-i \partial \rho(\mathbf{d}) \geq 0$ (see [Ne99, Thms. X.4.1, XIV.1.3]). Actually the positive cone $C_{\rho} \subseteq \mathfrak{k}(\mathcal{D})$ contains the intersection $C_{U_{\rho}^{\mathcal{D}}} \cap \mathfrak{k}(\mathcal{D})$, which has interior points.

Proposition 4.6. Suppose that the representation $(\rho, \mathcal{K})$ of $\widetilde{H}_{\mathbb{C}}$ is irreducible. For the representations $\left(U_{\rho}^{\mathcal{D}}, \mathcal{H}_{\rho}^{\mathcal{D}}\right)$ of $\widetilde{G}(\mathcal{D})$ and $\left(U_{\rho}, \mathcal{H}_{\rho}\right)$ of $\widetilde{G}(\mathcal{T})$, the following are equivalent:

(a) $\left(U_{\rho}^{\mathcal{D}}, \mathcal{H}_{\rho}^{\mathcal{D}}\right)$ extends to an antiunitary representation of $\widetilde{G}(\mathcal{D})_{\tau}$.

(b) $(\rho, \mathcal{K})$ extends to an antilinear representation of $\widetilde{H}_{\mathbb{C}, \tau}$, i.e., there exists a conjugation $J_{\mathcal{K}}$ on $\mathcal{K}$ satisfying $J_{\mathcal{K}} \rho(g) J_{\mathcal{K}}=\rho\left(\tau_{\widetilde{H}_{\mathbb{C}}}(g)\right)$ for $g \in \widetilde{H}_{\mathbb{C}}$.

(c) $\left(U_{\rho}, \mathcal{H}_{\rho}\right)$ extends to an antiunitary representation of $\widetilde{G}(\mathcal{T})_{\tau}$.

Proof. $(\mathrm{a}) \Rightarrow(\mathrm{b})$ : Suppose that $\left(U_{\rho}^{\mathcal{D}}, \mathcal{H}_{\rho}^{\mathcal{D}}\right)$ extends to an antiunitary representation of $\widetilde{G}(\mathcal{D})_{\tau}$ by $J:=U_{\rho}^{\mathcal{D}}\left(\tau_{G}\right)$. Then

$$
J\left(-i \partial U_{\rho}^{\mathcal{D}}(\mathbf{d})\right) J=i \partial U_{\rho}^{\mathcal{D}}(\tau(\mathbf{d}))=i \partial U_{\rho}^{\mathcal{D}}(-\mathbf{d})=-i \partial U_{\rho}^{\mathcal{D}}(\mathbf{d})
$$

implies that $J$ commutes with $i \mathrm{~d} U_{\rho}^{\mathcal{D}}(\mathbf{d})$, hence preserves its eigenspaces and in particular the minimal eigenspace $\mathcal{K}$ of constant functions. Therefore the restriction $J_{\mathcal{K}}:=\left.J\right|_{\mathcal{K}}$ defines an antiunitary extension of the unitary representation $(\rho, \mathcal{K})$ of $\widetilde{K}(\mathcal{D})$ to the group $\widetilde{K}(\mathcal{D})_{\tau}=$ $\widetilde{K}(\mathcal{D}) \rtimes\{\mathbf{1}, \tau\}$. As $\widetilde{K}(\mathcal{D})$ and $\widetilde{H}_{\mathbb{C}}$ are simply connected, and the homomorphism $\widetilde{J}: \widetilde{K}(\mathcal{D}) \rightarrow$ $\widetilde{H}_{\mathbb{C}}$ induces on the Lie algebra level an inclusion $\mathfrak{k}(\mathcal{D}) \rightarrow \mathfrak{h}_{\mathbb{C}}$ of a real form, $\widetilde{J}$ defines the universal complexification of $\widetilde{K}(\mathcal{D})$. Hence the finite dimensional representation $\rho$ extends to a holomorphic representation of $\widetilde{H}_{\mathbb{C}}$, so that we obtain a representation of $\widetilde{H}_{\mathbb{C}, \tau}$.

(b) $\Rightarrow(\mathrm{c})$ : Let $J_{\mathcal{K}}$ be a conjugation on $\mathcal{K}$ extending the representation $\rho$ to $\widetilde{H}_{\mathbb{C}, \tau}$. We define

$$
(J f)(z):=J_{\mathcal{K}} f(\tau(z))=J_{\mathcal{K}} f(-\bar{z}) .
$$

It is clear that $J$ is an antilinear involution on $\operatorname{Hol}(\mathcal{T}, \mathcal{K})$. To see that it is antiunitary on $\mathcal{H}_{\rho}$, we have to verify that

$$
Q_{\rho}(-\bar{z},-\bar{w})=J_{\mathcal{K}} Q_{\rho}(w, z) J_{\mathcal{K}} \quad \text { for } \quad z, w \in \mathcal{D} .
$$


The left hand side is given by

$$
Q_{\rho}(-\bar{z},-\bar{w})=\rho(\widetilde{Q}(-\bar{z},-\bar{w}))
$$

and the right hand side by

$$
J_{\mathcal{K}} Q_{\rho}(w, z) J_{\mathcal{K}}=\rho(\tau \widetilde{Q}(w, z) \tau)=\rho\left(\tau_{\widetilde{H}_{\mathbb{C}}}(\widetilde{Q}(w, z))\right) .
$$

By the uniqueness of lifts, it therefore suffices to verify that

$$
\tau_{H_{\mathbb{C}}}(Q(w, z))=Q(\tau(z), \tau(w)) \quad \text { for } \quad z, w \in \mathcal{D} .
$$

We start with the observation that

$$
P(-\bar{z})=P(\bar{z})=\overline{P(z)}=\tau_{H_{\mathbb{C}}}(P(z)) \quad \text { for } \quad z \in E_{\mathbb{C}}^{\times} .
$$

We further have

$$
Q(\tau(z), \tau(w))=Q(-\bar{z},-\bar{w})=P\left(\frac{-\bar{z}+w}{2 i}\right)=Q(w, z)
$$

which leads to

$$
\begin{aligned}
Q(\tau(z), \tau(w)) & =Q(w, z)=P\left(\frac{w-\bar{z}}{2 i}\right)=\tau_{H_{\mathbb{C}}}\left(P\left(\frac{-\bar{w}+z}{-2 i}\right)\right) \\
& =\tau_{H_{\mathbb{C}}}\left(P\left(\frac{z-\bar{w}}{2 i}\right)\right)=\tau_{H_{\mathbb{C}}}(Q(z, w)) .
\end{aligned}
$$

This proves (58). Lifting (60) to $\widetilde{H}_{\mathbb{C}}$ and composing with the extension of $\rho$ to $\widetilde{H}_{\mathbb{C}, \tau}$ by $\rho(\tau):=$ $J_{\mathcal{K}}$, we get

$$
Q_{\rho}(\tau(z), \tau(w))=J_{\mathcal{K}} Q_{\rho}(z, w) J_{\mathcal{K}} .
$$

This implies that $(J f)(z)=J_{\mathcal{K}} f(-\bar{z})$ defines an antiunitary involution on $\mathcal{H}_{\rho} \subseteq \operatorname{Hol}(\mathcal{T}, \mathcal{K})$.

Next we show that $U_{\rho}(\tau):=J$ defines an antiunitary extension of $U_{\rho}$ to $\widetilde{G}(\mathcal{T})_{\tau}$. As $\tau(g)=$ $\tau g \tau$ holds in $\operatorname{Diff}(\mathcal{T})$ for any $g \in G(\mathcal{T})$, we obtain for $g \in \widetilde{G}(\mathcal{T})$ the relation

$$
\left(J U_{\rho}(g) f\right)(z)=J_{\mathcal{K}} J_{\rho}\left(g, g^{-1} \cdot \tau(z)\right) f\left(g^{-1} \cdot \tau(z)\right) \stackrel{!}{=} J_{\rho}\left(\tau(g), \tau(g)^{-1} \cdot z\right) J_{\mathcal{K}} f\left(\tau\left(\tau(g)^{-1} \cdot z\right)\right)
$$

follows from

$$
\begin{aligned}
\tau_{H_{\mathbb{C}}}\left(J\left(g, g^{-1} \cdot \tau(z)\right)\right) & =\tau_{H_{\mathbb{C}}}\left(J\left(g^{-1}, \tau(z)\right)\right)^{-1}=\tau_{H_{\mathbb{C}}}\left(\mathrm{d}\left(g^{-1}\right)(-\bar{z})\right)^{-1} \\
& =\mathrm{d}\left(\tau(g)^{-1}\right)(z)^{-1}=J\left(\tau(g), \tau(g)^{-1} \cdot z\right) .
\end{aligned}
$$

(c) $\Rightarrow$ (a): Since we have a unitary intertwining operator $\Gamma: \mathcal{H}_{\rho} \rightarrow \mathcal{H}_{\rho}^{\mathcal{D}}$ (Lemma 4.4), it suffices to show that $\Gamma$ is also compatible with the involutions $\tau_{G}$ on the groups $\widetilde{G}(\mathcal{D})$ and $\widetilde{G}(\mathcal{T})$ in the sense that

$$
\Gamma \circ U_{\rho}\left(\tau_{G}(g)\right)=U_{\rho}^{\mathcal{D}}\left(\tau_{G}(g)\right) \circ \Gamma \quad \text { for } \quad g \in \widetilde{G}(\mathcal{T})
$$

As

$$
\left.\Gamma \circ U_{\rho}\left(\widetilde{\alpha}_{c}(g)\right)\right)=U_{\rho}^{\mathcal{D}}(g) \circ \Gamma
$$

by Lemma 4.4 the assertion follows from (56), which, in view of the uniqueness of lifts, implies that $\widetilde{\alpha}_{c}\left(\tau_{G}(g)\right)=\tau_{G}\left(\widetilde{\alpha}_{c}(g)\right)$ for $g \in \widetilde{G}(\mathcal{D})$.

Lemma 4.7. The homomorphism

$$
\widetilde{J}: \widetilde{G}^{\tau} \rightarrow \widetilde{H}_{\mathbb{C}}
$$

is injective and defines the universal complexification of $\widetilde{G}^{\tau_{G}}$. In particular, it defines an isomorphism $\widetilde{G}^{\tau_{G}} \rightarrow H^{\sharp}=\left\langle\exp _{\widetilde{H}_{\mathbb{C}}} \mathfrak{h}\right\rangle$. 
Proof. Since the image of $\widetilde{J}$ is the connected Lie subgroup with Lie algebra $\mathfrak{h}$, it suffices to show that it is injective.

(a) Recall that $\widetilde{J}^{\mathcal{D}}: \widetilde{K}(\mathcal{D}) \rightarrow \widetilde{H}_{\mathbb{C}}$ is the universal complexification of the simply connected group $\widetilde{K}(\mathcal{D})$. We know from the Gelfand-Raikov Theorem ( Ne99, Thm. XI.5.2]) that the contractive holomorphic representations of $S_{C}$ separate the points of $S_{C}$. This implies that the $\mathcal{T}$-positive representations of $\widetilde{H}_{\mathbb{C}}$ separate the points of $\widetilde{H}_{\mathbb{C}}$ because composition with $\widetilde{J}^{\mathcal{D}}$ separates the points of $\widetilde{K}(\mathcal{D})$.

(b) Now let $g \in \widetilde{G}^{\tau_{G}}$ with $\widetilde{J}(g)=e$. As the action of $\widetilde{G}^{\tau_{G}}$ on $E_{\mathbb{C}}$ factors through the action of $\widetilde{H}_{\mathbb{C}}$, defined by the covering map into $H_{\mathbb{C}}, g$ acts trivially on $\mathcal{T}$. As $\widetilde{J}(g)=e$ also acts trivially on $\mathcal{K}$ for every $\mathcal{T}$-positive representation $(\rho, \mathcal{K})$, it follows that $U_{\rho}(g)=\mathbf{1}$. From (a) we now derive that $g=e$.

Remark 4.8. As any $\mathcal{T}$-positive norm-continuous representation $(\rho, \mathcal{K})$ of $\widetilde{H}_{\tau}$ defines a holomorphic representation of its universal complexification, we have $\operatorname{ker} \rho \supseteq \operatorname{ker}\left(\eta_{\widetilde{H}}\right)$, i.e., $\rho$ factors through a representation of the subgroup $H^{\sharp} \subseteq \widetilde{H}_{\mathbb{C}}$. In the preceding lemma we have seen that this subgroup can be identified with $\widetilde{G}^{\tau_{G}}$ via the isomorphism $\widetilde{J}$.

Example 4.9. For the Jordan algebra $E=\operatorname{Sym}_{n}(\mathbb{R}), \mathfrak{g}=\mathfrak{s p}_{2 n}(\mathbb{R})$ and $\mathfrak{h}=\mathfrak{g l}_{n}(\mathbb{R})$, we have

$$
\widetilde{H}_{\mathbb{C}} \cong \widetilde{\mathrm{GL}}_{n}(\mathbb{C}) \cong \mathbb{C} \times \mathrm{SL}_{n}(\mathbb{C}) .
$$

The real subgroup corresponding to $\mathfrak{h}$ is the non-simply connected group $H^{\sharp} \cong \mathbb{R} \times \mathrm{SL}_{n}(\mathbb{R})$. The natural map $H^{\sharp} \rightarrow \mathrm{Sp}_{2 n}(\mathbb{R})$ lifts to a homomorphism $H^{\sharp} \rightarrow{\widetilde{\mathrm{Sp}_{2 n}}}_{2 n}(\mathbb{R})$ because the inclusion $\mathrm{SO}_{n}(\mathbb{R}) \hookrightarrow \mathrm{U}_{n}(\mathbb{C}) \subseteq \mathrm{Sp}_{2 n}(\mathbb{R})$ induces the trivial homomorphism $\pi_{1}\left(\mathrm{SO}_{n}(\mathbb{R})\right) \rightarrow \pi_{1}\left(\mathrm{Sp}_{2 n}(\mathbb{R})\right)$.

Remark 4.10. (a) In the proof of Proposition 4.6 the implications (b) $\Rightarrow$ (c) $\Rightarrow$ (a) hold for any $\mathcal{T}$-positive holomorphic $*$-representation $(\rho, \mathcal{K})$ of $\widetilde{H}_{\mathbb{C}}$. Only the implication $(\mathrm{a}) \Rightarrow(\mathrm{b})$ requires additional assumptions; such as irreducibility.

(b) If the conditions of Proposition 4.6 are satisfied, then the unitary intertwining operator $\Gamma: \mathcal{H}_{\rho} \rightarrow \mathcal{H}_{\rho}^{\mathcal{D}}$ intertwines the conjugation $J$ on $\mathcal{H}_{\rho} \subseteq \operatorname{Hol}(\mathcal{T}, \mathcal{K})$ defined by $(J f)(z):=J_{\mathcal{K}} f(-\bar{z})$ with the conjugation $J^{\mathcal{D}}$ on $\mathcal{H}_{\rho}^{\mathcal{D}}$ defined by $\left(J^{\mathcal{D}} f\right)(z):=J_{\mathcal{K}} f(-\bar{z})$. In fact, we have

$$
\left(J^{\mathcal{D}} \Gamma(f)\right)(z)=J_{\mathcal{K}} P_{\rho}\left(\frac{-\bar{z}+i e}{2}\right) f(c(-\bar{z}))=P_{\rho}\left(\frac{z+i e}{2}\right) J_{\mathcal{K}} f(-\overline{c(z)})=\Gamma(J f)(z) .
$$

(c) For any unitary representation $(U, \mathcal{H})$ of $\widetilde{G}$, the direct sum $U \oplus\left(U^{*} \circ \tau_{G}\right)$ on $\mathcal{H} \oplus \mathcal{H}^{*}$ extends to an antiunitary representation of $\widetilde{G}_{\tau}$, so that the situations of Proposition 4.6 can always be achieved by this doubling process (cf. [NÓ17, Lemma 2.10]). The same argument applies to a holomorphic $*$-representation $(\rho, \mathcal{K})$ of the symmetric Lie group $\left(\widetilde{H}_{\mathbb{C}}, \bar{\theta}\right)$, for which the representation $\rho \oplus\left(\rho^{*} \circ \bar{\theta}\right)$ on $\mathcal{K} \oplus \mathcal{K}^{*}$ always extends to $\widetilde{H}_{\mathbb{C}, \tau}$.

(d) Uniqueness of extensions: From [NÓ17, Thm. 2.11] we know that any two extensions of a unitary representation $(U, \mathcal{H})$ of $\widetilde{G}$ to an antiunitary representation of $\widetilde{G}_{\tau}$ are unitarily equivalent, regardless of whether the representation $U$ is irreducible or not.

(e) Irreducible holomorphic representations $(\rho, \mathcal{K})$ of $\left(\widetilde{H}_{\mathbb{C}}, \bar{\theta}\right)$ are finite dimensional and every finite dimensional irreducible complex representation $(\beta, \mathcal{K})$ of the complex Lie algebra $\mathfrak{h}_{\mathbb{C}}$ on a complex vector space $\mathcal{K}$ integrates to a holomorphic representation $(\rho, \mathcal{K})$ of $\widetilde{H}_{\mathbb{C}}$. Moreover, $\mathcal{K}$ carries a Hilbert space structure for which $\rho$ is a $*$-representation if and only if, for each $x \in \mathfrak{z}(\mathfrak{h})$, the operator $\beta(x)$ is diagonalizable with real eigenvalues. Here we use that $\theta(x)=x$ for $x \in \mathfrak{z}(\mathfrak{h})$. Then the subgroup $\widetilde{H}_{\mathbb{C}}^{\bar{\theta}}$ is represented by a compact group and the scalar product can be obtained by averaging any given scalar product on $\mathcal{K}$.

(f) A representation of $\widetilde{H}_{\mathbb{C}}$ extends to $\widetilde{H}_{\mathbb{C}, \tau}$ if and only if there exists a conjugation $J_{\mathcal{K}}$ on $\mathcal{K}$ commuting with $\beta(\mathfrak{h})$. This is equivalent to the representation $(\beta, \mathcal{K})$ of the Lie algebra $\mathfrak{h}$ being real in the sense that $\mathcal{K}_{\mathbb{R}}:=\operatorname{Fix}\left(J_{\mathcal{K}}\right)$ is a real $\mathfrak{h}$-invariant subspace and the representation on $\mathcal{K}$ arising by complex linear extension. Conversely, every real $*$-representation $\left(\rho, \mathcal{K}_{\mathbb{R}}\right)$ of 
$\mathfrak{h}$, resp., the group $H^{\sharp} \subseteq \widetilde{H}_{\mathbb{C}}$, extends to a complex $*$-representation $\left(\rho,\left(\mathcal{K}_{\mathbb{R}}\right)_{\mathbb{C}}\right)$ of $H_{\tau}^{\sharp}$ by $\rho(\tau)(x+i y):=x-i y$ and this representation extends to a holomorphic representation of the universal complexification $\widetilde{H}_{\mathbb{C}}$. Therefore the irreducible extendable representations of $\widetilde{H}_{\mathbb{C}, \tau}$ correspond to the irreducible real $*$-representations of $\mathfrak{h}$.

(g) The classification of real irreducible representations can easily be reduced to the classification of the complex ones by the following observations ( On20, Ch. 8], Iw59]). If $(\beta, V)$ is a real simple $\mathfrak{h}$-module, then we say it is of real, complex of quaternionic type if $\operatorname{End}_{\mathfrak{h}}(V) \cong \mathbb{R}, \mathbb{C}$ or $\mathbb{H}$. If $V$ is of real type, then $V_{\mathbb{C}} \cong \overline{V_{\mathbb{C}}}$ is simple. If $V$ is of complex type, then $V$ itself is complex simple with $\bar{V} \approx V$ (there is no antilinear automorphism), and if $V$ is of quaternionic type, then $V \cong \bar{V}$ is simple complex, and there exists an antilinear endomorphism $J$ with $J^{2}=-\mathbf{1}$. We thus obtain for each simple real $\mathfrak{h}$-module a simple complex one, and the three types are reflected in the (non-)existence of antilinear endomorphisms $J$ with $J^{2}= \pm \mathbf{1}$.

Thus every simple complex module $V$ with $\bar{V} \not V$ defines by restriction of scalars a real simple module $V^{\mathbb{R}}$ of complex type, and $\bar{V}^{\mathbb{R}} \cong V^{\mathbb{R}}$. If $\bar{V} \cong V$, then $V$ there exists an antilinear endomorphism $J$ with $J^{2}= \pm \mathbf{1}$. If $J^{2}=\mathbf{1}$, then $V^{J}=\operatorname{Fix}(J)$ is a real simple module of real type, and if $J^{2}=-\mathbf{1}$, then $V^{\mathbb{R}}$ is a real simple module of quaternionic type.

The three types of complex simple modules can be identified in terms of their highest weights. Let $\mathfrak{b} \subseteq \mathfrak{h}^{\theta}$ be maximal abelian and $\mathfrak{a} \subseteq \mathfrak{h}^{-\theta} \cap \mathfrak{z}_{\mathfrak{h}}(\mathfrak{b})$ be maximal abelian, so that $\mathfrak{c}:=\mathfrak{a} \oplus \mathfrak{b}$ is a Cartan subalgebra of $\mathfrak{h}$ which is "maximally compact". In particular $\mathfrak{b}$ contains regular elements of $\mathfrak{h}$. We parametrize irreducible complex representations $\left(\beta_{\lambda}, V_{\lambda}\right)$ of $\mathfrak{h}$ by their highest weights $\lambda: \mathfrak{c} \rightarrow \mathbb{C}$ which are real on $\mathfrak{a}$, purely imaginary on $\mathfrak{b}$ and dominant with respect to a Weyl chamber intersecting $i \mathfrak{b}$, so that the Weyl chamber can be chosen invariant under $\theta$. As the weights of the complex conjugate representation are the complex conjugates of the weights and $-\lambda \circ \theta=\bar{\lambda}$ on $\mathfrak{c}$, the highest weight of the complex conjugate representation is $\overline{V_{\lambda}}$ is $-w \cdot(\lambda \circ \theta)=w \cdot \bar{\lambda}$, where $w \in \mathcal{W}$ is the longest element in the Weyl group. Therefore $\overline{V_{\lambda}} \cong V_{\lambda}$ is equivalent to $\lambda=w \cdot \bar{\lambda}$. If this is the case, then there exists an antilinear endomorphism $J$ with $J^{2}=(-1)^{\varepsilon_{\lambda}} \mathbf{1}$. The number $\varepsilon_{\lambda}$ can be determined from $\lambda$ and the root system; see On20. Thm. 8.3] and the subsequent discussion for details.

\section{$4.4 \quad L^{2}$-realization}

The representations $\left(\rho, \widetilde{H}_{\mathbb{C}}\right)$ that are of interest for us are the $\mathcal{T}$-positive ones. This condition is equivalent to the positive definiteness of the function $P_{\rho}: \mathcal{T} \rightarrow B(\mathcal{K})$ on the $*$-semigroup $\mathcal{T}$ with involution $z^{*}:=-\bar{z}$, which in turn is equivalent to the existence of a unique $\operatorname{Herm}^{+}(\mathcal{K})$-valued measure $\mu$ on $E_{+}^{\star}$ with

$$
P_{\rho}=\mathcal{L}(\mu), \quad \mathcal{L}(\mu)(z)=\int_{E^{*}} e^{-\lambda(z)} d \mu(\lambda)
$$

\section{(cf. HN01, Thm. V.12])}

Lemma 4.11. The measure $\mu$ has the following transformation properties:

(a) $J_{\mathcal{K}} \mu J_{\mathcal{K}}=\mu$, i.e., $\mu$ takes values in the subspace $\mathrm{Sym}^{+}\left(\mathcal{K}^{J_{\mathcal{K}}}\right)$ of positive symmetric operators on real Hilbert space $\mathcal{K}^{J_{\mathcal{K}}}$.

(b) $\left(g^{-1}\right)_{*} \mu=\rho(g) \mu \rho(g)^{*}$ for $g \in \widetilde{H}$; i.e., for every compactly supported continuous function we have

$$
\int_{E^{*}} \varphi\left(g^{-1} \cdot \lambda\right) d \mu(\lambda)=\int_{E^{*}} \varphi(\lambda) \rho(g) d \mu(\lambda) \rho(g)^{*} .
$$

Proof. (a) The relation $\tau_{H_{\mathbb{C}}}(P(z))=\overline{P(z)}=P(\bar{z})=P(-\bar{z})$ from (59) implies that

$$
\mathcal{L}(\mu)(\bar{z})=P_{\rho}(\bar{z})=J_{\mathcal{K}} P_{\rho}(z) J_{\mathcal{K}}=J_{\mathcal{K}} \mathcal{L}(\mu)(z) J_{\mathcal{K}}=\mathcal{L}\left(J_{\mathcal{K}} \mu J_{\mathcal{K}}\right)(\bar{z}) \quad \text { for } \quad z \in E_{+}+i E=-i \mathcal{T} .
$$


As any tempered measure on $E_{+}^{\star}$ is uniquely determined by its Laplace transform, the assertion follows.

(b) Starting from the relation

$$
P_{\rho}(g . z)=\rho(g) P_{\rho}(z) \rho(g)^{*} \quad \text { for } \quad g \in \widetilde{H}, z \in \mathcal{T},
$$

we obtain

$$
\mathcal{L}\left(\left(g^{-1}\right)_{*} \mu\right)(z)=\mathcal{L}(\mu)(g . z)=\rho(g) \mathcal{L}(\mu)(z) \rho(g)^{*}=\mathcal{L}\left(\rho(g) \mu \rho(g)^{*}\right)(z) .
$$

As in (a), this implies (b).

With a slight modification of the normalizations in NÓO20 §3.2] (a factor 2 in the exponent), it follows that

$$
\Phi: L^{2}\left(E^{*}, \mu, \mathcal{K}\right) \rightarrow \operatorname{Hol}(\mathcal{T}, \mathcal{K}), \quad \Phi(f)(z)=\int_{E^{*}} e^{\frac{i \lambda(z)}{2}} d \mu(\lambda) f(\lambda)
$$

maps $L^{2}\left(E^{*}, \mu, \mathcal{K}\right)$ injectively onto the reproducing kernel Hilbert space $\mathcal{H}_{Q}$ with $B(\mathcal{K})$-valued kernel

Note that

$$
Q_{\rho}(z, w)=\mathcal{L}(\mu)\left(\frac{z-\bar{w}}{2 i}\right)=\int_{E^{*}} e^{i \lambda(z-\bar{w}) / 2} d \mu(\lambda) .
$$

$$
\langle\xi, \Phi(f)(z)\rangle=\left\langle e_{-i \bar{z} / 2} \xi, \mu \cdot f\right\rangle \quad \text { for } \quad v \in \mathcal{K}, z \in \mathcal{T} .
$$

The map $\Phi$ is a unitary intertwining operator for the antiunitary representation of the group $E \rtimes \widetilde{H}_{\tau}$ on $L^{2}\left(E^{*}, \mu, \mathcal{K}\right)$, defined by

$$
(U(x, g) f)(\lambda):=e^{-\frac{i \lambda(x)}{2}} \rho^{\theta}(g) f\left(g^{-1} \cdot \lambda\right), \quad U(0, \tau) f:=J_{\mathcal{K}} \circ f,
$$

where $\rho^{\theta}:=\rho \circ \theta$ is the $\theta$-twist of $\rho$. Here we use that the representation of $E \rtimes \widetilde{H}_{\tau}$ on $\mathcal{H}_{\rho} \subseteq \operatorname{Hol}(\mathcal{T}, \mathcal{K})$ has the form

$$
\left(U_{\rho}(x, g) f\right)(z):=\rho(g) f\left(g^{-1} \cdot(z-x)\right) \quad \text { for } \quad g \in \widetilde{H}, x \in E, z \in \mathcal{T},
$$

and

$$
\left(U_{\rho}(\tau) f\right)(z):=J_{\mathcal{K}} f(-\bar{z}) .
$$

Example 4.12. The case where $\mathcal{K}=\mathbb{C}$ is one-dimensional is particularly interesting and easily described. Then the representation $\rho: \widetilde{H} \rightarrow \mathrm{GL}_{1}(\mathbb{R}) \cong \mathbb{R}^{\times}$is a real character. We now take a closer look at these representations for the case where $E$ is simple.

Let $E$ be a simple euclidean Jordan algebra or rank $r$ whose Pierce subspaces are of dimension $d$. For

$$
s \in\left\{0, \cdots,(r-1) \frac{d}{2}\right\} \cup\left((r-1) \frac{d}{2}, \infty\right),
$$

we consider the corresponding Riesz measure $\mu_{s}$ on $E_{+}^{\star}$ whose Fourier (Laplace) transform satisfies

$$
\mathcal{L}\left(\mu_{s}\right)(z)=\widetilde{\mu}_{s}(i z)=\Delta_{E}(z)^{-s} \quad \text { for } \quad z \in E_{+}+i E=-i \mathcal{T},
$$

where $\Delta_{E}$ is the Jordan determinant ([FK94] $)$. Recall the relations

$$
\Delta_{E}(g \cdot x)=\operatorname{det}_{E}(g)^{r / n} \Delta_{E}(x) \quad \text { and } \quad \Delta_{E}(P(y) x)=\Delta_{E}(y)^{2} \Delta_{E}(x)
$$

([FK94 Prop. III.4.2]). For the corresponding one-dimensional representation $\left(\rho_{s}, \mathbb{C}\right)$ of $\widetilde{H}$, the relations

$$
\Delta_{E}(z)^{-s}=P_{\rho_{s}}(z)=\rho_{s}(\widetilde{P}(z)) \quad \text { and } \quad \widetilde{P}(g . z)=g \widetilde{P}(z) g^{*}
$$

now lead to $\rho_{s}\left(g g^{*}\right)=\operatorname{det}_{E}(g)^{-r s / n}$, which in turn shows that

$$
\rho(g)=\operatorname{det}_{E}(g)^{-r s / 2 n} .
$$

It follows in particular that, as a representation of $\widetilde{H}, \rho$ actually factors through the group $H$ itself. 


\subsection{Distribution vectors}

The main purpose of this section is to realize $C$-positive unitary representations of $\widetilde{G}$ in such a way that we get a nice picture of a sufficiently large space of distribution vectors. This is finally done in this subsection, where we formulate the precise results on distribution vectors that we shall use in Section 5 to construct local nets of standard subspaces. The distribution vectors are most easily identified in the $L^{2}$-realization.

Proposition 4.13. For $\xi \in \mathcal{K}$, the corresponding constant function on $E^{*}$ defines a distribution vector $\eta_{\xi}$ of the unitary representation of $(E,+)$ on $L^{2}\left(E^{*}, \mu ; \mathcal{K}\right)$ by

$$
\eta_{\xi}(f)=\int_{E^{*}}\langle d \mu(\lambda) f, \xi\rangle .
$$

These distribution vectors have the following properties:

(a) The subspace $\left\{\eta_{\xi}: \xi \in \mathcal{K}\right\}$ in $L^{2}\left(E^{*}, \mu ; \mathcal{K}\right)^{-\infty}$ is cyclic for the group $(E,+)$.

(b) $U^{-\infty}(0, g) \eta_{\xi}=\eta_{\rho^{\theta}(g) \xi}$ for $g \in \widetilde{H}$ and $\xi \in \mathcal{K}$.

(c) $U^{-\infty}(0, \tau) \eta_{\xi}=\eta_{J_{\mathcal{K}} \xi}$.

Proof. Let $\|\cdot\|$ denote the euclidean norm on $E^{*}$. Since the measure $\mu$ is tempered ([HN01, Thm. V.12]), there exists an $m \in \mathbb{N}$ such that, for every $\xi \in \mathcal{K}$, the integral

$$
\int_{E^{*}} \frac{1}{\left(1+\|\lambda\|^{2}\right)^{2 m}}\langle\xi, \mathrm{d} \mu(\lambda) \xi\rangle
$$

is finite. This implies that $\xi \in\left(1+\|\lambda\|^{2}\right)^{m} L^{2}\left(E^{*}, \mu ; \mathcal{K}\right) \subseteq L^{2}\left(E^{*}, \mu ; \mathcal{K}\right)^{-\infty}$, because the enveloping algebra $S(E)$ of the Lie algebra of the abelian group $(E,+)$ acts on $L^{2}\left(E^{*}, \mu ; \mathcal{K}\right)$ by multiplication with polynomial functions on $E^{*}$.

(a) We first observe that, for $\varphi \in C_{c}^{\infty}(E)$, we have

$$
(U(\varphi) f)(\lambda)=\int_{E} \varphi(x) e^{-i \lambda(x) / 2} f(\lambda) d \mu_{E}(x)=\widehat{\varphi}\left(\frac{\lambda}{2}\right) f(\lambda), \quad \text { where } \quad \widehat{\varphi}(\lambda):=\int_{E} \varphi(x) e^{-i \lambda(x)} d \mu_{E}(x)
$$

is the Fourier transform of $\varphi$. As $\mathcal{S}\left(E^{*}\right) \cdot \mathcal{K} \subseteq \mathcal{S}\left(E^{*}, \mathcal{K}\right)$ is dense and $\mathcal{S}\left(E^{*}, \mathcal{K}\right)$ maps to a dense subspace of $L^{2}\left(E^{*}, \mu ; \mathcal{K}\right)$, the assertion follows.

(b) For $g \in \widetilde{H}$, we have

$$
\begin{aligned}
\eta_{\rho(g)^{*} \xi}(f) & =\left\langle f, \rho(g)^{*} \xi\right\rangle=\int_{E^{*}}\left\langle d \mu(\lambda) f(\lambda), \rho(g)^{*} \xi\right\rangle=\int_{E^{*}}\left\langle f(\lambda), d \mu(\lambda) \rho(g)^{*} \xi\right\rangle \\
& =\int_{E^{*}}\left\langle\rho\left(g^{-1}\right)^{*} f(\lambda), \rho(g) d \mu(\lambda) \rho(g)^{*} \xi\right\rangle \\
& =\int_{E^{*}}\left\langle\rho\left(g^{-1}\right)^{*} f\left(g^{-1} \cdot \lambda\right), d \mu(\lambda) \xi\right\rangle \\
& =\int_{E^{*}}\left\langle\rho^{\theta}(g) f\left(g^{-1} \cdot \lambda\right), d \mu(\lambda) \xi\right\rangle=\int_{E^{*}}\langle(U(0, g) f)(\lambda), d \mu(\lambda) \xi\rangle \\
& =\eta_{\xi}(U(0, g) f)=\left(U^{-\infty}\left(0, g^{-1}\right) \eta_{\xi}\right)(f) .
\end{aligned}
$$

This implies (b).

(c) This assertion follows from

$$
\begin{aligned}
\eta_{J_{\mathcal{K}} \xi}(f) & =\int_{E^{*}}\left\langle d \mu(\lambda) f(\lambda), J_{\mathcal{K}} \xi\right\rangle=\int_{E^{*}}\left\langle f(\lambda), d \mu(\lambda) J_{\mathcal{K}} \xi\right\rangle \\
& =\int_{E^{*}}\left\langle f(\lambda), J_{\mathcal{K}} d \mu(\lambda) \xi\right\rangle=\int_{E^{*}}\left\langle d \mu(\lambda) \xi, J_{\mathcal{K}} f(\lambda)\right\rangle \\
& =\overline{\eta_{\xi}(U(0, \tau) f)}=\left(U^{-\infty}(0, \tau) \eta_{\xi}\right)(f) .
\end{aligned}
$$


For later use, we collect some information on the boundary values of the holomorphic functions in $\mathcal{H}_{\rho} \subseteq \operatorname{Hol}(\mathcal{T}, \mathcal{K})$.

Lemma 4.14. For $\varphi \in C_{c}^{\infty}(E)$ and $F \in \mathcal{H}_{\rho}$, we consider the holomorphic function

$$
F_{\varphi}=U\left(\varphi^{*}\right) F: \mathcal{T} \rightarrow \mathcal{K}, \quad F_{\varphi}(z):=\int_{E} \overline{\varphi(x)} F(z+x) d \mu_{E}(x),
$$

where $d x=d \mu_{E}(x)$ denotes a Haar measure on $E$. This function extends to a continuous function on $\overline{\mathcal{T}}=E+i \overline{E_{+}}$which is smooth on $E$.

Proof. We write $F=\Phi(f)$ for some $f \in L^{2}\left(E^{*}, \mu ; \mathcal{K}\right)$ as in 63). Then the integrated representation of the convolution algebra $C_{c}^{\infty}(E)$ on $L^{2}\left(E^{*}, \mu ; \mathcal{K}\right)$ is given by

$$
(U(\varphi) f)(\lambda)=\int_{E} \varphi(x) e^{-i \lambda(x) / 2} f(\lambda) d \mu_{E}(x)=\widehat{\varphi}(\lambda / 2) f(\lambda) .
$$

We thus obtain

$$
\begin{aligned}
F_{\varphi}(z) & \left.=\int_{E} \varphi^{*}(x) F(z-x) d \mu_{E}(x)=\left(U_{\rho}\left(\varphi^{*}\right) F\right)\right)(z)=\left(U_{\rho}\left(\varphi^{*}\right) \Phi(f)\right)(z) \\
& =\left(\Phi\left(U\left(\varphi^{*}\right) f\right)\right)(z)=\int_{E^{*}} e^{i \lambda(z) / 2} d \mu(\lambda) \overline{\widehat{\varphi}(\lambda / 2)} f(\lambda) .
\end{aligned}
$$

Next we observe that the function $\overline{\widehat{\varphi}(\lambda / 2)} f(\lambda)$ on $E^{*}$ is $\mu$-integrable because $\widehat{\varphi}$ is a Schwartz function, hence $L^{2}$. As

$$
\left|e^{i \lambda(z) / 2}\right|=e^{-\operatorname{Im} \lambda(z) / 2}=e^{-\lambda(\operatorname{Im} z) / 2} \leq 1 \quad \text { for } \quad z \in \overline{\mathcal{T}}
$$

it follows from the Dominated Convergence Theorem that $F_{\varphi}$ extends to a continuous function on $\overline{\mathcal{T}}$. Its restriction to $E$ is the Fourier transform of the measure $d \mu(\lambda) \overline{\widehat{\varphi}(\lambda / 2)} f(\lambda)$, which remains finite when multiplied by any polynomial. Therefore its Fourier transform is smooth.

Remark 4.15. (a) The preceding lemma implies in particular that $\operatorname{bd}(F)(\varphi):=F_{\varphi}(0)$ is defined for every $F \in \mathcal{H}_{\rho}$ and $\varphi \in C_{c}^{\infty}(E)$. To see that this defines a tempered distribution on $E$, we note that, for $F=\Phi(f), f \in L^{2}\left(E^{*}, \mu ; \mathcal{K}\right)$, we have

$$
F_{\varphi}(0)=\int_{E^{*}} \overline{\widehat{\varphi}(\lambda / 2)} d \mu(\lambda) f(\lambda)
$$

and that the measure $\mu \cdot f$ on $E^{*}$ is tempered. Therefore the continuity of the Fourier transform $\mathcal{S}(E) \rightarrow \mathcal{S}\left(E^{*}\right)$ implies that $\mathrm{bd}(F)$ defines a $\mathcal{K}$-valued tempered distribution on $E$. This leads to an injective linear map

$$
\mathrm{bd}: \mathcal{H}_{\rho} \rightarrow \mathcal{S}^{\prime}(E, \mathcal{K}), \quad \operatorname{bd}(F)(\varphi)=F_{\varphi}(0),
$$

whose image is a Hilbert space of tempered distributions on which the scalar product is defined by the requirement that bd is isometric.

(b) To identify this Hilbert space as a reproducing kernel Hilbert space, we write

$$
\operatorname{ev}_{\varphi}: \operatorname{bd}\left(\mathcal{H}_{\rho}\right) \rightarrow \mathcal{K}
$$

for evaluation in $\varphi$, which is a linear map, but antilinear in $\varphi$. For $F \in \mathcal{H}_{\rho}$, we then have

$$
\begin{aligned}
\left\langle\mathrm{bd}^{*} \operatorname{ev}_{\varphi}^{*} \xi, F\right\rangle & =\left\langle\xi, F_{\varphi}(0)\right\rangle=\int_{E^{*}} \overline{\widehat{\varphi}(\lambda / 2)}\left\langle\xi, d \mu(\lambda) \Phi^{-1}(F)(\lambda)\right\rangle \\
& =\left\langle\widehat{\varphi}(\cdot / 2) \xi, \Phi^{-1}(F)\right\rangle_{L^{2}}=\langle\Phi(\widehat{\varphi}(\cdot / 2) \xi), F\rangle_{\mathcal{H}_{\rho}} .
\end{aligned}
$$


We conclude that

$$
\mathrm{bd}^{*} \operatorname{ev}_{\varphi}^{*} \xi=\Phi(\widehat{\varphi}(\cdot / 2) \xi) \in \mathcal{H}_{\rho} .
$$

The corresponding reproducing kernel is therefore given by

$$
\left\langle\xi, \operatorname{ev}_{\varphi} \operatorname{ev}_{\psi}^{*} \eta\right\rangle=\left\langle\mathrm{bd}^{*} \operatorname{ev}_{\varphi}^{*} \xi, \mathrm{bd}^{*} \operatorname{ev}_{\psi}^{*} \eta\right\rangle=\langle\widehat{\varphi}(\cdot / 2) \xi, \widehat{\psi}(\cdot / 2) \eta\rangle_{L^{2}}=\int_{E^{*}} \overline{\widehat{\varphi}(\lambda / 2)} \widehat{\psi}(\lambda / 2)\langle\xi, d \mu(\lambda) \eta\rangle .
$$

Next we note that the holomorphic function

$$
D: \mathcal{T} \rightarrow B(\mathcal{K}), \quad D(z)=\int_{E^{*}} e^{i \lambda(z) / 2} d \mu(\lambda)
$$

has distributional boundary values $D \in \mathcal{S}^{\prime}(E, B(\mathcal{K}))$, given by

$$
D(\varphi)=\int_{E^{*}} \widetilde{\bar{\varphi}}(\lambda / 2) d \mu(\lambda)=\int_{E^{*}} \overline{\widehat{\varphi}(\lambda / 2)} d \mu(\lambda)
$$

(ÓO20, Lemma 3.22]). This distribution satisfies

$$
D\left(\psi^{*} * \varphi\right)=\int_{E^{*}} \overline{\widehat{\varphi}(\lambda / 2)} \widehat{\psi}(\lambda / 2) d \mu(\lambda)=\operatorname{ev}_{\varphi} \operatorname{ev}_{\psi}^{*},
$$

so that it represents the reproducing kernel of the subspace $\operatorname{bd}\left(\mathcal{H}_{\rho}\right) \subseteq \mathcal{S}^{\prime}(E, \mathcal{K}$ ) (cf. NÓ18, Def. 7.1.5], which does not have the factor $\frac{1}{2}$ ).

\section{$5 \quad$ Nets of standard subspaces}

Let $\mathfrak{g}$ be a direct sum of simple hermitian Lie algebras of tube type (see the table in the introduction for a list of these Lie algebras) and $(\rho, \mathcal{K})$ be a $\mathcal{T}$-positive norm-continuous representation of $\widetilde{H}_{\mathbb{C}, \tau}$ (cf. Remark 4.10), so that we obtain an antiunitary representation $\left(U_{\rho}, \mathcal{H}_{\rho}\right)$ of $\widetilde{G}_{\tau}$ on the Hilbert subspace $\mathcal{H}_{\rho} \subseteq \operatorname{Hol}(\mathcal{T}, \mathcal{K})$. In this section we show that, for the standard subspace $\mathrm{V} \subseteq \mathcal{H}_{\rho}$ specified by the pair $\left(h, \tau_{G}\right)$, there exists a real subspace $\mathrm{E} \subseteq \mathcal{K}$ contained in $\mathrm{V}^{-\infty}$, to which we can apply Section 3. This leads to the identity $\mathrm{V}=\mathrm{H}_{\mathrm{E}}\left(S_{\mathrm{V}}^{0}\right)$ and to a net $\mathrm{V}(\mathcal{O}):=\mathrm{H}_{\mathrm{E}}(\mathcal{O})$ of cyclic subspaces associated to non-empty open subsets $\mathcal{O} \subseteq G$. As a consequence of the construction in Section 4 this covers all irreducible $C$-positive antiunitary representations $(U, \mathcal{H})$ of $\widetilde{G}_{\tau}$ (Theorem 5.1).

\subsection{Nets of standard subspaces on Lie groups}

We briefly recall our setting. In this section $\mathfrak{g}$ denotes a semisimple Lie algebra.

- We assume that $\mathfrak{g}$ contains a closed pointed generating invariant convex cone $C \subseteq \mathfrak{g}$.

- We fix an Euler element $0 \neq h \in[\mathfrak{g}, \mathfrak{g}]$, i.e., $\operatorname{ad} h$ is diagonalizable with $\operatorname{Spec}(\operatorname{ad} h) \subseteq$ $\{-1,0,1\}$. All these elements are conjugate under inner automorphisms (cf. MN20. Ex. 6.1]), so that it suffices to consider one such element $h$. Together with the preceding item, the existence of such an element is equivalent to all simple non-compact ideals of $\mathfrak{g}$ being hermitian of tube type (see the proof of [MN20, Thm 3.12] and [Ó91 Thm. 5.6]).

- We assume that $\mathfrak{g}^{0}(h)=\operatorname{ker}(\operatorname{ad} h)$ contains no proper ideal, i.e., that $\mathfrak{g}$ is generated as a Lie algebra by the eigenspaces $\mathfrak{g}^{ \pm 1}(h)$. This excludes compact ideals in $\mathfrak{g}$.

- We consider on $\mathfrak{g}$ the involution $\tau_{h}:=e^{\pi i \text { ad } h}$ fixing $h$.

- Then the eigenspace $E:=\mathfrak{g}^{1}=\mathfrak{g}^{1}(h)$ is a unital euclidean Jordan algebra, where the unit element $e \in E$ can be chose such that the open cone $E_{+}$of positive squares satisfies

$$
\overline{E_{+}}=E \cap C=\mathfrak{g}^{1}(h) \cap C=: C_{+} .
$$


As in Section 4, we consider the connected Lie group $G:=\operatorname{Aut}(\mathfrak{g})_{0}$ and write $q_{G}: \widetilde{G} \rightarrow G$ for its universal covering group. Let $\tau_{G} \in \operatorname{Aut}(\widetilde{G})$ be the involutive automorphism integrating $\tau_{h} \in \operatorname{Aut}(\mathfrak{g})$. Then

$$
\widetilde{G}_{\tau}:=\widetilde{G} \rtimes\left\{\operatorname{id}_{G}, \tau\right\}
$$

is a graded Lie group with two connected components. We write

$$
\mathfrak{h}=\mathfrak{g}^{0}=\operatorname{ker}(\operatorname{ad} h), \quad H:=\left\langle\exp _{G} \mathfrak{h}\right\rangle \subseteq G, \quad \text { and } \quad H_{\tau}:=H \times\{\operatorname{id}, \tau\} \subseteq G_{\tau} .
$$

We also note that

$$
\widetilde{G}^{\tau_{G}}=\left\{g \in \widetilde{G}: \tau_{G}(g)=g\right\}
$$

is connected because $\widetilde{G}$ is simply connected ([Lo69, Thm. IV.3.4]), so that $q_{G}$ restricts to a covering map

$$
q_{H}: \widetilde{G}^{\tau_{G}} \rightarrow H
$$

Theorem 5.1. (Realization Theorem) For each irreducible antiunitary representation $(U, \mathcal{H})$ of $\widetilde{G}_{\tau}$ for which $C_{U}$ is pointed and generating, there exists an irreducible involutive finite dimensional representation $(\rho, \mathcal{K})$ of the product group

$$
\widetilde{H}_{\tau}:=\widetilde{H} \times\{\mathbf{1}, \tau\}
$$

with the following properties:

(a) The holomorphic extension of $\rho$ to the universal complexification $\widetilde{H}_{\mathbb{C}}$ is $\mathcal{T}$-positive, i.e., the $B(\mathcal{K})$-valued kernel

$$
Q_{\rho}(z, w):=P_{\rho}\left(\frac{z-\bar{w}}{2 i}\right)
$$

on the tube domain $\mathcal{T}=E+i E_{+}$is positive definite.

(b) $(U, \mathcal{H})$ is equivalent to the representation of $\widetilde{G}_{\tau}$ on the corresponding reproducing kernel Hilbert space $\mathcal{H}_{\rho} \subseteq \operatorname{Hol}(\mathcal{T}, \mathcal{K})$, given by

$$
\left(U_{\rho}(g) f\right)(z)=J_{\rho}\left(g, g^{-1} . z\right) f\left(g^{-1} . z\right) \quad \text { for } \quad g \in \widetilde{G}, z \in \mathcal{T},
$$

and

$$
\left(U_{\rho}\left(\tau_{G}\right) f\right)(z):=J_{\mathcal{K}} f(-\bar{z}),
$$

where $J_{\mathcal{K}}=\rho(\tau), \widetilde{J}: \widetilde{G} \times \mathcal{T} \rightarrow \widetilde{H}_{\mathbb{C}}$ is the lift of the cocycle $J: G \times \mathcal{T} \rightarrow H_{\mathbb{C}}, J(g, z):=\mathrm{d} g(z)$, and $J_{\rho}:=\rho \circ \widetilde{J}$.

Proof. Recall the isomorphism $\widetilde{\alpha}_{c}: \widetilde{G}(\mathcal{D})_{\tau} \rightarrow \widetilde{G}_{\tau}=\widetilde{G}(\mathcal{T})_{\tau}$ from (44). Composing with $\widetilde{\alpha}_{c}$, any irreducible antiunitary representation $(U, \mathcal{H})$ of $\widetilde{G}_{\tau}$ for which $C_{U}$ is pointed and generating defines a representation $\left(U^{c}, \mathcal{H}\right)$ of $\widetilde{G}(\mathcal{D})_{\tau}$ with the same property. From [Ne99, Thm. X.3.9] we know that this is a highest weight representation for a suitably chosen positive system, hence can be realized in the $\operatorname{space} \operatorname{Hol}(\mathcal{D}, \mathcal{K})$ of holomorphic functions on $\mathcal{D}$, where $\mathcal{K}$ carries an irreducible antiunitary representation $\left(\rho^{c}, \mathcal{K}\right)$ of the simply connected group $\widetilde{K}(\mathcal{D})_{\tau}$ (Proposition 4.6, Ne99, Thm. XII.2.6]). We conclude that the kernel $Q_{\rho}^{\mathcal{D}}:=\rho \circ \widetilde{Q}^{\mathcal{D}}$ is positive definite (Ne99, Prop. XII.2.1]), Now Lemma 4.4 transports all this structure from $\mathcal{D}$ to $\mathcal{T}$, so that we obtain the required realization in a Hilbert space $\mathcal{H}_{\rho} \subseteq \operatorname{Hol}(\mathcal{T}, \mathcal{K})$, where $(\rho, \mathcal{K})$ is a finite-dimensional $*$-representation of $\widetilde{H}_{\tau}$, and the representation is given by (69) and (70).

We now turn to the standard subspace $\mathrm{V}=\mathrm{V}_{\left(h, \tau_{G}, U\right)}$ specified by the multiplicative oneparameter group of $G_{\tau}$, corresponding to the pair $\left(h, \tau_{G}\right)$ via

$$
J_{\mathrm{V}}=U\left(\tau_{G}\right) \quad \text { and } \quad \Delta_{\mathrm{V}}^{-i t / 2 \pi}=U(\exp t h) \quad \text { for } \quad t \in \mathbb{R} .
$$


In the following proposition, we shall see in particular that the distribution vectors $\Phi\left(\eta_{\xi}\right) \in$ $\mathcal{H}_{\rho}^{-\infty}$ are invariant under the subgroup $N^{-}=\exp \left(\mathfrak{g}^{-1}(h)\right) \subseteq \widetilde{G}(\mathcal{T})$. As the action of this subgroup is not so easily accessible in the $L^{2}$-picture, we study this problem in the holomorphic picture on $\mathcal{H}_{\rho}$.

Proposition 5.2. Let $(\rho, \mathcal{K})$ be a holomorphic $\mathcal{T}$-positive *-representation of $\widetilde{H}_{\mathbb{C}}$ and let $\mathrm{ev}_{0}: \mathcal{H}_{\rho}^{\infty} \rightarrow \mathcal{K}, F \mapsto F(0)$ be the evaluation in $0 \in \overline{\mathcal{T}}$. Then the following assertions hold:

(a) For each $\xi \in \mathcal{K}$, we obtain a distribution vector $\mathrm{ev}_{0}^{\xi}: \mathcal{H}_{\rho}^{\infty} \rightarrow \mathbb{C}, \mathrm{ev}_{0}^{\xi}(F):=\langle F(0), \xi\rangle$.

(b) $U_{\rho}(g) \mathrm{ev}_{0}^{\xi}=\operatorname{ev}_{0}^{\rho(\bar{\theta}(\widetilde{J}(g))) \xi}$ for $g \in \widetilde{G}^{\tau_{G}} \subseteq \widetilde{G}$ and $\widetilde{J}(g) \in \widetilde{H}_{\mathbb{C}}$.

(c) $\operatorname{ev}_{0}^{\xi}$ is invariant under the subgroup $N^{-}=\exp \left(\mathfrak{g}^{-1}(h)\right) \subseteq \widetilde{G}=\widetilde{G}(\mathcal{T})$.

(d) The real subspace

$$
\mathrm{E}:=\left\{\operatorname{ev}_{0}^{\xi}: e^{-\pi i \cdot \mathrm{d} \rho(h)} \xi=J_{\mathcal{K}} \xi\right\}
$$

is contained in $\mathcal{H}_{\rho, \mathrm{ext}, J}^{-\infty}$ and invariant under the subgroup $P^{-}:=N^{-} \widetilde{G}^{\tau_{G}}$.

Proof. (a) Let $F \in \mathcal{H}_{\rho}$ be a smooth vector. Then it is in particular smooth for the representation of $(E,+) \cong \exp \left(\mathfrak{g}^{1}(h)\right)$. By the Dixmier-Malliavin Theorem (DM78]), it is of the form $F=U_{\rho}(\varphi) F_{0}$ for some $\varphi \in C_{c}^{\infty}(E)$ and $F_{0} \in \mathcal{H}_{\rho}$. Then

$$
F(z)=\int_{E} \varphi(x) F_{0}(z-x) d \mu_{E}(x)
$$

so that Lemma 4.14 implies that $F$ extends to a continuous function on $\overline{\mathcal{T}}$ which is smooth on $E$. Therefore evo is defined.

Next we note that, for $F=\Phi(f)$ and $f \in L^{2}\left(E^{*}, \mu ; \mathcal{K}\right)^{\infty}$,

$$
\begin{aligned}
\Phi\left(\eta_{\xi}\right)(F) & =\Phi\left(\eta_{\xi}\right)(\Phi(f))=\eta_{\xi}(f)=\int_{E^{*}}\langle f(\lambda), d \mu(\lambda) \xi\rangle \\
& =\int_{E^{*}}\langle d \mu(\lambda) f(\lambda), \xi\rangle=\langle\Phi(f)(0), \xi\rangle=\langle F(0), \xi\rangle=\operatorname{ev}_{0}^{\xi}(F) .
\end{aligned}
$$

As $\eta_{\xi}$ is a distribution vector for the representation of $\left(E,_{+}\right)$on $L^{2}\left(E^{*}, \mu ; \mathcal{K}\right)$, the same holds for $\mathrm{ev}_{0}^{\xi}$ and the representation of the translation group $(E,+)$ on $\mathcal{H}_{\rho}$.

(b) follows from

$$
\begin{aligned}
\left(U_{\rho}^{-\infty}(g) \operatorname{ev}_{0}^{\xi}\right)(F) & =\operatorname{ev}_{0}^{\xi}\left(U_{\rho}^{\infty}\left(g^{-1}\right) F\right)=\left\langle\left(U_{\rho}^{\infty}\left(g^{-1}\right) F\right)(0), \xi\right\rangle \\
& =\left\langle\rho\left(g^{-1}\right) F(0), \xi\right\rangle=\left\langle F(0), \rho\left(g^{-1}\right)^{*} \xi\right\rangle=\langle F(0), \rho(\theta(g)) \xi\rangle .
\end{aligned}
$$

(c) For $g \in N^{-}$, we have $U_{\rho}(g) F \in \mathcal{H}_{\rho}^{\infty}$ and

$$
\left(U_{\rho}(g) F\right)(0)=\lim _{z \rightarrow 0}\left(U_{\rho}(g) F\right)(z)=\lim _{z \rightarrow 0} J_{\rho}\left(g, g^{-1} . z\right) F\left(g^{-1} . z\right)=J_{\rho}\left(g, g^{-1} .0\right) F\left(g^{-1} .0\right)=F(0)
$$

because $\mathrm{d} g(0)=\mathbf{1}$ for every $g \in N^{-}$. This implies that the distribution vectors $\operatorname{ev}_{0}^{\xi}$ are $N^{-}$-invariant.

(d) On $\mathcal{T} \subseteq E_{\mathbb{C}}$, the one-parameter group $\exp (\mathbb{R} h)$ acts by $\exp (t h) z=e^{t} z$. Then

$$
U^{-\infty}(\exp t h) \mathrm{ev}_{0}^{\xi}=\operatorname{ev}_{0}^{\rho(\exp -t h) \xi} \quad \text { for every } \quad \xi \in \mathcal{K}
$$

follows from (b). This shows that $\mathrm{ev}_{0}^{\xi} \in \mathcal{H}_{\rho, \text { ext }, J}^{-\infty}$ if and only if $e^{-\pi i \mathrm{~d} \rho(h)} \xi=J_{\mathcal{K}} \xi$. As $J_{\mathcal{K}}$ and $\mathrm{d} \rho(h)$ commute with $\rho(\widetilde{H})$, the real subspace $\mathrm{E}$ is $U\left(\widetilde{G}^{\tau_{G}}\right)$-invariant. From (c) we have the invariance of each $\operatorname{ev}_{0}^{\xi}$ under the subgroup $N^{-}$, so that $\mathrm{E}$ is invariant under $P^{-}=N^{-} \widetilde{G}^{\tau_{G}}$.

We are now ready to prove the main theorem of this paper. 
Theorem 5.3. For a holomorphic $\mathcal{T}$-positive *-representation $(\rho, \mathcal{K})$ of $\widetilde{H}_{\mathbb{C}}$, we consider the irreducible antiunitary representation $\left(U_{\rho}, \mathcal{H}_{\rho}\right)$ of $\widetilde{G}_{\tau}$ from (69) and (70) and the real subspace $\mathrm{E} \subseteq \mathcal{H}_{\rho}^{-\infty}$ from (72). Then the following assertions hold:

(a) The standard subspace $\mathrm{V}$ of $\mathcal{H}_{\rho}$, specified by the conjugation $J_{\mathrm{V}}=U_{\rho}\left(\tau_{G}\right)$ and the modular group $\Delta_{\mathrm{V}}^{-i t / 2 \pi}=U_{\rho}(\exp t h)$, coincides with

$$
\mathrm{H}_{\mathrm{E}}(S)=\overline{\operatorname{span}_{\mathbb{R}}\left(U^{-\infty}\left(C_{c}^{\infty}(S, \mathbb{R})\right) \mathrm{E}\right)}, \quad \text { where } \quad S:=\widetilde{G}^{\tau_{G}} \exp \left(C_{+}^{0} \oplus C_{-}^{0}\right)
$$

is an open connected subsemigroup of $G$.

(b) The open subsemigroup $S_{\mathrm{V}}^{0} \supseteq S$ also satisfies $\mathrm{H}_{\mathrm{E}}\left(S_{\mathrm{V}}^{0}\right)=\mathrm{V}$.

(c) The prescription $\mathrm{V}(\mathcal{O}):=\mathrm{H}_{\mathrm{E}}(\mathcal{O})$ assigns to every non-empty open subset $\mathcal{O} \subseteq G$ a real cyclic subspace, and this net has the following properties:

(I) Isotony: $\mathcal{O}_{1} \subseteq \mathcal{O}_{2}$ implies $\mathrm{V}\left(\mathcal{O}_{1}\right) \subseteq \mathrm{V}\left(\mathcal{O}_{2}\right)$.

(Cov) Covariance: $\mathrm{V}(g \mathcal{O})=U(g) \mathrm{V}(\mathcal{O})$ for $g \in G$.

(BW) Bisognano-Wichmann property: For $g \in G$ and $\mathcal{W}:=g S$, the subspace $\mathrm{V}(\mathcal{W})$ is standard with

$$
\Delta_{\mathrm{v}(\mathcal{W})}^{-i t / 2 \pi}=U(\exp t \operatorname{Ad}(g) h) \quad \text { for } \quad t \in \mathbb{R}, g \in G \quad \text { and } \quad J_{\mathrm{v}(\mathcal{W})}=U\left(g \tau_{G}(g)^{-1}\right) J_{\mathrm{V}} .
$$

(Inv) $\mathrm{V}(g S)^{\prime}=\mathrm{V}\left(g S^{-1}\right)=\mathrm{V}\left(g \tau_{G}(S)\right)$ for $g \in G$.

(d) If $\emptyset \neq \mathcal{O} \subseteq G$ is open and there exists a $g \in G$ with $\mathcal{O} \subseteq g S$, then $\mathrm{V}(\mathcal{O})$ is standard.

(e) If $A \mathcal{O} S \subseteq \mathcal{O} \supseteq S$ and $\mathrm{V}(\mathcal{O})$ is standard, then $\mathcal{O} \subseteq S_{\mathrm{V}}^{0}$. In particular

- If $g \in S^{-1} \backslash S_{\mathrm{v}}$, then $\mathrm{V}(A g S)$ is not standard.

- $\mathrm{V}(G)$ is not standard.

Proof. (a) From Proposition [5.2(d) and Lemma 3.12 it follows that $\mathrm{E} \subseteq \mathcal{H}_{\rho, \text { ext }, J}^{-\infty} \subseteq \mathrm{V}^{-\infty}$.

As $S_{\mathrm{V}}^{0}=G_{\mathrm{V}} \exp \left(C_{+}^{0} \oplus C_{-}^{0}\right)$ by (6) in the introduction, and $\widetilde{G}^{\tau_{G}}=\left(G_{\mathrm{V}}\right)_{0}$, we have $S_{\mathrm{V}}^{0}=G_{\mathrm{V}} S$ and in particular $S \subseteq S_{\mathrm{v}}$. For $\varphi \in C_{c}^{\infty}(S, \mathbb{R})$, Lemma 3.3 (c) implies $U^{-\infty}(\varphi) \mathrm{E} \subseteq \mathrm{V}$, so that $\mathrm{H}_{E}(S) \subseteq \mathrm{V}$. As V is standard, $\mathrm{H}_{\mathrm{E}}(S)$ is also separating, hence standard. The invariance of $\mathrm{E}$ under $U^{-\infty}(A)$ and the invariance of $S$ under conjugation with $A$ show that $\mathrm{H}_{\mathrm{E}}(S)$ is invariant under $\Delta_{\mathrm{V}}^{i \mathbb{R}}=U(A)$. Now $\mathrm{H}_{\mathrm{E}}(S)=\mathrm{V}$ follows from Lemma 3.4

(b) From (a) we recall that $S_{\mathrm{V}}^{0}=G_{\mathrm{V}} S$. By (a), $\mathrm{V}=\mathrm{H}_{\mathrm{E}}(S)$, and this subspace is invariant under $G_{\mathrm{V}}$, so that Lemma 2.11 shows that $\mathrm{H}_{\mathrm{E}}(S)=\mathrm{H}_{\mathrm{E}}\left(G_{\mathrm{V}} S\right)=\mathrm{H}\left(S_{\mathrm{V}}^{0}\right)$.

(c) Since $\mathrm{E} \subseteq \mathcal{H}^{-\infty}$ is $G$-cyclic in the sense that $\mathcal{H}_{\mathrm{E}}(G)=\mathcal{H}$ (Proposition 4.13 (a)), Theorem 3.5 implies that each subspace $\mathrm{H}_{\mathrm{E}}(\mathcal{O}), \mathcal{O} \neq \emptyset$, is cyclic. Properties (I) and (Cov) are trivial, as we have already observed in Definition 2.9. Property (BW) follows from (a) and the covariance of the BGL net.

To verify (Inv), we first note that, replacing $h$ by $-h$ corresponds to replacing V by V' (Lo08, Prop. 3.3]) and $S=\widetilde{G}^{\tau_{G}} \exp \left(C_{+}^{0}+C_{-}^{-}\right)$by $S^{-1}=\widetilde{G}^{\tau_{G}} \exp \left(-C_{+}^{0}-C_{-}^{0}\right)$. Therefore (a) implies $\mathrm{V}^{\prime}=\mathrm{H}_{\mathrm{E}}\left(S^{-1}\right)=\mathrm{V}\left(S^{-1}\right)$. For $g \in G$, we thus obtain

$$
\mathrm{V}(g S)^{\prime}=(U(g) \mathrm{V})^{\prime}=U(g) \mathrm{V}^{\prime}=U(g) \mathrm{V}\left(S^{-1}\right)=\mathrm{V}\left(g S^{-1}\right) .
$$

Finally, we note that $\mathbf{L}\left(\tau_{G}\right)\left(C_{ \pm}\right)=-C_{ \pm}$and $\tau_{G}\left(\widetilde{G}^{\tau_{G}}\right)=\widetilde{G}^{\tau_{G}}$ implies $\tau_{G}(S)=S^{-1}$.

(d) By (Cov) we have $\mathrm{V}(\mathcal{O}) \subseteq U(g) \mathrm{V}(S)$, and the right hand side is standard. Hence the cyclic subspace $\mathrm{V}(\mathcal{O})$ is also separating and therefore standard.

(e) As $A \mathcal{O}=\mathcal{O} \supseteq S$, (Cov) implies that the real subspace, $\mathrm{V}(\mathcal{O})$ is invariant under the modular group $U(A)$ of $\mathrm{V}=\mathrm{V}(S) \subseteq \mathrm{V}(\mathcal{O})$. If $\mathrm{V}(\mathcal{O})$ is standard, then Lemma 3.4 implies that $\mathrm{V}(\mathcal{O})=\mathrm{V}$. This in turn shows that, for $g \in \mathcal{O}$, we have

$$
U(g) \mathrm{V}=U(g) \mathrm{V}(S)=\mathrm{V}(g S) \subseteq \mathrm{V}(\mathcal{O})=\mathrm{V} .
$$


We conclude that $\mathcal{O} \subseteq S_{\mathrm{v}}$, and since $\mathcal{O}$ is open, that $\mathcal{O} \subseteq S_{\mathrm{V}}^{0}$.

As $S_{\mathrm{V}} \neq G$, it follows in particular that $\mathrm{H}_{\mathrm{E}}(G)$ is not standard. For $g \in S^{-1} \backslash S_{\mathrm{V}}$, the relation $g^{-1} S \subseteq S$ implies that $S \subseteq g S \subseteq A g S=: \mathcal{O}$. Hence $\mathcal{O}$ satisfies the assumptions from above. If $\mathcal{O} \subseteq S_{\mathrm{V}}^{0}$, then $e \in \bar{S}$ leads to the contradiction $g \in S_{\mathrm{v}}$. Therefore $\mathrm{V}(\mathcal{O})$ is not standard.

The preceding theorem provides for every $\mathcal{T}$-positive holomorphic representation $(\rho, \mathcal{K})$ of $\widetilde{H}_{\mathbb{C}, \tau}$, or, equivalently, any norm-continuous real representation $\left(\rho, \mathcal{K}_{\mathbb{R}}\right)$ of $\widetilde{G}^{\tau_{G}}$, a net of cyclic real subspaces, some of which are standard. Applying second quantization functors, we thus obtain free quantum fields in the sense of Haag-Kastler on the group $\widetilde{G}$, where the left translates $\mathcal{W}=g S$ of the semigroup $S$ play the role of wedge domains. Here the invariant cone $\underset{\widetilde{G}}{C} \mathfrak{g}$ defines a biinvariant causal structure in the sense of a cone field $(g C)_{g \in \widetilde{G}}$ on the group $\widetilde{G}$. For $\mathcal{W}=S$, the modular involution corresponds to the involution $\tau_{G}$ on $\widetilde{G}$ which exchanges $S$ and $S^{-1}$. The modular one-parameter group acts on $S$ by the left translations $\alpha_{t}(s)=(\exp t h) s$. By left translation, we obtain the geometric actions of the modular objects on the domains $g S$.

Remark 5.4. The results of this section extend easily to quasihermitian Lie groups, i.e., where the Lie algebra $\mathfrak{g}$ is of the form $\mathfrak{g}=\mathfrak{g}_{1} \oplus \mathfrak{g}_{2}$, where $\mathfrak{g}_{1}$ is a sum of simple hermitian ideals of tube type and $\mathfrak{g}_{2}$ is a compact Lie algebra. Then $\mathfrak{h}=\mathfrak{h}_{1} \oplus \mathfrak{g}_{2}$, and irreducible representations $(\rho, \mathcal{K})$ of $\widetilde{H} \cong \widetilde{H}_{1} \times \widetilde{G}_{2}$ are tensor products $\rho \cong \rho_{1} \otimes \rho_{2}$, where $\rho$ is $\mathcal{T}$-positive if and only if $\rho_{1}$ has this property.

\subsection{Jordan space-times and causal symmetric spaces}

The real subspace $\mathrm{E}$ in Theorem 5.3 that we used above is also invariant under the closed connected subgroups $P_{1}:=\widetilde{G}^{\tau_{G}}$ and $P_{2}:=N^{-} \widetilde{G}^{\tau_{G}}$ (Proposition [5.2). We thus obtain on the simply connected homogeneous spaces $M_{j}:=\widetilde{G} / P_{j}$ covariant nets of cyclic/standard subspaces as follows. Let $q_{j}: \widetilde{G} \rightarrow M_{j}$ denote the canonical projection and put

$$
\mathrm{V}_{M_{j}}(\mathcal{O}):=\mathrm{V}\left(q_{j}^{-1}(\mathcal{O})\right) \quad \text { for } \quad \emptyset \neq \mathcal{O} \subseteq M_{j}
$$

For these nets isotony and $G$-covariance

$$
\mathrm{V}_{M_{j}}(g \mathcal{O})=U(g) \mathrm{V}_{M_{j}}(\mathcal{O})
$$

are clear. For $\mathcal{W}_{j}:=q_{j}(S)$ it follows from Lemma 2.11 that

$$
\mathrm{V}_{M_{j}}\left(\mathcal{W}_{j}\right)=\mathrm{V}\left(q_{j}^{-1}\left(\mathcal{W}_{j}\right)\right)=\mathrm{V}\left(S P_{j}\right)=\mathrm{V}(S) .
$$

Hence the subspace $\mathrm{V}_{M_{j}}\left(\mathcal{W}_{j}\right)$ is standard and has the Bisognano-Wichmann property. Its modular conjugation is implemented on $M_{j}$ by

$$
\tau_{M_{j}}\left(g P_{j}\right)=\tau_{G}(g) P_{j}
$$

and the modular group by

$$
\alpha_{t}\left(g P_{j}\right)=\exp (t h) g P_{j}=\exp (t h) g \exp (-t h) P_{j} \quad \text { for } \quad g \in G, t \in \mathbb{R} .
$$

Here the manifold $M_{1}=\widetilde{G} / P_{1}$ is a symmetric space of Cayley type (cf. HÓ97) and the manifolds $M_{2}=\widetilde{G} / P_{2}$ coincide with the Jordan space-times described by Günaydin in Gu93 as a natural class of causal manifolds with conformal symmetries, represented by the group $G$. These manifolds also coincide with the simple space-time manifolds classified in MdR07, Thm. 4] by Mack and de Riese. 


\subsection{Affine groups}

The subgroup $P^{+}:=N^{+} \widetilde{G}^{\tau_{G}} \subseteq \widetilde{G}$ acts by affine maps on the Jordan algebra $E \cong \mathfrak{g}^{1}(h)$, where $N^{+} \cong(E,+)$ acts by translations, and $G^{\tau_{G}}$ by linear maps leaving the cone $E_{+}$invariant. For the special case, where $E \cong \mathbb{R}^{1, n-1}$ is $n$-dimensional Minkowski space, $P^{+} \cong$ $E \rtimes \mathbb{R}_{+}^{\times} \operatorname{Spin}_{1, n-1}(\mathbb{R})$ is the Poincaré group extended by dilations. Restricting everything from $\widetilde{G}$ to $P^{+}$, we immediately obtain nets of cyclic/standard subspaces on $E$, which we may consider as an open subset of the homogeneous space $G / P^{-}$. In this case it is of some interest to consider an even smaller group, such as the Lorentz group $\mathrm{SO}_{1, n-1}(\mathbb{R})_{0}$ on $E=\mathbb{R}^{1, n-1}$, which does not necessarily contain the dilations.

A natural setting for this is explored in NÓO20, and we now explain briefly how it connects to the present paper. Let $L \subseteq H$ be a connected subgroup invariant under the Cartan involution $\theta$, and $q_{L}: \widetilde{L} \rightarrow L$ be its universal covering group. We consider a norm-continuous *-representation $(\rho, \mathcal{K})$ of $\widetilde{L}_{\tau}=\widetilde{L} \times\{\mathbf{1}, \tau\}$ and a tempered $\operatorname{Herm}^{+}(\mathcal{K})$-valued measure $\mu$ on $E_{+}^{\star}$ such that

$$
\left(g^{-1}\right)_{*} \mu=\rho(g) \mu \rho(g)^{*} \quad \text { for } \quad g \in \widetilde{L} .
$$

Then the Laplace transform $\mathcal{L}(\mu)$ defines a holomorphic function on $\mathcal{T}$, and

$$
Q_{\rho}(z, w):=\mathcal{L}(\mu)\left(\frac{z-\bar{w}}{2 i}\right)
$$

is a positive definite $B(\mathcal{K})$-valued kernel on $\mathcal{T}$. We thus obtain a unitary representation of $E \rtimes \widetilde{L}_{\tau}$ on the corresponding Hilbert subspace $\mathcal{H}_{\rho} \subseteq \operatorname{Hol}(\mathcal{T}, \mathcal{K})$, given by

$$
\left(U_{\rho}(x, g) f\right)(z):=\rho(g) f\left(g^{-1} \cdot(z-x)\right) \quad \text { for } \quad x \in E, z \in \mathcal{T}, g \in \widetilde{L}_{\tau} .
$$

Now we consider a element $h^{\prime} \in \mathfrak{l}$, the Lie algebra of $L$, for which ad $h^{\prime}$ defines a 3 -grading on $\mathfrak{g}$, and the involution $\tau^{\prime}$ on $E \rtimes \widetilde{L}$ integrating the Lie algebra involution $e^{\pi i \text { ad } h^{\prime}}$ on $E \rtimes \mathfrak{l}$. For $J_{\mathcal{K}}:=\rho\left(\tau^{\prime}\right)$, the real subspace

$$
\mathrm{E}:=\left\{\operatorname{ev}_{0}^{\xi}: e^{-\pi i \cdot \mathrm{d} \rho\left(h^{\prime}\right)} \xi=J_{\mathcal{K}} \xi\right\}
$$

is then contained in $\mathcal{H}_{\rho}^{-\infty}\left(\left.U_{\rho}\right|_{E}\right)$ and invariant under $U_{\rho}\left(\widetilde{L}^{0}\right)$, where $\widetilde{L}^{0} \subseteq \widetilde{L}$ is the centralizer of $h^{\prime}$. Now the standard subspace $\mathrm{V}=\mathrm{V}_{\left(h^{\prime}, \tau^{\prime}, U_{\rho}\right)}$ coincides with $\mathrm{H}_{\mathrm{E}}^{E}(\mathcal{W})$, where $\mathcal{W}$ is the wedge domain

$$
\mathcal{W}=\left(E_{+} \cap E^{1}\left(h^{\prime}\right)\right) \oplus E^{0}\left(h^{\prime}\right) \oplus\left(-E_{+} \cap E^{-1}\left(h^{\prime}\right)\right) .
$$

Using Lemma 2.12, we even get

$$
\mathrm{H}_{\mathrm{E}}^{E}(\mathcal{W})=\mathrm{H}_{\mathrm{E}}^{E \rtimes \widetilde{L}^{0}}\left(\mathcal{W} \rtimes \widetilde{L}^{0}\right)
$$

In the special case where $\left.\operatorname{ad} h\right|_{E}=\operatorname{id}_{E}$, we have $\widetilde{L}^{0}=\widetilde{L}$. For more details on this situation, we refer to NÓO20.

\section{Perspectives}

\subsection{Extension to more general groups}

The results of Sections 2 and 3 work for non-reductive Lie groups satisfying the conditions (B1)-(B4). There are various types of non-reductive groups with this property, such as the extended Jacobi group, discussed in some detail in [Ne19b, Ex. 3.7]. We briefly recall some observations from Ne19b. Its Lie algebra is

$$
\mathfrak{g}=\mathfrak{h} \mathfrak{c s p}\left(\mathbb{R}^{2 n}, \omega\right)=\mathfrak{h} \mathfrak{i} \mathfrak{s}\left(\mathbb{R}^{2 n}, \omega\right) \rtimes \mathfrak{c s p} \mathfrak{s}_{2 n}(\mathbb{R}),
$$


where $\omega$ is the canonical symplectic form on $\mathbb{R}^{2 n}, \mathfrak{h} \mathfrak{e i s}\left(\mathbb{R}^{2 n}, \omega\right)=\mathbb{R} \oplus \mathbb{R}^{2 n}$ is the corresponding Heisenberg algebra with the bracket $\left[(z, v),\left(z^{\prime}, v^{\prime}\right)\right]=\left(\omega\left(v, v^{\prime}\right), 0\right)$, and

$$
\mathfrak{c s p}_{2 n}(\mathbb{R}):=\mathfrak{s p}_{2 n}(\mathbb{R}) \oplus \mathbb{R} \mathbf{1}
$$

is the conformal symplectic Lie algebra of $\left(\mathbb{R}^{2 n}, \omega\right)$. The hyperplane ideal

$$
\mathfrak{j}:=\mathfrak{h e i s}\left(\mathbb{R}^{2 n}, \omega\right) \rtimes \mathfrak{s p}_{2 n}(\mathbb{R})
$$

(the Jacobi algebra) contains a pointed generating invariant cone $C$, corresponding to the nonnegative polynomials of degree $\leq 2$ on $\mathbb{R}^{2 n}$. The involution $\tau(q, p)=(-q, p)$ is antisymplectic, and the operator $h:=\frac{1}{2}(\mathbf{1}+\tau) \in \mathfrak{c s p}_{2 n}(\mathbb{R})$ defines a 3-grading of $\mathfrak{g}$ for which the cones $C_{ \pm}$ generate $\mathfrak{g}^{ \pm 1}(h)$. The corresponding simply connected group $G$ has an irreducible unitary representation on

$$
\mathcal{H}:=L^{2}\left(\mathbb{R}_{+}^{\times}, \frac{d \lambda}{\lambda} ; L^{2}\left(\mathbb{R}^{n}\right)\right) \cong L^{2}\left(\mathbb{R}_{+}^{\times} \times \mathbb{R}^{n}, \frac{d \lambda}{\lambda} \otimes d x\right),
$$

where $\lambda$ parametrizes a family of mutually inequivalent irreducible representations of the Jacobi group on $L^{2}\left(\mathbb{R}^{n}\right)$. It would be very interesting to identify nets of standard subspaces in this representation if it is extended to an antiunitary representation of $G_{\tau}$ by

$$
(J f)(\lambda, x):=\overline{f(\lambda,-x)} .
$$

\subsection{Relation to causal structures}

In Section 5 we constructed nets of cyclic subspaces on three levels:

- On the group level, where the invariant cone $C \subseteq \mathfrak{g}$ defines a biinvariant causal structure on the group manifold $G$ and the semigroup $S^{0}=\exp \left(C_{+}^{0}\right)\left(G^{\tau_{G}}\right)_{0} \exp \left(C_{-}^{0}\right)$ plays the role of a wedge domain.

- On the symmetric space $M=G / G^{\tau_{G}}$, whose tangent space in the origin is $\mathfrak{q}=\mathfrak{g}^{1} \oplus \mathfrak{g}^{-1}$, in which $C_{\mathfrak{q}}:=C \cap \mathfrak{q}=C_{+}-C_{-}$is a pointed generating $\operatorname{Ad}\left(G^{\tau_{G}}\right)$-invariant cone that defines an invariant causal structure on $M$. Here the image of $S$ in $M$, which is the set $\operatorname{Exp}\left(C_{+}+C_{-}\right)$, where $\operatorname{Exp}: \mathfrak{q} \rightarrow M$ is the exponential function of the symmetric space $M$, plays the role of a wedge domain in $M$. Actually it also is the causal future of the base point for another causal structure on $M$ corresponding to the cone $C_{+}+C_{-}$. Note that the first causal structure turns $M$ into a compactly causal symmetric space in the sense of [HÓ97] and the second one into a non-compactly causal symmetric space.

- For the Jordan space-time $M=\widetilde{G} / \widetilde{P}^{-}$, the tangent space in the base point is $\mathfrak{g}^{1}$, which we considered also as a euclidean Jordan algebra $E$, and the causal structure is given by the cone $C_{+}=\overline{E_{+}}$. Here the image of $S$ coincides with the image of $\exp \left(C_{+}\right)$in $M$. It plays the role of a wedge domain and the future of the base point for the causal structure at the same time.

These observations show that, to proceed beyond the class of spaces considered here, we need a better theory of wedge domains in ordered homogeneous spaces. Some first steps in this general program will be carried out in [NÓ20] for the classes of compactly causal and non-compactly causal symmetric spaces, such as de Sitter spaces.

\section{A Distribution vectors for unitary representations}

In this appendix we collect some material on distribution vectors of unitary representations that we use in this paper. 
Definition A.1. Let $G$ be a Lie group. We fix a left invariant Haar measure $\mu_{G}$ on $G$ and we often write $d g$ for $d \mu_{G}(g)$. This measure defines on $L^{1}(G):=L^{1}\left(G, \mu_{G}\right)$ the structure of a Banach-*-algebra by the convolution product and

$$
(\varphi * \psi)(x)=\int_{G} \varphi(g) \psi\left(g^{-1} x\right) d \mu_{G}(g), \quad \text { and } \quad \varphi^{*}(g)=\overline{\varphi\left(g^{-1}\right)} \Delta_{G}(g)^{-1}
$$

is the involution, where $\Delta_{G}: G \rightarrow \mathbb{R}_{+}$is the modular function determined by

$$
\begin{aligned}
\int_{G} \varphi(y) d \mu_{G}(y) & =\int_{G} \varphi\left(y^{-1}\right) \Delta_{G}(y)^{-1} d \mu_{G}(y) \quad \text { and } \\
\Delta_{G}(x) \int_{G} \varphi(y x) d \mu_{G}(y) & =\int_{G} \varphi(y) d \mu_{G}(y) \quad \text { for } \quad \varphi \in C_{c}(G) .
\end{aligned}
$$

We put

$$
\varphi^{\vee}(g)=\varphi\left(g^{-1}\right) \cdot \Delta_{G}(g)^{-1} \quad \text { so that } \quad \int_{G} \varphi(g) d \mu_{G}(g)=\int_{G} \varphi^{\vee}(g) d \mu_{G}(g) .
$$

The formulas above show that we have two isometric actions of $G$ on $L^{1}(G)$, given by

$$
\left(\lambda_{g} f\right)(x)=f\left(g^{-1} x\right) \quad \text { and } \quad\left(\rho_{g} f\right)(x)=f(x g) \Delta_{G}(g) .
$$

Note that

$$
\left(\lambda_{g} f\right)^{*}=\rho_{g} f^{*} \quad \text { and } \quad\left(\lambda_{g} f\right)^{\vee}=\rho_{g} f^{\vee} .
$$

Now let $(U, \mathcal{H})$ be a continuous unitary representation of the Lie group $G$, i.e., a homomorphism $U: G \rightarrow \mathrm{U}(\mathcal{H}), g \mapsto U(g)$ such that, for each $\eta \in \mathcal{H}$, the orbit map $U^{\eta}(g)=U(g) \eta$ is continuous. For $\varphi \in L^{1}(G)$ the operator-valued integral

$$
U(\varphi):=\int_{G} \varphi(g) U(g) d g
$$

exists and is uniquely determined by

$$
\langle\eta, U(\varphi) \zeta\rangle=\int_{G} \varphi(g)\langle\eta, U(g) \zeta\rangle d g \quad \text { for } \quad \eta, \zeta \in \mathcal{H} .
$$

Then $\|U(\varphi)\| \leq\|\varphi\|_{1}$ and the so-obtained continuous linear map $L^{1}(G) \rightarrow B(\mathcal{H})$ is a representation of the Banach-*-algebra $L^{1}(G)$, i.e., $U(\varphi * \psi)=U(\varphi) U(\psi)$ and $U\left(\varphi^{*}\right)=U(\varphi)^{*}$. We also note that

$$
U(g) U(\varphi)=U\left(\lambda_{g} \varphi\right) \quad \text { and } \quad U(\varphi) U(g)=U\left(\rho_{g}^{-1} \varphi\right) \quad \text { for } \quad g \in G, \varphi \in L^{1}(G) .
$$

For $\varphi_{g}(x):=\varphi(x g)$, we then have $\varphi_{g}=\Delta_{G}(g)^{-1} \rho_{g} \varphi$ by (77), and thus by (80)

$$
U\left(\varphi_{g}\right)=\Delta_{G}(g)^{-1} U(\varphi) U\left(g^{-1}\right) \quad \text { for } \quad g \in G .
$$

\section{A.1 The topology on $\mathcal{H}^{\infty}$ and the space $\mathcal{H}^{-\infty}$}

A smooth vector is an element $\eta \in \mathcal{H}$ for which the orbit map $U^{\eta}: G \rightarrow \mathcal{H}, g \mapsto U(g) \eta$ is smooth. We write $\mathcal{H}^{\infty}$ for the space of smooth vectors. It carries the derived representation $\mathrm{d} U$ of the Lie algebra $\mathfrak{g}$ given by

$$
\mathrm{d} U(x) \eta=\lim _{t \rightarrow 0} \frac{U(\exp t x) \eta-\eta}{t} .
$$


We extend this representation to a homomorphism $\mathrm{d} U: \mathcal{U}(\mathfrak{g}) \rightarrow \operatorname{End}\left(\mathcal{H}^{\infty}\right)$, where $\mathcal{U}(\mathfrak{g})$ is the complex enveloping algebra of $\mathfrak{g}$. This algebra carries an involution $D \mapsto D^{*}$ determined uniquely $x^{*}=-x$ for $x \in \mathfrak{g}$. For $D \in \mathcal{U}(\mathfrak{g})$, we obtain a seminorm on $\mathcal{H}^{\infty}$ by

$$
p_{D}(\eta)=\|\mathrm{d} U(D) \eta\| \quad \text { for } \quad \eta \in \mathcal{H}^{\infty} .
$$

These seminorms define a topology on $\mathcal{H}^{\infty}$ which turn the injection

$$
\eta: \mathcal{H}^{\infty} \rightarrow \mathcal{H}^{\mathcal{U}\left(\mathfrak{g}_{\mathbb{C}}\right)}, \quad \xi \mapsto(\mathrm{d} U(D) \xi)_{D \in \mathcal{U}\left(\mathfrak{g}_{\mathbb{C}}\right)}
$$

into a topological embedding, where the right hand side carries the product topology (cf. Mag92, 3.19]). It turns $\mathcal{H}^{\infty}$ into a complete locally convex space for which the linear operators $\mathrm{d} U(D), D \in \mathcal{U}(\mathfrak{g})$, are continuous. Since $\mathcal{U}(\mathfrak{g})$ has a countable basis, countably many such seminorms already determine the topology, so that $\mathcal{H}^{\infty}$ is metrizable. As it is also complete, it is a Fréchet space. We also observe that the inclusion $\mathcal{H}^{\infty} \hookrightarrow \mathcal{H}$ is continuous.

The space $\mathcal{H}^{\infty}$ of smooth vectors is $G$-invariant and we denote the corresponding representation by $U^{\infty}$. We have the intertwining relation

$$
\mathrm{d} U(\operatorname{Ad}(g) x)=U(g) \mathrm{d} U(x) U(g)^{-1} \quad \text { for } \quad g \in G, x \in \mathfrak{g} .
$$

If $\varphi \in C_{c}^{\infty}(G)$ and $\xi \in \mathcal{H}$, then $U(\varphi) \xi \in \mathcal{H}^{\infty}$ and differentiation under the integral sign shows that

$$
\mathrm{d} U(x) U(\varphi) \xi:=U\left(-x^{R} \varphi\right) \xi, \quad \text { where } \quad\left(x^{R} \varphi\right)(g)=\left.\frac{d}{d t}\right|_{t=0} \varphi((\exp t x) g) .
$$

A sequence $\left(\varphi_{n}\right)_{n \in \mathbb{N}}$ in $C_{c}^{\infty}(G)$ is called a $\delta$-sequence if $\int_{G} \varphi_{n}(g) d g=1$ for every $n \in \mathbb{N}$ and, for every e-neighborhood $U \subseteq G$, we have $\operatorname{supp}\left(\varphi_{n}\right) \subseteq U$ if $n$ is sufficiently large. If $\left(\varphi_{n}\right)_{n \in \mathbb{N}}$ is a $\delta$-sequence, then $U\left(\varphi_{n}\right) \xi \rightarrow \xi$, so that $\mathcal{H}^{\infty}$ is dense in $\mathcal{H}$.

We write $\mathcal{H}^{-\infty}$ for the space of continuous anti-linear functionals on $\mathcal{H}^{\infty}$. Its elements are called distribution vectors. The group $G, \mathcal{U}(\mathfrak{g})$ and $C_{c}^{\infty}(G)$ act on $\eta \in \mathcal{H}^{-\infty}$ by

- $\left(U^{-\infty}(g) \eta\right)(\xi):=\eta\left(U\left(g^{-1}\right) \xi\right), g \in G, \xi \in \mathcal{H}^{\infty}$. If $U: G \rightarrow \mathrm{AU}(\mathcal{H})$ is an antiunitary representation and $U(g)$ is antiunitary, then we have to modify this definition slightly by $\left(U^{-\infty}(g) \eta\right)(\xi):=\overline{\eta\left(U\left(g^{-1}\right) \xi\right)}$.

- $\left(\mathrm{d} U^{-\infty}(D) \eta\right)(\xi):=\eta\left(\mathrm{d} U\left(D^{*}\right) \xi\right), D \in \mathcal{U}(\mathfrak{g}), \xi \in \mathcal{H}^{\infty}$.

- $U^{-\infty}(\varphi) \eta=\eta \circ U^{\infty}\left(\varphi^{*}\right), \varphi \in C_{c}^{\infty}(G)$.

We have natural $G$-equivariant linear embeddings

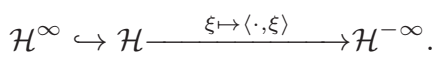

It is an important feature of (85) that the representation of $\mathcal{U}(\mathfrak{g})$ on $\mathcal{H}^{-\infty}$ provides an embedding of the whole Hilbert space $\mathcal{H}$ into a larger space on which the Lie algebra acts. The following lemma shows that, $\mathcal{H}^{\infty}$ is the maximal $\mathfrak{g}$-invariant subspace of $\mathcal{H} \subseteq \mathcal{H}^{-\infty}$ and that the subspace $\mathcal{H}$ generates $\mathcal{H}^{-\infty}$ as a $\mathfrak{g}$-module.

Lemma A.2. The following assertions hold:

(a) $\mathcal{H}^{\infty}=\left\{\xi \in \mathcal{H} \subseteq \mathcal{H}^{-\infty}:(\forall D \in \mathcal{U}(\mathfrak{g})) \mathrm{d} U^{-\infty}(D) \xi \in \mathcal{H}\right\}$.

(b) $\mathcal{H}^{-\infty}=\operatorname{span}\left(\mathrm{d} U^{-\infty}(\mathcal{U}(\mathfrak{g})) \mathcal{H}\right)$.

Proof. (a) This follows by combining Oeh18, Prop. A.1], asserting that

$$
\mathcal{D}(\partial U(x))=\left\{\xi \in \mathcal{H}: \mathrm{d} U^{-\infty}(x) \xi \in \mathcal{H}\right\},
$$

with the fact that

$$
\mathcal{H}^{\infty}=\bigcap\left\{\mathcal{D}\left(\partial U\left(x_{1}\right) \cdots \partial U\left(x_{n}\right)\right): n \in \mathbb{N}, x_{1}, \ldots, x_{n} \in \mathfrak{g}\right\}
$$


( Ne10, Lemma 3.4]).

(b) Let $\eta \in \mathcal{H}^{-\infty}$ and consider $\mathcal{H}^{\infty}$ as a subspace of the topological product $\mathcal{H}^{\mathcal{U}(\mathfrak{g})}$. By the Hahn-Banach extension theorem, $\eta$ extends to a continuous antilinear functional $\widetilde{\eta}$ on $\mathcal{H}^{\mathcal{U}(\mathfrak{g})}$. Since the dual of a direct product is the direct sum of the dual spaces, there exist $D_{1}, \ldots, D_{n} \in \mathcal{U}(\mathfrak{g})$ and $\xi_{1}, \ldots, \xi_{n} \in \mathcal{H}$, such that

$$
\eta(\xi)=\sum_{j=1}^{n}\left\langle\mathrm{~d} U\left(D_{j}\right) \xi, \xi_{j}\right\rangle=\sum_{j=1}^{n}\left\langle\xi, \mathrm{d} U^{-\infty}\left(D_{j}^{*}\right) \xi_{j}\right\rangle \quad \text { for } \quad \xi \in \mathcal{H}^{\infty}
$$

which means that $\eta=\sum_{j=1}^{n} \mathrm{~d} U^{-\infty}\left(D_{j}^{*}\right) \xi_{j}$.

For each $\varphi \in C_{c}^{\infty}(G)$, the map $U(\varphi): \mathcal{H} \rightarrow \mathcal{H}^{\infty}$ is continuous, so that its adjoint defines a weak-*-continuous maps $U^{-\infty}\left(\varphi^{*}\right): \mathcal{H}^{-\infty} \rightarrow \mathcal{H}$. We actually have $U^{-\infty}(\varphi) \mathcal{H}^{-\infty} \subseteq \mathcal{H}^{\infty}$ as a consequence of the Dixmier-Malliavin Theorem [DM78, Thm. 3.1], which asserts that every $\varphi \in C_{c}^{\infty}(G)$ factors as $\varphi=\varphi_{1} * \varphi_{2}$ with $\varphi_{j} \in C_{c}^{\infty}(G)$

\section{A.2 Equivariant embeddings into distributions on $G$}

Let $G$ be a Lie group and $(U, \mathcal{H})$ be a unitary representation of $G$. We call a distribution vector $\eta \in \mathcal{H}^{-\infty}$ cyclic if $U^{-\infty}\left(C_{c}^{\infty}(G)\right) \eta$ is dense in $\mathcal{H}$. Then [NÓ18, Prop. 7.1.6] yields a G-equivariant injection

$$
j_{\eta}: \mathcal{H}^{-\infty} \rightarrow C^{-\infty}(G), \quad j_{\eta}(\alpha)(\varphi):=\alpha\left(U^{-\infty}(\varphi) \eta\right) \quad \text { for } \quad \varphi \in C_{c}^{\infty}(G)
$$

In particular,

is positive definite and

$$
D:=j_{\eta}(\eta) \in C^{-\infty}(G)
$$

$$
\mathcal{H}_{D}:=j_{\eta}(\mathcal{H}) \subseteq C^{-\infty}(G)
$$

is a reproducing kernel Hilbert space of distributions, on which $G$ acts by left translations. For $\varphi, \psi \in C_{c}^{\infty}(G)$, we have

$$
\begin{gathered}
j_{\eta} \circ U^{-\infty}(g)=\lambda_{g} \circ j_{\eta} \quad \text { for } \quad g \in G, \\
j_{\eta}\left(U^{-\infty}(\varphi) \alpha\right)=\varphi * j_{\eta}(\alpha) \quad \text { for } \quad \varphi \in C_{c}^{\infty}(G),
\end{gathered}
$$

and

$$
\left\langle U^{-\infty}(\varphi) \eta, U^{-\infty}(\psi) \eta\right\rangle_{\mathcal{H}}=\langle\varphi * D, \psi * D\rangle_{\mathcal{H}_{D}}=D\left(\psi^{*} * \varphi\right) .
$$

Remark A.3. To obtain embeddings into $C^{-\infty}(G)$ that are equivariant with respect to the action by right translations, we first extend the involution $\vee$ from (76) to distributions by

$$
D^{\vee}(\varphi):=D\left(\varphi^{\vee}\right)
$$

to obtain by duality and (78)

$$
\left(\lambda_{g} D\right)^{\vee}=\rho_{g} D^{\vee} \quad \text { for } \quad g \in G .
$$

Therefore the map

$$
j_{\eta}^{\vee}: \mathcal{H}^{-\infty} \rightarrow C^{-\infty}(G), \quad j_{\eta}^{\vee}(\alpha)(\varphi):=\alpha\left(U^{-\infty}\left(\varphi^{\vee}\right) \eta\right)
$$

is equivariant with respect to the action of $G$ on $C^{-\infty}(G)$ by right translations. 


\section{References}

[Ar63] Araki, H., A lattice of von Neumann algebras associated with the quantum theory of a free Bose field, J. Math. Phys. 4 (1963), 1343-1362

[Ar64] Araki, H., von Neumann algebras of local observables for free scalar field, J. Mathematical Phys. 5 (1964), 1-13

[AW63] Araki, H., and E. J. Woods, Representations of the canonical commutation relations describing a nonrelativistic infinite free Bose gas, J. Math. Phys. 4 (1963), 637-662

[AW68] Araki, H., and E. J. Woods, A classification of factors, Publ. RIMS, Kyoto Univ. Ser. A 3 (1968), 51-130

[BJL02] Baumgärtel, H., Jurke, M., and F. Lledo, Twisted duality of the CAR-algebra, J. Math. Physics 43:8 (2002), 4158-4179

[Bo92] Borchers, H.-J., The CPT-Theorem in two-dimensional theories of local observables, Comm. Math. Phys. 143 (1992), 315-332

[BR87] Bratteli, O., and D. W. Robinson, "Operator Algebras and Quantum Statistical Mechanics I," 2nd ed., Texts and Monographs in Physics, Springer-Verlag, 1987

[BM96] Bros, J., and U. Moschella, Two-point functions and quantum fields in de Sitter universe, Rev. Math. Phys. 8 (1996), 327-391

[BGL93] Brunetti, R., Guido, D., and R. Longo, Modular structure and duality in conformal quantum field theory, Comm. Math. Phys. 156 (1993), 210-219

[BGL02] Brunetti, R., Guido, D., and R. Longo, Modular localization and Wigner particles, Rev. Math. Phys. 14 (2002), 759-785

[BDFS00] Buchholz, D., Dreyer, O., Florig, M., and S. J. Summers, Geometric modular action and spacetime symmetry groups, Rev. Math. Phys. 12:4 (2000), 475-560

[BLS11] Buchholz, D., Lechner, G., and S. J. Summers, Warped convolutions, Rieffel deformations and the construction of quantum field theories, Comm. Math. Phys. 304:1 (2011), 95-123

[DM78] Dixmier, J., and P. Malliavin, Factorisations de fonctions et de vecteurs indéfiniment différentiables, Bull. Soc. math., 2e série 102 (1978), 305-330

[EO73] Eckmann, J.-P., and K. Osterwalder, An application of Tomita's theory of modular Hilbert algebras: duality for free Bose fields, J. Funct. Analysis 13:1 (1973), 1-12

[EHW83] Enright, T.J., R. Howe, and N. Wallach, A classification of unitary highest weight modules, in "Representation Theory of Reductive Groups," Progress in Math. 40, Birkhäuser Verlag, 1983, 97-143

[FK94] Faraut, J., and A. Koranyi, "Analysis on Symmetric Cones," Oxford Math. Monographs, Oxford University Press, 1994

[GN] Glöckner, H., and K.-H. Neeb, "Infinite Dimensional Lie Groups, Vol. I, Basic Theory and Main Examples," book in preparation

[Gu93] Günaydin, M., Generalized conformal and superconformal group actions and Jordan algebras, Modern Phys. Letters A 8:15 (1993), 1407-1416

[Ha96] Haag, R., "Local Quantum Physics. Fields, Particles, Algebras," Second edition, Texts and Monographs in Physics, Springer-Verlag, Berlin, 1996

[HN01] Hilgert, J., and K.-H. Neeb, Vector-valued Riesz distributions on euclidian Jordan algebras, J. Geom. Analysis 11:1 (2001), 43-75

[HN12] Hilgert, J., and K.-H. Neeb, "Structure and Geometry of Lie Groups," Springer Monographs in Mathematics, Springer, New York, 2012 
[HÓ97] Hilgert, J., and G. Ólafsson, Causal Symmetric Spaces, Geometry and Harmonic Analysis, Perspectives in Mathematics 18, Academic Press, 1997

[Iw59] Iwahori, N., On real irreducible representations of Lie algebra, Nagoya Math. J. 14 (1959), 59-83

[Le15] Lechner, G., Algebraic Constructive Quantum Field Theory: Integrable Models and Deformation Techniques, in "Advances in Algebraic Quantum Field Theory," Eds. R. Brunetti et al; Math. Phys. Stud., Springer, 2015, 397-449, arXiv:math.ph:1503.03822

[LL15] Lechner, G., and R. Longo, Localization in Nets of Standard Spaces, Comm. Math. Phys. 336:1 (2015), 27-61

[Lo08] Longo, R., Real Hilbert subspaces, modular theory, $\mathrm{SL}(2, \mathbb{R})$ and CFT in "Von Neumann Algebras in Sibiu", 33-91, Theta Ser. Adv. Math. 10, Theta, Bucharest, 2008

[Lo69] Loos, O., "Symmetric Spaces I: General Theory," W. A. Benjamin, Inc., New York, Amsterdam, 1969

[MdR07] Mack, G., and M. de Riese, Simple space-time symmetries: generalizing conformal field theory, J. Math. Phys. 48:5 (2007), 052304, 21 pp.

[Mag92] Magyar, M., "Continuous Linear Representations," North-Holland, Mathematical Studies 168, 1992

[MN20] Morinelli, V., and K.-H. Neeb, Covariant homogeneous nets of standard subspaces, in preparation

[Ne98] Neeb, K.-H., Operator valued positive definite kernels on tubes, Monatshefte für Math. 126 (1998), 125-160

[Ne99] Neeb, K.-H., "Holomorphy and Convexity in Lie Theory," Expositions in Mathematics 28, de Gruyter Verlag, Berlin, 1999

[Ne10] Neeb, K.-H., On differentiable vectors for representations of infinite dimensional Lie groups, J. Funct. Anal. 259 (2010), 2814-2855

[Ne13] Neeb, K.-H., Holomorphic realization of unitary representations of Banach-Lie groups, in "Lie Groups: Structure, Actions, and Representations-In Honor of Joseph A. Wolf on the Occasion of his 75th Birthday," Huckleberry, A., Penkov, I., Zuckerman, G. (Eds.), Progress in Mathematics 306, 2013; 185-223

[Ne19] Neeb, K.-H., Finite dimensional semigroups of unitary endomorphisms of standard subspaces, arXiv:math.OA.1902.02266

[Ne19b] Neeb, K.-H., Semigroups in 3-graded Lie groups, arXiv:math.OA.1912.13367

[NÓ17] Neeb, K.-H., and G. Ólafsson, Antiunitary representations and modular theory, in "50th Sophus Lie Seminar", Eds. K. Grabowska et al, Banach Center Publications 113; pp. 291-362; arXiv:math-RT:1704.01336

[NÓ18] Neeb, K.-H., and G. Ólafsson, "Reflection Positivity. A Representation Theoretic Perspective," Springer Briefs in Mathematical Physics 32, 2018

[NÓ20] Neeb, K.-H., and G. Ólafsson, Standard subspaces of Hilbert spaces of distributions on noncompactly causal symmetric spaces, in preparation

[NÓO20] Neeb, K.-H., G. Ólafsson, and B. Ørsted, Standard subspaces of Hilbert spaces of holomorphic functions on tube domains, in preparation

[Oeh18] Oeh, D., Analytic extensions of representations of $\star$-semigroups without polar decomposition, Internat. Math. Res. Notices, to appear; arXiv:math-RT:1812.10751 
[Ó91] Ólafsson, G., Symmetric spaces of Hermitian type, Differential Geom. Appl. 1 (1991), 195-233

[On20] Onishchik, A.L., "Lectures on Real Semisimple Lie Algebras and Their Representations," ESI Lectures in Mathematics and Physics, European Math. Soc. Publishing House, 2020

[PEGW19] Pejhan, H., Enayati, M., Gazeau, J.-P., and A. Wang, "Massive" Rarita-Schwinger fields in de Sitter space, arXiv:1909.12350v1 [gr-qc]

[Schr97] Schroer, B., Wigner representation theory of the Poincaré group, localization, statistics and the S-matrix, Nuclear Phys. B 499-3 (1997), 519-546

[Si74] Simon, B., "The $P(\Phi)_{2}$ Euclidean (Quantum) Field Theory", Princeton Univ. Press, 1974

[TS16] Thiemann, Th., and A. Stottmeister, The microlocal spectrum condition, initial value formulations and background independence, Journal of Mathematical Physics 57 (2016), 022303

[Tr67] Treves, F., "Topological Vector Spaces, Distributions, and Kernels," Academic Press, New York, 1967

[Wi93] Wiesbrock, H.-W., Half-sided modular inclusions of von Neumann algebras, Commun. Math. Phys. 157 (1993), 83-92

Department of Mathematics, Friedrich-Alexander-University of Erlangen-Nürnberg, Cauerstrasse 11, 91058 Erlangen, Germany; neeb@math.fau.de

Department of Mathematics, Louisiana State University, Baton Rouge, LA 70803, U.S.A.; olafsson@math.lsu.edu 